Organisation de coopération et de développement économiques

\title{
LA MESURE DES ACTIVITÉS \\ SCIENTIFIQUES \\ ET \\ TECHNOLOGIQUES
}

PRINCIPES DIRECTEURS PROPOSÉS

POUR LE RECEUIL

ET L'INTERPRÉTATION

DES DONNÉES SUR

L'INNOVATION TECHNOLOGIGIQUE

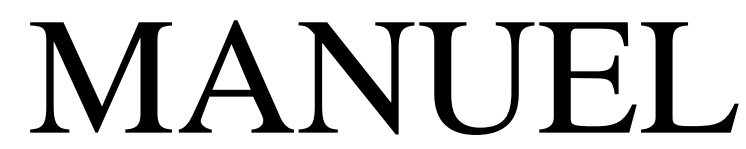

D'OSLO 
TABLE DES MATIERES

Chapitre 1 OBJECTIFS ET PORTÉE DU MANUEL ...................................................................... 5

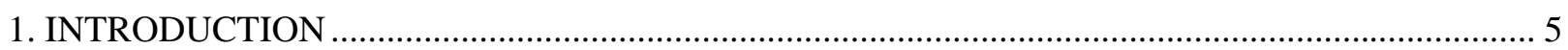

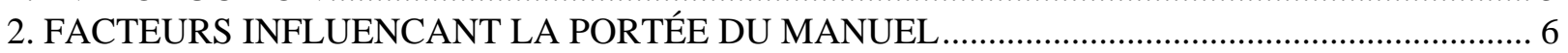

2.1 Comprendre le processus d'innovation et ses conséquences pour la politique de l'innovation ....... 6

2.2 Expérience acquise au niveau de la fourniture de données .................................................... 7

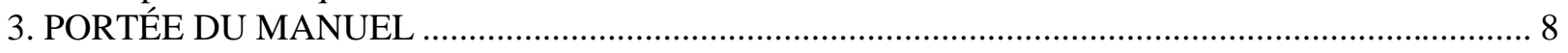

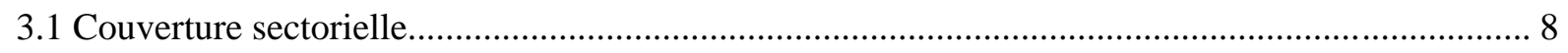

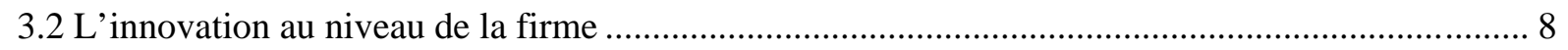

3.3 Innovation technologique de produit et de procédé - innovation TPP ........................................ 9

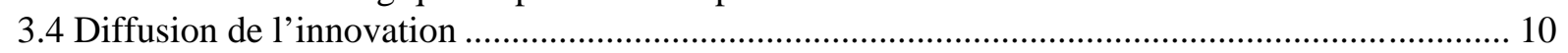

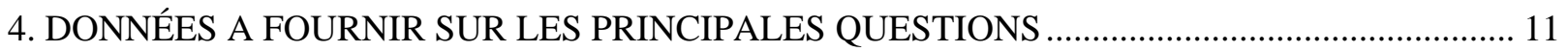

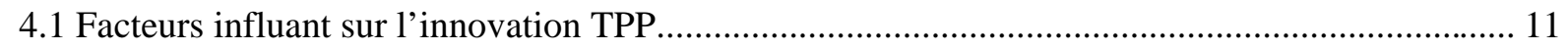

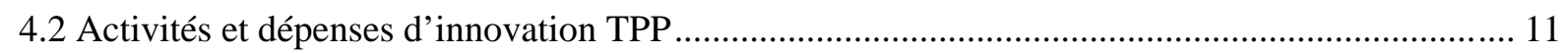

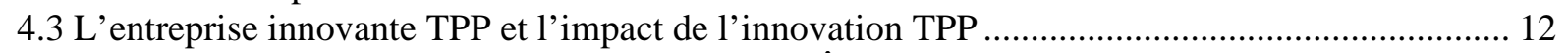

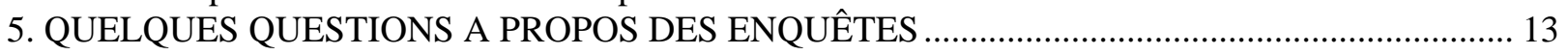

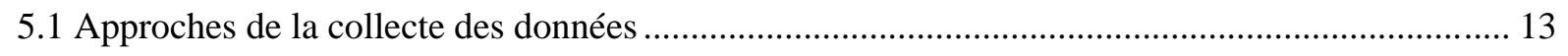

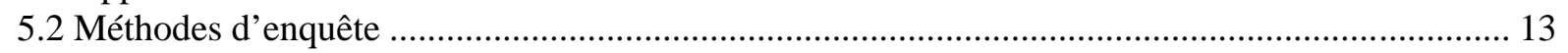

6. LES RELATIONS ENTRE LE MANUEL D'OSLO, D'AUTRES NORMES INTERNATIONALES

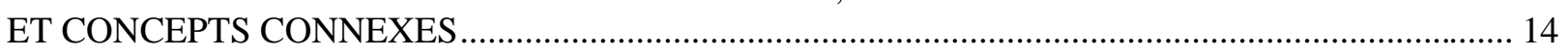

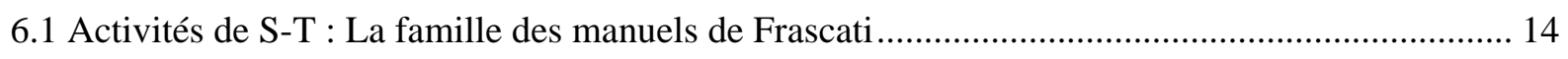

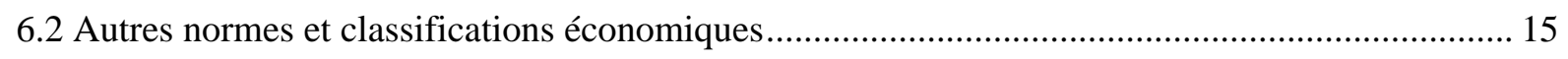

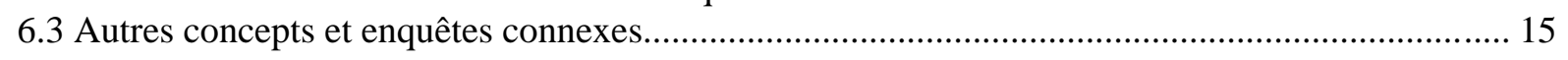

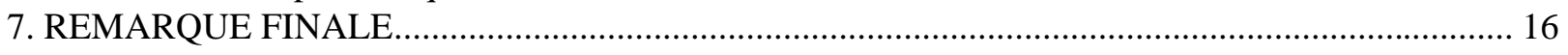

Chapitre 2 LA NÉCESSITÉ DE MESURER L'INNOVATION …................................................... 17

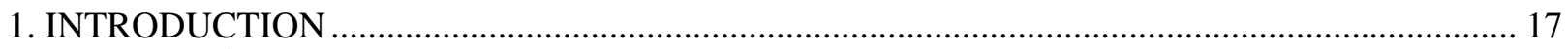

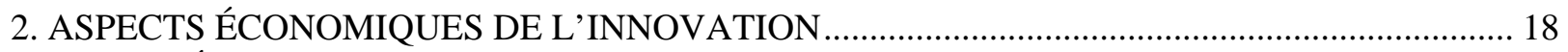

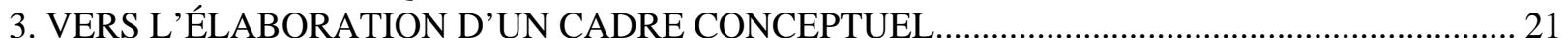

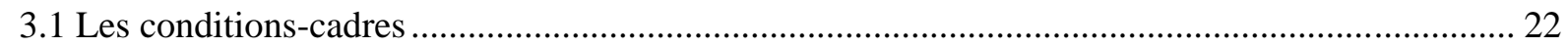

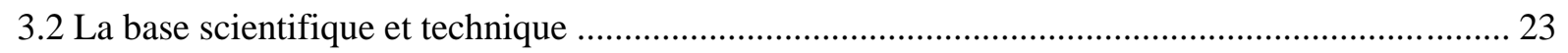

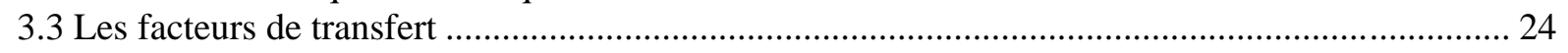

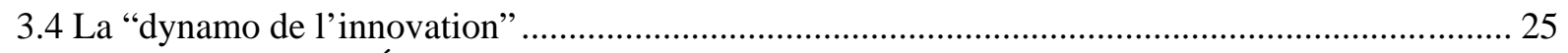

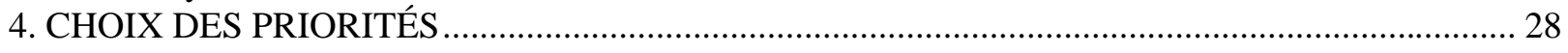

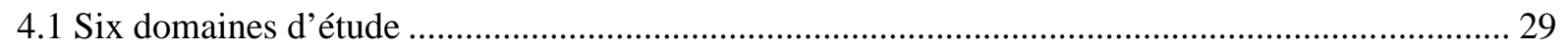

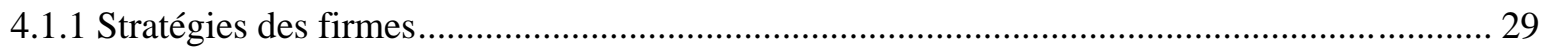

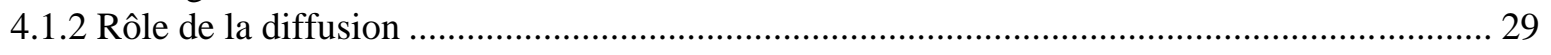

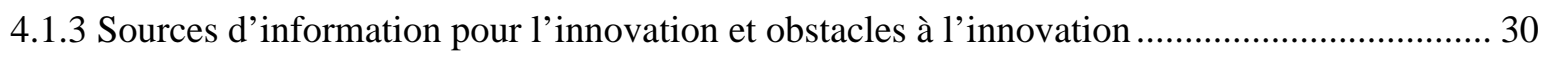

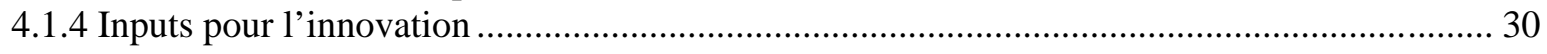

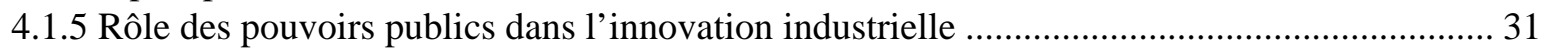


4.1.6 Outputs de l'innovation

4.2 Les moyens de mesurer l'innovation et portée de la mesure ...................................................... 32

4.2.1 "Que voulons-nous mesurer ?" : Les innovations technologiques de produit et de procédé Innovations TPP

4.2.2 "Comment voulons-nous procéder ?" : choix de l'approche pour l'enquête ............................ 33

4.2.3 "Où voulons-nous effectuer les mesures ?” : couverture sectorielle........................................ 34

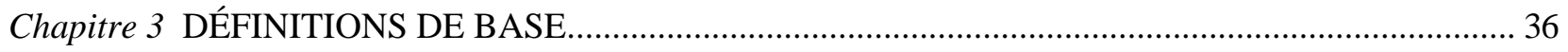

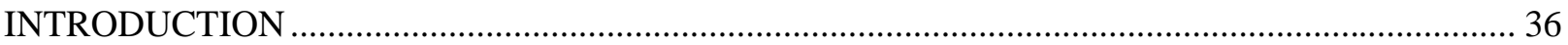

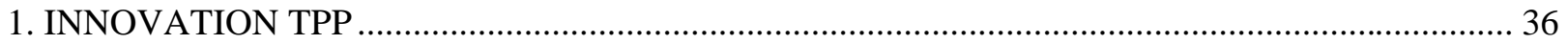

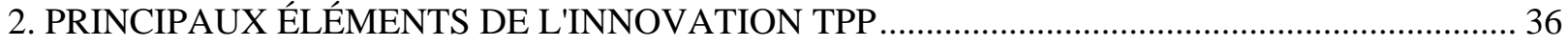

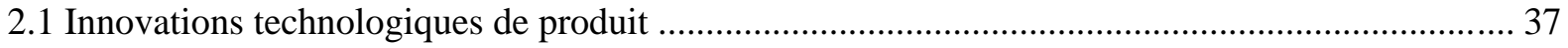

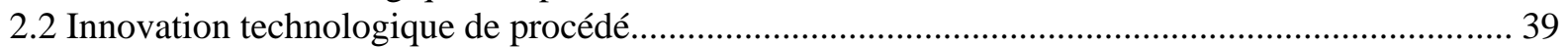

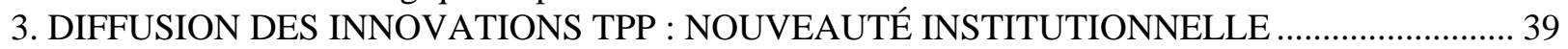

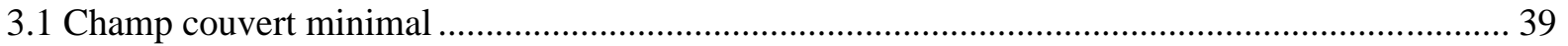

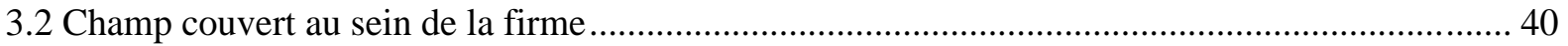

4. DISTINCTION ENTRE L'INNOVATION TPP ET LES AUTRES CHANGEMENTS AU SEIN DE

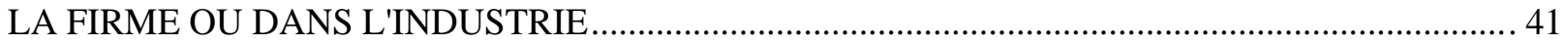

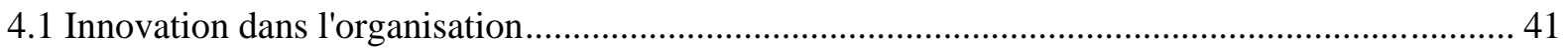

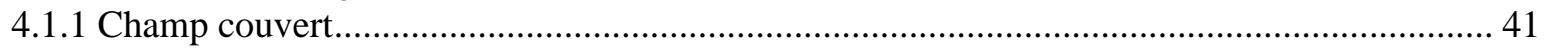

4.1.2 Cas limites : changement dans l'organisation concernant les procédés dans l'industrie

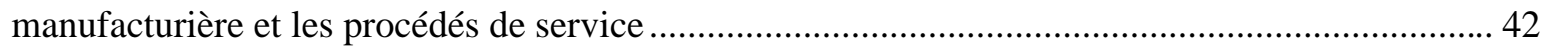

4.2 Autres changements concernant les produits et les procédés....................................................... 42

4.2.1 Exclusion de changements insignifiants ou non porteurs de nouveauté ................................. 42

(a) Cessation de l'utilisation d'un procédé ou de la commercialisation d'un produit .................... 42

(b) Simple remplacement ou accroissement de l'équipement ......................................................... 43

(c) Changements découlant uniquement de variations du prix des facteurs ................................... 43

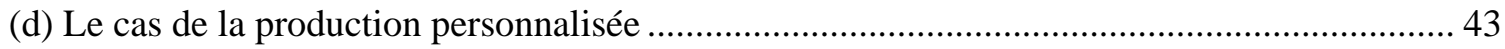

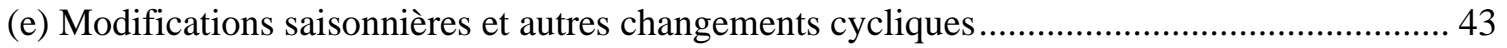

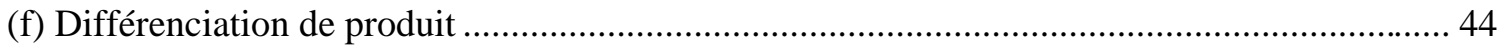

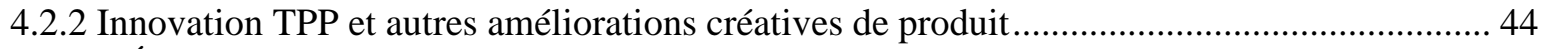

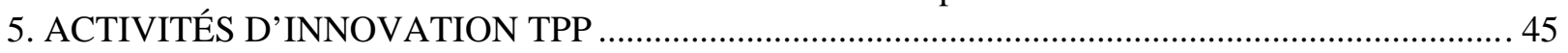

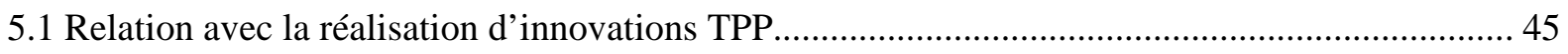

5.2 Les composantes et la couverture des activités d'innovation TPP ............................................. 45

5.2.1 Acquisition et production de connaissances pertinentes qui sont nouvelles pour la firme..... 46

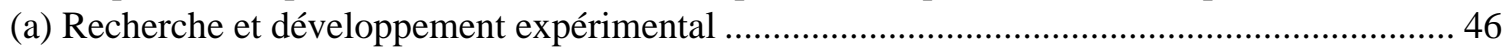

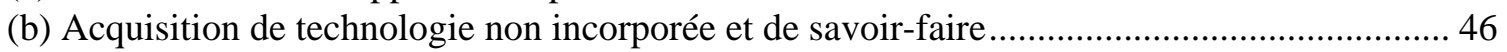

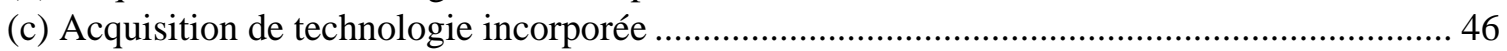

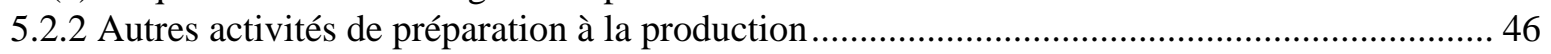

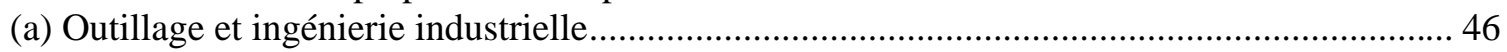

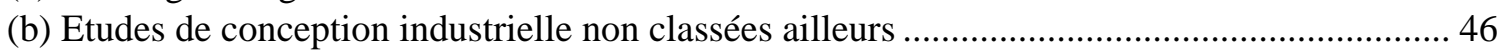

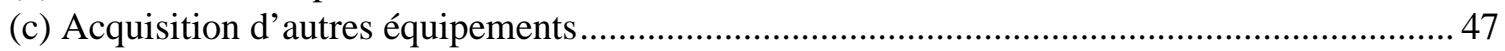

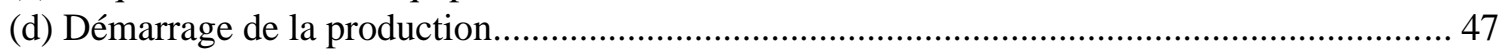

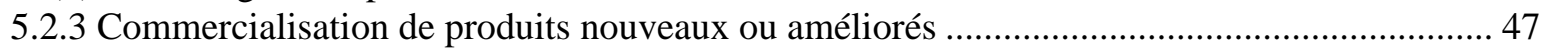

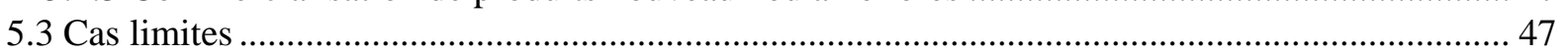

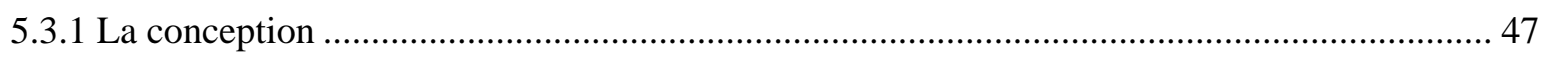

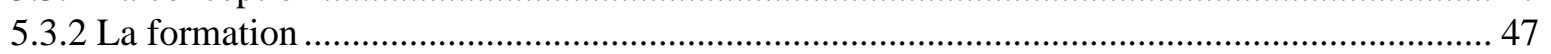

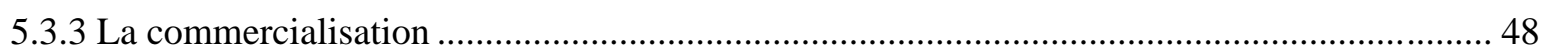

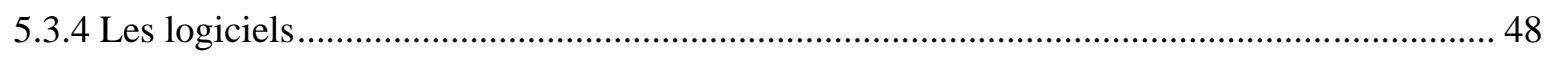




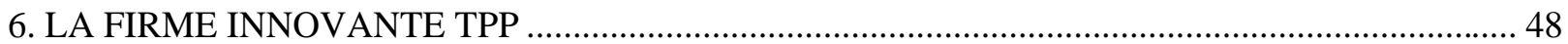

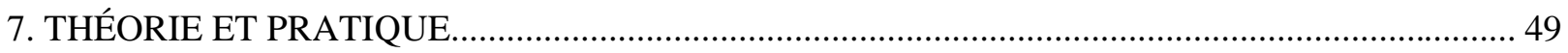

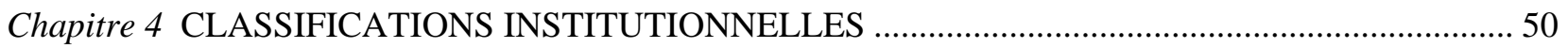

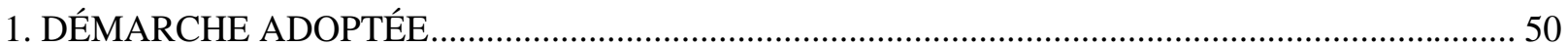

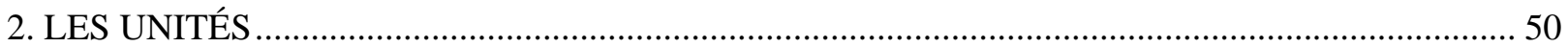

3. CLASSIFICATION SELON L'ACTIVITÉ ÉCONOMIQUE PRINCIPALE .................................... 51

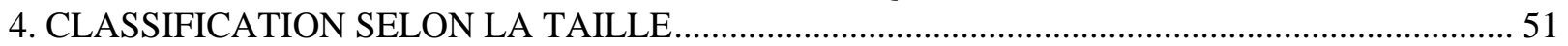

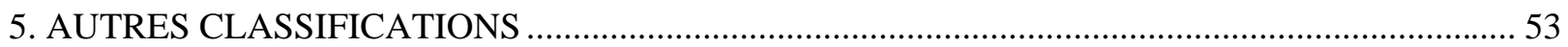

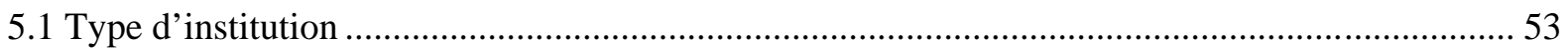

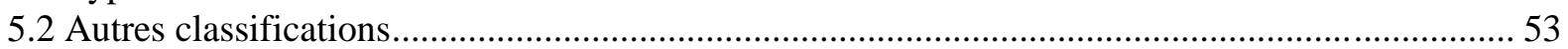

Chapitre 5 MESURE DE CERTAINS ASPECTS DU PROCESSUS D'INNOVATION ........................ 55

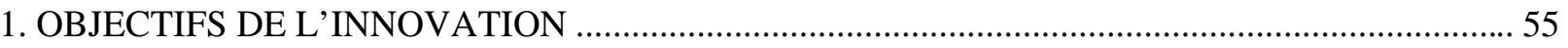

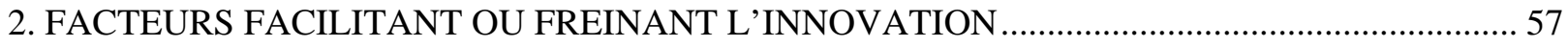

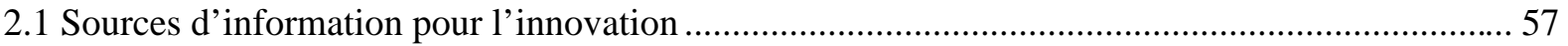

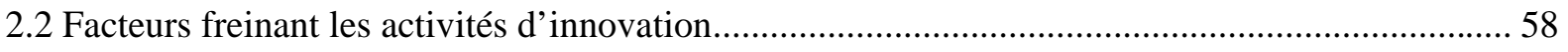

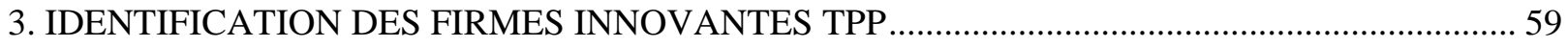

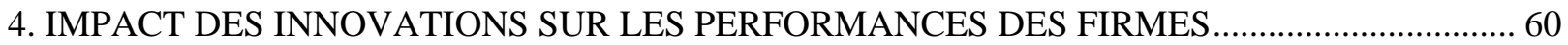

4.1 Proportion des ventes imputables aux produits technologiquement nouveaux ou améliorés......... 60

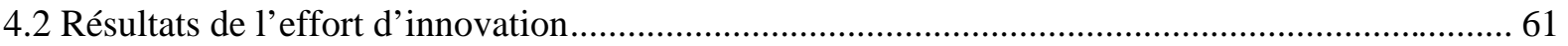

4.3 Impact de l'innovation TPP sur l'utilisation des facteurs de production ....................................... 62

4.3.1 Réductions du coût moyen imputables aux innovations technologiques de procédé .............. 62

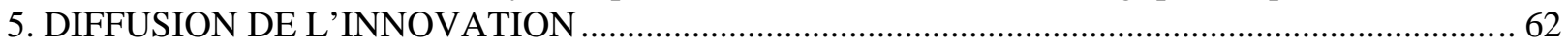

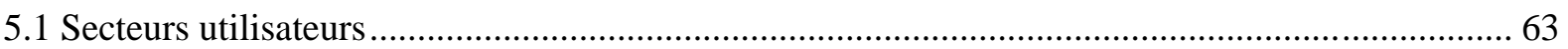

5.2 Enquêtes sur l'utilisation de technologies avancées dans les procédés de fabrication ................... 63

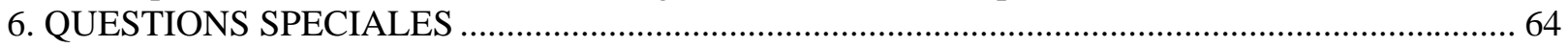

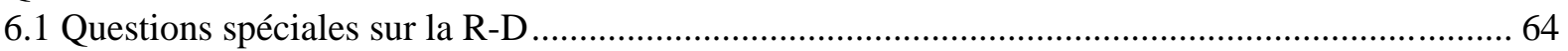

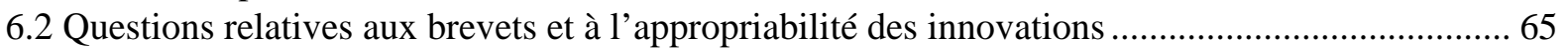

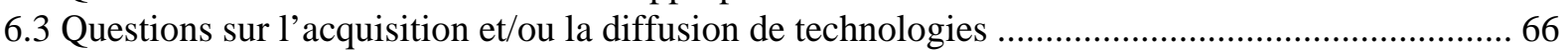

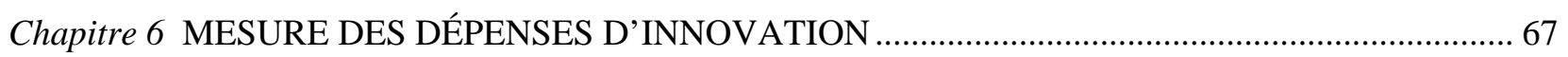

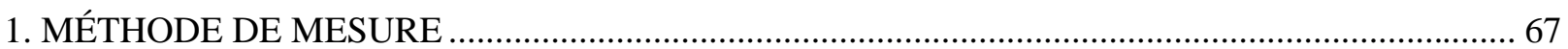

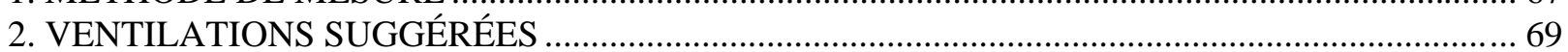

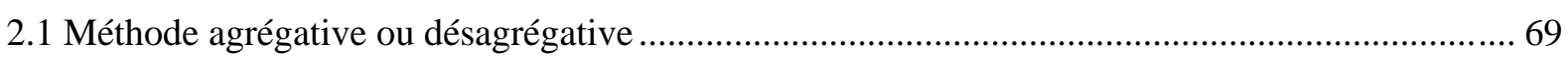

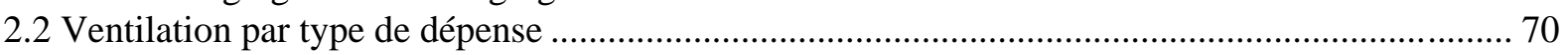

2.2.1 Relation entre les investissements immatériels et les dépenses d'innovation TPP ................. 71

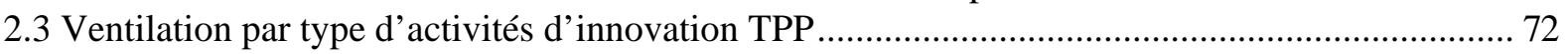

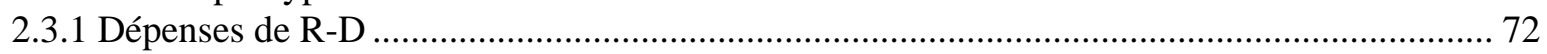

2.3.2 Dépenses encourues pour l'acquisition de technologie et de savoir-faire non incorporés ..... 73

2.3.3 Dépenses encourues pour l'acquisition de technologie incorporée ........................................ 73

2.3.4 Dépenses encourues pour l'outillage, l'ingénierie industrielle, les études de conception industrielle et le démarrage de la production (y compris les autres dépenses relatives aux installations pilotes et aux prototypes qui ne sont pas déjà incluses dans la R-D) ......................... 73

2.3.5 Dépenses de formation liées à des activités portant sur des innovations TPP ........................ 74

2.3.6 Commercialisation de produits technologiquement nouveaux ou améliorés........................... 74

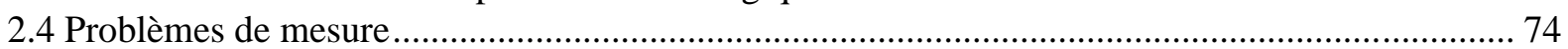

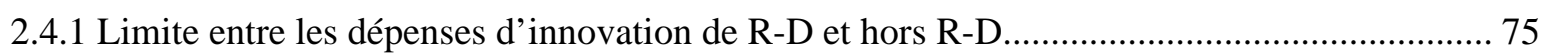

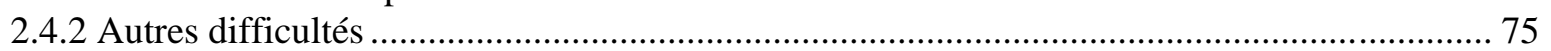

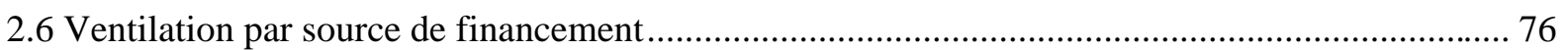




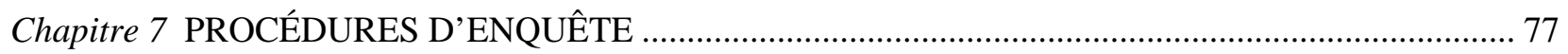

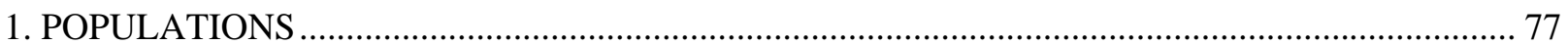

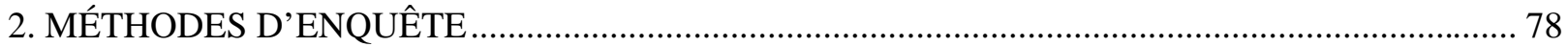

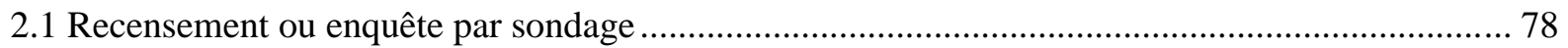

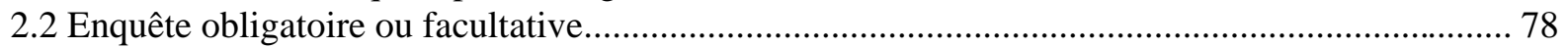

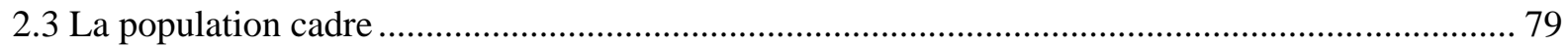

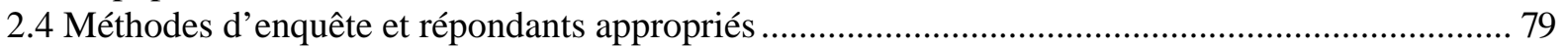

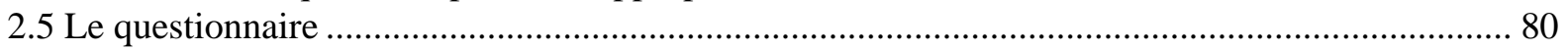

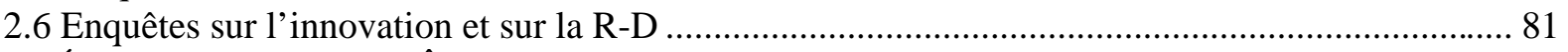

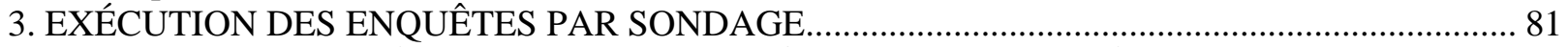

4. ESTIMATION DES RÉSULTATS - LE PROBLÈME DE LA NON-RÉPONSE ............................ 83

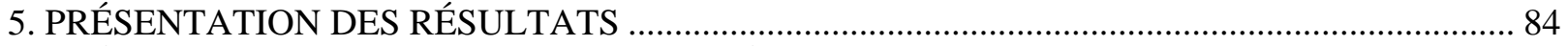

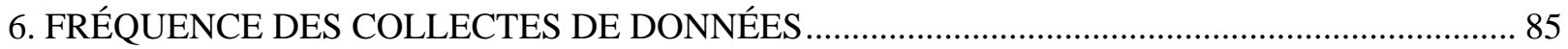

ANNEXE 1 UTILISATION DE L'APPROCHE OBJET POUR LA COLLECTE DE DONNÉES SUR

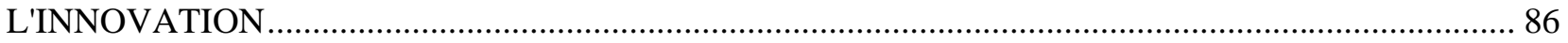

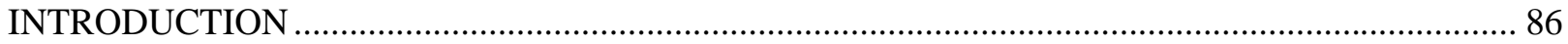

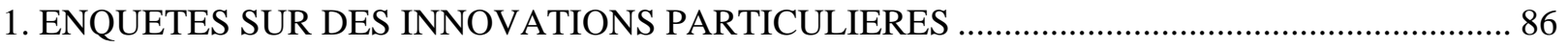

1.1. Questions abordées lors de l'utilisation de données fournies par l'approche objet......................... 86

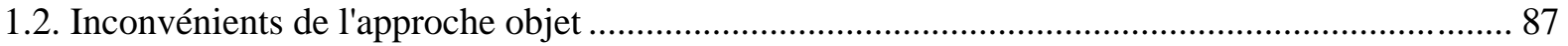

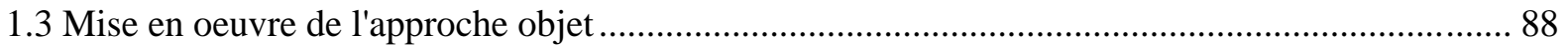

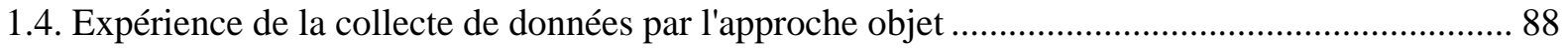

1.5. Données susceptibles d'être recueillies par l'approche objet......................................................... 89

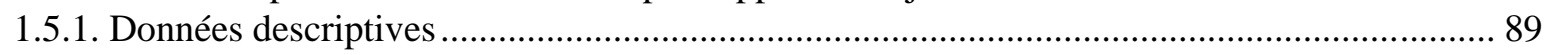

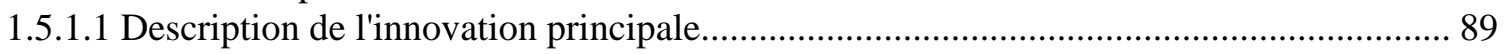

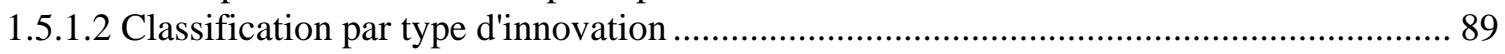

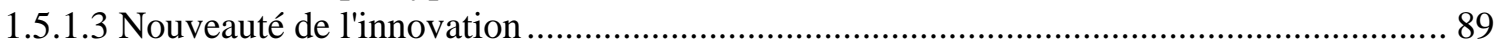

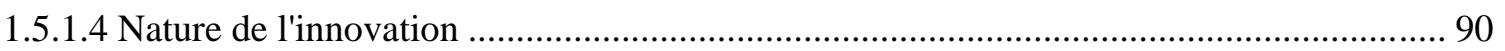

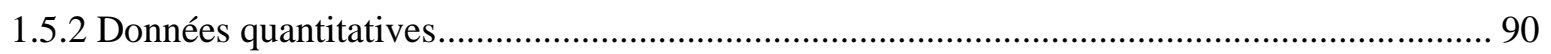

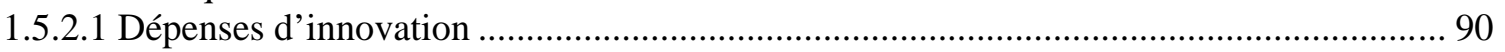

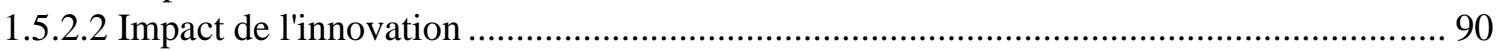

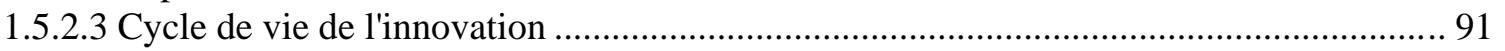

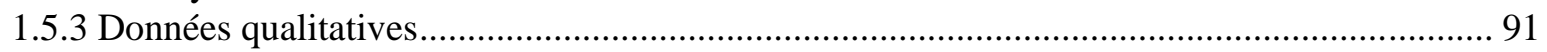

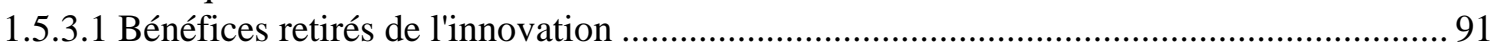

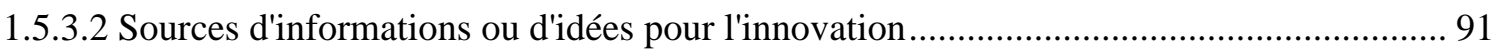

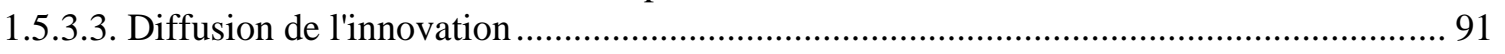

1.6 Période de référence à adopter dans l'approche objet .................................................................. 92

2. INDICATEURS D'OUTPUTS DE L'INNOVATION FONDÉS SUR LA PRESSE SPÉCIALISÉE 92

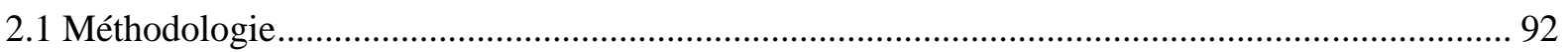

2.2. Avantages et inconvénients de cette méthode ............................................................................... 95

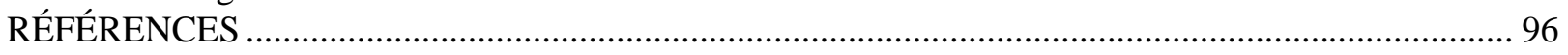

ANNEXE 2 LA COLLECTE DES DONNÉES SUR L'INNOVATION NON TECHNOLOGIQUE........ 97

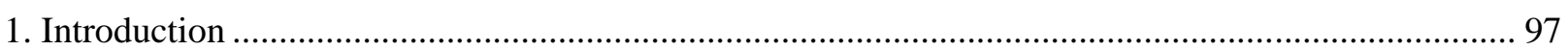

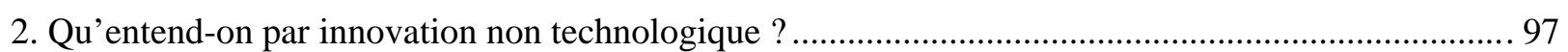

3. Expérience acquise dans la mesure de l'innovation non technologique ............................................. 98

4. Quelles sont les données qu'il convient de recueillir sur l'innovation non technologique ?.............. 98

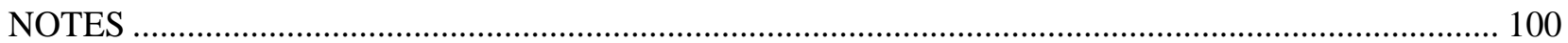




\section{Chapitre 1}

\section{OBJECTIFS ET PORTÉE DU MANUEL}

\section{INTRODUCTION}

1. Il est désormais couramment admis que la mise au point et la diffusion de nouvelles technologies jouent un rôle crucial dans la croissance de la production et de la productivité. Néanmoins, nos connaissances du processus d'innovation et de ses répercussions économiques sont encore insuffisantes à bien des égards. Ainsi, nous sommes manifestement au coeur d'une grande révolution technologique, l'économie mondiale se trouvant remodelée par les nouvelles technologies de l'information et par des transformations radicales dans des domaines comme la biotechnologie et la science des matériaux. Pourtant, ces mutations technologiques fondamentales ne se traduisent pas par des améliorations de la productivité globale des facteurs et des taux de croissance de la production.

2. En s'efforçant d'élucider ce mystère, on en est venu, ces dernières années, à mettre davantage l'accent sur l'importance critique de certains aspects du processus d'innovation autres que la R-D, dans la mesure, en particulier, où ils influent sur les taux de diffusion. Ce sont des domaines dans lesquels nous sommes aux prises avec de sérieux problèmes, notamment du fait de l'absence de données fiables et systématiques. Nous ne réussirons à affiner l'analyse de l'innovation et à résoudre les problèmes qu'elle pose au plan de l'action gouvernementale que si nous sommes, notamment, capables d'améliorer la disponibilité de l'information.

3. La première version de ce manuel, publiée en 1992, et les enquêtes qui ont été conduites sur ces bases, en particulier l'Enquête communautaire sur l'innovation (ECI) organisée par la Commission Européenne (CE), ont montré qu'il est possible de mettre au point et de rassembler des données sur l'innovation, processus complexe et diversifié.

4. Cette deuxième édition du manuel d'Oslo reprend le cadre initial de concepts, de définitions et de méthodologie qu'elle actualise en y intégrant l'expérience acquise à l'occasion des enquêtes, les progrès accomplis dans la compréhension du processus d'innovation ainsi qu'un éventail élargi de branches d'activité. Elle présente les principes directeurs à suivre pour mettre au point des indicateurs d'innovation comparables pour les pays de l'OCDE en même temps qu'elle examine les problèmes d'analyse et de fond liés à ces indicateurs. Le manuel poursuit un double objectif, à savoir tracer un cadre dans lequel il sera possible d'améliorer la comparabilité des enquêtes réalisées et aider les nouveaux venus à aborder cet important domaine.

5. Le présent chapitre a pour propos de donner un aperçu de la couverture et du contenu du manuel (voir encadré 1), pour aider ainsi les nouveaux venus et tous ceux qui ne sont pas des experts en la matière à utiliser le corps du texte, d'indiquer pourquoi certaines sortes de données sont ou ne sont pas rassemblées, ainsi que de signaler les principaux problèmes liés à l'établissement de normes à suivre pour arriver à des indicateurs comparables. 


\begin{tabular}{|l} 
Encadré 1. Plan du manuel \\
Le corps du manuel s'ouvre sur un examen général des aspects qui sont appelés à peser sur le \\
choix des indicateurs (chapitre 2) : \\
- $\quad$ une compréhension théorique adéquate de la structure et des caractéristiques du processus \\
d'innovation, ainsi que les conséquences pour l'élaboration des politiques ; \\
- $\quad$ les grands problèmes non résolus qu'un complément de données permettrait de clarifier ; \\
- les conséquences pour la portée du manuel. \\
Vient ensuite un exposé des définitions, critères et classifications qui sont pertinents pour des \\
- les définitions de base de l'innovation technologique de produits et de procédés - innovation \\
TPP - et des activités d'innovation TPP (chapitre 3); \\
- les classifications institutionnelles (chapitre 4). \\
Des suggestions et recommandations sont ensuite présentées au sujet de la conduite des enquêtes \\
nationales et internationales sur l'innovation TPP : \\
- la mesure de certains aspects du processus d'innovation TPP (chapitre 5); \\
- la mesure des dépenses consacrées à l'innovation TPP (chapitre 6); \\
- les procédures d'enquête sur l'innovation (chapitre 7). \\
Le manuel s'achève sur un ensemble d'annexes traitant d'aspects qui, soit offrent des options de \\
rechange pour les procédures généralement préconisées ou qui, tout en étant pertinents, ne sont pas \\
suffisamment élaborés pour figurer dans le corps du manuel : \\
- l'approche "objet" pour la compilation et la collecte de données (annexe 1); \\
- la collecte de données sur l'innovation non technologique (annexe 2).
\end{tabular}

2. FACTEURS INFLUENCANT LA PORTÉE DU MANUEL

6. Selon quels critères décidera-t-on de la portée, la structure, la terminologie, etc., à retenir pour une collecte de données comparables au plan international ? La diversité des sujets abordés dans les enquêtes lancées récemment à titre exploratoire démontre qu'il existe un large éventail de données potentiellement disponibles. Manifestement, une enquête couvrant la totalité du champ des investigations précédentes serait d'une lourdeur telle qu'elle serait impossible à réaliser dans la pratique. Il faut donc définir les priorités et choisir les sujets, les branches d'activité et les méthodes d'enquête à privilégier. Il convient aussi d'établir une distinction entre les données qu'il vaut mieux réunir sur une base régulière et les aspects qui peuvent être traités de façon plus efficace au moyen de projets ponctuels.

\subsection{Comprendre le processus d'innovation et ses conséquences pour la politique de l'innovation}

7. Les besoins en informations des décideurs politiques et des analystes sont un élément capital à prendre en compte pour l'élaboration des indicateurs d'innovation. Le chapitre 2 passe en revue ces besoins, lesquels relèvent du système général d'information qui concourt à atténuer les incertitudes pesant 
sur la prise des décisions et ont été influencés dès la première version de ce manuel par les évolutions de l'économie de l'innovation.

8. La politique de l'innovation, issue d'emprunts à la politique scientifique et technologique comme à la politique industrielle, est de création toute récente. Son apparition marque une prise de conscience grandissante du fait que le savoir, sous toutes ses formes, joue un rôle capital dans le progrès économique, que l'innovation est au coeur de cette "économie fondée sur le savoir" et aussi que l'innovation est un phénomène plus complexe et systémique qu'on ne l'avait d'abord imaginé. Les approches systémiques de l'innovation conduisent à déplacer l'accent pour le mettre sur les interactions entre les institutions, en examinant les processus interactifs au niveau tant de la création du savoir que de sa diffusion et de son application. L'expression "système national d'innovation" a été forgée pour cet ensemble d'institutions et de flux des connaissances.

9. Afin de parvenir à un cadre conceptuel pour ce manuel, au chapitre 2 on place au centre ce qui est décrit comme la "dynamo de l'innovation", les forces dynamiques façonnant l'innovation dans les firmes qui sont appuyées et influencées par les facteurs de transfert, par la base scientifique et technique ainsi que par les conditions-cadres.

10. Au chapitre 2 on approfondit la notion de dynamo de l'innovation et on examine la portée économique du progrès technologique et les théories associées. Comme dans la version précédente du manuel, ce chapitre porte essentiellement sur l'innovation au niveau de la firme, plus particulièrement sur l'approche néo-schumpétérienne et le modèle d'innovation se fondant sur les liaisons en chaîne, dans lequel l'innovation est vue sous l'angle des interactions entre, d'un côté les possibilités offertes par le marché et, de l'autre, la base de connaissances et les aptitudes de la firme. Cependant, la question n'est pas d'adhérer à tel ou tel modèle particulier d'innovation, mais plutôt de montrer que l'innovation est une activité complexe, diversifiée et aux multiples composantes agissant les unes sur les autres, et que les sources de données doivent refléter ces caractéristiques.

11. Pour les besoins des décideurs et des analystes évoqués ci-dessus, à la fin du chapitre 2 on recense six grands domaines d'étude : les stratégies de la firme, le rôle de la diffusion, les sources de l'information nécessaire pour l'innovation et les obstacles à l'innovation, les inputs de l'innovation, le rôle des pouvoirs publics dans l'innovation industrielle et les outputs de l'innovation.

\subsection{Expérience acquise au niveau de la fourniture de données}

12. La première édition du manuel a été testée dans des enquêtes réalisées dans un large éventail de pays de l'OCDE. La plupart d'entre elles ont été menées dans le cadre de l'Enquête communautaire sur l'innovation (ECI), lancée conjointement par Eurostat et la DGXIII [programme SPRINT, Observatoire de l'innovation en Europe (OIE)]. Cette enquête reposait sur un questionnaire établi en commun à partir de la première version du manuel. Treize pays, représentés par des entrepreneurs nationaux, ont pris part à l'exercice (Allemagne, Belgique, Danemark, Espagne, États-Unis, France, Grèce, Irlande, Italie, Luxembourg, Norvège, Pays-Bas et Portugal), qui couvrait l'innovation technologique dans les industries manufacturières. Une large expérience a pu ainsi être acquise du fait que les organisations ayant participé à l'ECI se caractérisaient par une grande variété d'expertises, ce qui a permis de couvrir toute une série de méthodes et d'approches (voir Evaluation de l'enquête ECI - Phase I, publication OIE N ${ }^{\circ} 11$ ). Au moment de la rédaction du présent manuel, des études pilotes étaient en cours sur l'innovation technologique dans le secteur des services. 
13. Dans leur majorité, les autres pays de l'OCDE ont également testé les concepts et classifications figurant dans la première édition du Manuel d'Oslo à l'occasion d'enquêtes complètes ou partielles, avec des résultats plus ou moins bons selon les types de question. Cette deuxième version du manuel repose ainsi sur une vaste expérience concrète de l'aptitude des firmes à comprendre et à appliquer les concepts en cause, de l'exécution pratique des enquêtes ainsi que des problèmes posés par la compilation et l'interprétation des données obtenues.

\section{PORTÉE DU MANUEL}

14. Pour les raisons présentées à la fin du chapitre 2 :

- le manuel porte uniquement sur l'innovation dans le secteur des entreprises ;

- il concerne l'innovation au niveau de la firme ;

- il est centré sur l'innovation technologique de produit et procédé (innovation TPP), en proposant également des lignes directrices facultatives pour d'autres formes d'innovation telles que le changement organisationnel ;

- il couvre la diffusion jusqu' au stade "nouveau pour la firme".

\subsection{Couverture sectorielle}

15. L'innovation peut, bien entendu, intervenir dans n'importe quel secteur de l'économie, y compris dans les services publics, par exemple dans le domaine de la santé ou de l'éducation. Les principes directeurs présentés dans le manuel concernent essentiellement les innovations du secteur des entreprises dans les domaines, plus particulièrement, de l'industrie manufacturière, du bâtiment, de la production et distribution d'électricité, de gaz et d'eau et dans le domaine des services commercialisés. Ils ont été abondamment testés pour les industries manufacturières dans l'enquête ECI et dans des enquêtes analogues réalisées dans d'autres pays Membres de l'OCDE.

16. L'innovation dans le secteur des services, thème qui n'avait pas été abordé dans la première version du manuel, est complexe et présente des caractéristiques particulières décrites à la fin du chapitre 2. Jusqu'ici, elle n'a fait l'objet que d'enquêtes individuelles, réalisées pour la plupart à titre pilote, et les recommandations formulées à cet égard dans cette version du manuel reposent sur des bases moins solides que dans le cas des industries manufacturières.

\subsection{L'innovation au niveau de la firme}

17. Le manuel est centré sur les changements qui se produisent au niveau de la firme considérée individuellement. Il laisse de côté quelques autres catégories d'innovation examinées, par exemple, par Schumpeter, telles que l'ouverture d'un nouveau marché, la conquête de nouvelles sources d'approvisionnement en matières premières ou en biens semi-ouvrés, ou la réorganisation d'une industrie.

18. Le terme générique "firme" est utilisé pour les besoins des trois premiers chapitres du manuel. Une définition statistique spécifique en est donnée au chapitre 4 qui est consacré aux classifications. La définition exacte utilisée dans telle ou telle enquête ou étude peut avoir des répercussions sur les résultats. 
Dans le cas des sociétés multinationales, l'organisation des filiales n'est pas toujours la même. Une innovation donnée peut être introduite pays par pays ou marché par marché, ou bien encore elle peut être introduite simultanément dans le groupe tout entier. Les choses se compliquent encore lorsque les filiales sont en fait des franchisées et sont donc considérées comme des entreprises distinctes aux fins de l'enquête (ce qui est fréquemment le cas dans le secteur des services).

\subsection{Innovation technologique de produit et de procédé - innovation TPP}

19. Une entreprise peut apporter bien des changements à ses méthodes de travail, à l'utilisation qu'elle fait des facteurs de production et aux types de produits, dans le but d'améliorer sa productivité et/ou ses résultats commerciaux. Il serait mal commode, sous l'angle à la fois de la collecte des données et de leur analyse ultérieure, de se lancer dans une enquête exhaustive de ce genre de changements.

20. Diverses méthodes analytiques s'offrent pour choisir un sous-ensemble de changements à soumettre à une étude approfondie, par exemple tous ceux qui se rapportent à la diffusion des technologies de l'information - TI, ou ceux qui font intervenir des investissements immatériels (R-D, logiciels, formation, commercialisation, etc.) (voir la section 6 ci-après). Le manuel ne traite que des changements qui impliquent un degré élevé de nouveauté pour la firme. Il laisse de côté les changements qui "reviennent plus ou moins au même", par exemple l'achat de nouvelles copies d'un équipement TI d'un modèle déjà installé ailleurs dans la firme.

21. Le corps du texte est centré sur les produits (biens et services) et procédés nouveaux ou considérablement améliorés. On s'accorde à reconnaître que l'innovation purement organisationnelle est un phénomène répandu et peut déboucher sur une nette amélioration des résultats de la firme ${ }^{1}$. Cependant, ce thème n'est abordé pour l'instant que dans une annexe (annexe 2) étant donné le peu d'expérience pratique existant dans ce domaine.

22. Le corps du manuel traite des produits et procédés "technologiquement" nouveaux ou améliorés. La signification du qualificatif "technologique", tel qu'il s'applique aux produits et procédés, ainsi que sa portée exacte dans les enquêtes et études, n'apparaît pas toujours clairement. Tel est notamment le cas dans un contexte international. Il n'est pas toujours facile de faire une distinction entre le sens auquel ce terme est entendu ici, les définitions données dans les dictionnaires (ou l'équivalent le plus proche dans certaines langues) et qui peuvent varier de manière subtile d'un pays à l'autre, et les nuances auxquelles les répondants peuvent réagir. Par exemple, on a le sentiment que, dans le secteur des services, le terme "technologique" pourrait être entendu au sens de "employant des installations et matériels de haute technologie".

23. Au chapitre 3, on propose des définitions appuyées par des exemples. Il suffit, pour les besoins de l'examen liminaire des chapitres 1 et 2 , de donner une description pratique de l'innovation technologique de produit ou de procédé - TPP.

24. On entend par innovation technologique de produit la mise au point/commercialisation d'un produit plus performant dans le but de fournir au consommateur des services objectivement nouveaux ou améliorés. Par innovation technologique de procédé, on entend la mise au point/adoption de méthodes de production ou de distribution nouvelles ou notablement améliorées. Elle peut faire intervenir des changements affectant - séparément ou simultanément - les matériels, les ressources humaines ou les méthodes de travail. 
25. La distinction entre la nouveauté "technologique" et d'autres améliorations repose en grande part sur les "caractéristiques de performance" des produits et procédés en cause, et la possibilité de faire cette distinction en pratique dépendra de la mesure dans laquelle ces caractéristiques et leur degré de nouveauté constituent un facteur important des ventes de la firme/branche d'activité concernée. Par exemple, elle est plus facile à saisir et à appliquer aux biens et services faisant l'objet d'échanges entre les firmes, notamment dans les industries de haute technologie, qu'aux services et aux biens de consommation. On peut aisément imaginer un ensemble de caractéristiques de performance lorsqu'il s'agit de puces d'ordinateur, d'ordinateurs, d'usines de papeterie, de granulés plastiques ou même de services informatiques ou d'assurance commerciale et il est possible de se référer à certaines conventions au sujet, à tout le moins, de ce qui constitue des caractéristiques "nouvelles ou améliorées" jugées compréhensibles à la fois pour le vendeur et pour l'acheteur. Mais quelles sont les "caractéristiques objectives de performance" d'un repas italien (ou chinois), d'une représentation (ou d'un disque compact) de Ä̈da, d'une cravate (dessinée par un créateur, fabriquée par un contrefacteur, vendue dans un magasin à succursales multiples, etc.), d'une paire de baskets, d'un pot de crème pour le visage ou d'un baril de lessive en poudre? Jusqu'à quel point les consommateurs prennent-ils leurs décisions d'achat en se fondant sur les "caractéristiques de performance" ? Jusqu'à quel point les producteurs identifient-ils les produits nouveaux sous cet angle "technologique" ?

26. Faute de critères permettant de répondre à ces questions, l'innovation technologique de produit, telle qu'elle est définie dans le manuel, exclut les changements apportés à des produits qui donnent à l'acheteur un sentiment subjectif de plus grande satisfaction en raison de ses propres goûts et jugements esthétiques, ou qui répondent au souci de suivre la mode, ou encore qui sont pour beaucoup le fruit de campagnes de marketing. Cependant, comme ces changements ont une importance extrême pour certaines branches et font intervenir des activités identiques ou similaires à l'innovation TPP (conception, marketing, etc.), ils sont identifiés séparément sous la rubrique "Autres améliorations créatives de produits".

\subsection{Diffusion de l'innovation}

27. Par "diffusion", on entend la manière dont, depuis leur première application mondiale, les innovations TPP se répandent - par l'intermédiaire des mécanismes du marché ou autrement - dans d'autres pays et régions, ainsi que dans différentes industries, marchés et firmes. Sans diffusion, une innovation TPP n'aura pas d'incidences économiques. Afin de prendre en compte ne serait-ce que partiellement cet aspect concernant la diffusion, comme cela est recommandé au chapitre 2, il a été décidé qu'une innovation doit être au minimum "nouvelle pour la firme" pour être prise en compte dans le système décrit dans le manuel. Cela revient à dire que la diffusion complète, à travers une firme, d'une technologie nouvelle après sa première adoption/commercialisation n'est pas prise en considération.

28. Toutefois, parce qu'il couvre tous les produits et procédés présentant des caractéristiques de performance nouvelles pour la firme, l'exercice va beaucoup plus loin que certaines études antérieures centrées sur les technologies clés et leur première introduction au niveau mondial et, parfois, national.

29. Il est également proposé (chapitre 5) de demander aux entreprises innovantes d'indiquer les branches d'activité qui utiliseront probablement leurs produits technologiquement nouveaux ou améliorés. Au chapitre 2 il est fait mention d'autres méthodes permettant d'obtenir des données sur la diffusion de la technologie. 


\section{DONNÉES A FOURNIR SUR LES PRINCIPALES QUESTIONS}

\subsection{Facteurs influant sur l'innovation TPP}

30. Les stratégies des entreprises, les sources d'informations nécessaires pour innover, ainsi que les obstacles à l'innovation, sont les principales questions qui sont proposées pour examen au chapitre 2. Des séries de questions conçues pour obtenir des informations sur chacun de ces thèmes sont présentées au chapitre 5. Chacune s'accompagne d'une liste éventuelle de facteurs. Il est recommandé que les points de vue des entreprises soient enregistrés sous forme binaire (important/pas important) ou selon un nombre réduit d'options (de très important à sans intérêt). Cette approche semi-quantitative a été choisie en raison de sa commodité pour les répondants bien que ses résultats soient difficiles à analyser. Les listes données sous chaque thème ont été révisées sur la base des résultats de l'enquête ECI.

\subsection{Activités et dépenses d'innovation TPP}

31. Par activités d'innovation TPP, on entend l'ensemble des démarches scientifiques, technologiques, organisationnelles, financières et commerciales qui aboutissent, ou sont censées aboutir, à la réalisation de produits ou procédés technologiquement nouveaux ou améliorés. Certaines peuvent être intrinsèquement innovantes, d'autres ne le sont pas véritablement, mais elles sont indispensables pour arriver à un résultat concret.

32. Diverses études politiques ou analytiques évoquées au chapitre 2, fournissent des ventilations de ces activités correspondant aux points de vue personnels de leurs auteurs. L'établissement d'une liste unique à des fins statistiques fait donc problème, d'autant plus qu'il faut aussi tenir compte de la capacité des entreprises concernées à réfléchir aux données qu'elles auront à fournir dans cette optique.

33. Qui plus est, l'innovation n'est pas un processus linéaire et il se pourrait que le système comporte d'importants allers et retours (voir le chapitre 2). Elle fait intervenir les principales activités suivantes: des travaux de R-D, d'autres moyens d'acquisition des connaissances (brevets, licences, services techniques, etc.), l'achat de machines et de matériels (qui incorporent une technologie nouvelle ou qui sont destinés à être utilisés de manière habituelle pour la fabrication d'un produit nouveau), diverses autres mesures tendant à préparer la production et la livraison, y compris l'outillage, la formation du personnel et des actions de marketing (voir le chapitre 3). De toutes ces activités, celles qui relèvent automatiquement de l'innovation TPP sont la R-D et l'achat de machines incorporant une technologie nouvelle. Les autres n'en font partie que si elles sont indispensables pour réaliser des innovations TPP, mais pas si elles sont effectuées en liaison avec une innovation purement organisationnelle, d'autres améliorations créatives ou une simple expansion des capacités ou de la production.

34. On pourrait dire que, durant une période donnée, les activités d'innovation d'une entreprise entrent dans l'une ou l'autre des trois catégories suivantes :

- Activité réussie débouchant sur la réalisation d'un produit ou procédé technologiquement nouveau ou amélioré.

- Activité avortée avant la réalisation d'un produit ou d'un procédé technologiquement nouveau ou amélioré, parce que le projet rencontre des difficultés, ou parce que l'idée et le savoir-faire sont vendus ou transmis d'une autre manière à une autre entreprise, ou encore parce que le marché a changé. 
- Activité en cours, c'est-à-dire qu'elle se poursuit sans avoir encore abouti à des résultats concrets.

35. Les dépenses sont chiffrées sur la base de la somme de ces trois catégories d'activité au cours d'une période de temps donnée (voir le chapitre 6). Ou bien on peut réunir des informations sur le total des dépenses consacrées à des activités conduisant à des innovations individuelles. Quelle que soit la formule adoptée, les entreprises semblent avoir beaucoup de mal à fournir un ensemble complet de données. Or, ces données sont d'une importance capitale pour procéder à une analyse économique, ne serait-ce que pour mesurer la part des dépenses de R-D dans le coût total des produits et procédés nouveaux auxquels la R-D est censée apporter une contribution. On espère que, au fur et à mesure qu'elles se livreront à ces exercices, les entreprises finiront par se convaincre qu'il est de leur propre intérêt de chiffrer le coût de leurs activités d'innovation.

\subsection{L'entreprise innovante TPP et l'impact de l'innovation TPP}

36. Lorsqu'on présente les résultats d'une enquête sur l'innovation, on commence notamment par la proportion d'entreprises "innovantes" par opposition à celles considérées comme "non innovantes". Cette proportion risque de devenir un "nombre magique" comparable au pourcentage du PIB consacré à la R-D. C'est un chiffre qui doit être présenté et interprété avec une certaine prudence. Tout d'abord, le meilleur moyen de le calculer est de croiser, dans la mesure du possible, les structures industrielles avec une ventilation des entreprises par taille; un chiffre global peut être très trompeur (les ventilations qu'il convient d'effectuer sont recommandées au chapitre 4). Qui plus est, il importe de veiller à ce que seules les innovations TPP soient considérées, à l'exclusion des innovations purement organisationnelles ou même d'autres améliorations créatives. La définition de la firme que l'on retient peut avoir elle aussi son importance. Le cas des entreprises franchisées, évoqué plus haut, en est un exemple. Les points de distribution d'une chaîne de développement express des photographies doivent-ils être considérés comme des entreprises innovantes s'ils sont tous équipés de matériels comportant les mêmes améliorations?

37. L'entreprise innovante TPP (au sens défini au chapitre 3) est celle qui a mis au point des produits, des procédés ou des associations de produits et procédés technologiquement nouveaux ou notablement améliorés au cours de la période considérée. C'est une entreprise dont les activités d'innovation ont réussi durant cette période. A l'intérieur de cette catégorie, il peut être intéressant de distinguer les entreprises dont les innovations TPP ont un caractère purement "passif", c'est-à-dire les entreprises dont l'innovation a consisté exclusivement à importer une technologie incorporée dans des machines et équipements nouveaux. De plus, dans le cas des sociétés créées durant la période considérée, il est recommandé d'inclure dans la catégorie des entreprises innovantes uniquement celles qui ont introduit une innovation de produit ou de procédé nouveau pour le marché sur lequel elles opèrent au moment de leur création, ainsi que celles qui ont introduit une innovation TPP après leur création (le chapitre 6 présente des principes directeurs plus détaillés à ce sujet).

38. L'impact d'une innovation TPP peut se mesurer au pourcentage de ventes générées par des produits nouveaux ou améliorés (comme on l'indique au chapitre 5). Là encore, l'interprétation de l'indicateur en résultant présente des difficultés. Pour les entreprises qui ont démarré au cours de la période (triennale) considérée, tous les produits sont en principe nouveaux et le pourcentage de ventes découlant des produits nouveaux ou améliorés est de 100 pour cent par définition. La section 6 du chapitre 3 propose une convention qui permet de parer à cette distorsion.

39. L'indicateur est également influencé par la longueur du cycle de vie du produit. Le besoin d'innovation se fait sentir plus fréquemment pour les groupes de produits à cycle de vie relativement court que pour les groupes de produits à cycle de vie plus long. 
40. Il est également suggéré dans le chapitre 5 de réunir des éléments d'information sur l'impact des innovations TPP sur les facteurs de production.

\section{QUELQUES QUESTIONS A PROPOS DES ENQUÊTES}

\subsection{Approches de la collecte des données}

41. Il existe deux grandes approches de la collecte de données relatives aux innovations TPP des firmes : "l'approche sujet" qui part du comportement et des activités d'innovation de l'ensemble de la firme et "l'approche objet" qui est centrée sur le nombre et les caractéristiques des innovations considérées individuellement.

42. Il avait été décidé, au moment de la préparation de la première version du manuel, que l'approche sujet se prêtait mieux à une normalisation internationale et on l'avait donc retenue comme fondement des principes directeurs initiaux. Cependant, devant les avantages que présente l'approche objet pour certains types de questions (ou enquêtes), les définitions de base du chapitre 3 et les classifications institutionnelles du chapitre 4 de la présente version ont été formulées de manière à convenir aux deux approches et une annexe spéciale a été consacrée à l'approche objet (annexe 1). Les précisions données dans les chapitres 5 à 7 sont essentiellement destinées aux enquêtes sur l'innovation fondées sur la firme (l'approche sujet), mais elles peuvent aussi présenter un intérêt pour d'autres types d'enquêtes.

\subsection{Méthodes d'enquête}

43. Pour que les enquêtes sur l'innovation fondées sur l'entreprise soient comparables à l'échelle internationale, il est important d'harmoniser leurs principales caractéristiques, autant que faire se peut. Des principes directeurs sont donnés à cet effet au chapitre 7.

44. La population cible des enquêtes sur l'innovation devrait être la plus homogène possible. Lorsqu'il s'agit d'enquêtes par sondage, les échantillons doivent être aussi représentatifs que possible de la population cible. Toutes les grandes entreprises appartenant à la population cible doivent être incluses dans l'enquête. Quant aux entreprises plus petites, il y a lieu d'établir un échantillon aléatoire stratifié selon la taille et la branche d'activité.

45. Afin d'obtenir un taux de réponses satisfaisant, le questionnaire doit être aussi bref que possible, avec des questions et instructions clairement libellées. Cet impératif peut conduire à exprimer les définitions formelles du chapitre 3 sous une forme plus intelligible adaptée aux répondants de la branche d'activité concernée, notamment lorsqu'il s'agit des industries de service.

46. Durant la phase de collecte des données, il convient de s'attacher tout particulièrement à vérifier la fiabilité et la cohérence des données ainsi qu'aux procédures de rappel. La comparabilité internationale des données obtenues sera d'autant plus grande que des méthodes uniformes auront été adoptées en ce qui concerne l'imputation des valeurs manquantes, les facteurs de pondération, les principes à suivre pour la présentation des résultats, etc. 


\section{LES RELATIONS ENTRE LE MANUEL D'OSLO, D'AUTRES NORMES INTERNATIONALES ET CONCEPTS CONNEXES}

47. L'innovation se produit à travers toute l'économie mais elle présente certaines caractéristiques particulières qui la distinguent des activités plus typiquement scientifiques et technologiques qu'elle fait habituellement intervenir comme des activités économiques dont elle fait partie intégrante.

\subsection{Activités de S-T : La famille des manuels de Frascati}

48. Il existe deux grandes familles d'indicateurs de S-T qui intéressent directement la mesure de l'innovation TPP : les ressources consacrées à la R-D et les statistiques sur les brevets.

49. Les données concernant la R-D sont collectées au moyen d'enquêtes nationales réalisées selon les lignes directrices énoncées dans le Manuel de Frascati (OCDE, 1993). Elles se sont révélées précieuses dans bon nombre d'études : par exemple, les effets de la R-D sur la productivité ont pu être mesurés par des techniques économétriques au niveau du pays, du secteur et de l'entreprise. Ces données connaissent deux grandes limitations. Tout d'abord, la R-D est un input. Elle est manifestement liée au progrès technique mais elle ne le mesure pas. En second lieu, la R-D n'englobe pas l'ensemble des efforts faits par les firmes et les pouvoirs publics dans ce domaine car il existe d'autres sources de progrès techniques, par exemple l'apprentissage par la pratique, qui échappent à cette définition étroite.

50. Un brevet est un droit de propriété juridique sur une invention, qui est délivré par les bureaux de brevets nationaux. Il confère à son titulaire un monopole (d'une durée limitée) d'exploitation de l'invention brevetée, en contrepartie de la divulgation de la découverte (en vue d'en permettre une utilisation collective plus large). Ceux qui étudient la technologie utilisent de plus en plus et de bien des manières les statistiques de brevets que l'on peut considérer comme des indicateurs de l'output des activités d'invention. Le nombre de brevets délivrés à une entreprise ou à un pays donné peut être le reflet de son dynamisme technologique ; l'examen des technologies brevetées peut donner certaines indications quant aux orientations du progrès technologique. Considérer les brevets comme des indicateurs comporte des inconvénients bien connus. Nombre d'innovations ne correspondent pas à une invention brevetée. Les brevets attachés à une invention dont la valeur technologique et économique est quasiment nulle sont nombreux, alors que très peu ont une très grande valeur ; beaucoup de brevets n'ont jamais débouché sur une innovation (voir Les données sur les brevets d'invention et leur utilisation comme indicateurs de la science et de la technologie, OCDE, 1994).

51. Ces deux grandes familles de statistiques sont complétées par diverses autres, parmi lesquelles on peut citer: les statistiques sur les publications scientifiques (bibliométrie), les articles parus dans des revues professionnelles et techniques (que l'on appelle "LBIO" - indicateurs d'output de l'innovation tirés de la presse spécialisée), la balance des paiements technologiques et l'activité dans les secteurs de haute technologie (investissements, emploi, commerce extérieur). De plus, des informations sur l'innovation et les activités d'innovation peuvent provenir indirectement de diverses autres sources, par exemple les enquêtes de conjoncture auprès des entrepreneurs ou les statistiques de l'enseignement.

52. Chaque fois que cela est possible, le manuel se fonde sur les concepts et classifications figurant dans d'autres volumes de la série de manuels de l'OCDE pour la mesure des activités scientifiques et technologiques (voir l'encadré 2) et tout particulièrement sur le Manuel de Frascati sur les ressources consacrées à la R-D (OCDE, 1993). Cette remarque s'applique tout spécialement à un certain nombre de questions supplémentaires sur la R-D et d'autres activités de S-T recommandées d'inclure dans les enquêtes sur l'innovation TPP aux chapitres 5 et 6 . 


\section{Encadré 2 - "Famille de Frascati" des principes directeurs pour la mesure des activités scientifiques et technologiques}

Méthode type proposée pour les enquêtes sur la recherche et le développement expérimental - Manuel de Frascati - Cinquième édition (OCDE : 1993)

Définitions et conventions de base pour la mesure de la recherche et du développement expérimental (R-D) Résumé du Manuel de Frascati (OCDE : GD1993)

Méthode type proposée pour le recueil et l'interprétation des données sur la balance des paiements technologiques - Manuel BPT (OCDE : GD1992)

Principes directeurs proposés par l'OCDE pour le recueil et l'interprétation des données sur l'innovation technologique - Manuel d'Oslo - deuxième édition (OCDE/CE/Eurostat : 1996)

Les données sur les brevets d'invention et leur utilisation comme indicateurs de la science et de la technologie - Manuel Brevet (OCDE/GD(1994)

La mesure des ressources humaines consacrées à la S-T - Manuel de Canberra

(OCDE/CE/Eurostat : GD1995)

\subsection{Autres normes et classifications économiques}

53. Etant donné qu'il faut situer l'innovation dans un contexte plus large, tant du point de vue théorique que sous l'angle des bases de données, on a utilisé dans toute la mesure du possible les lignes directrices et classifications de l'Organisation des Nations Unies, notamment le Système révisé de comptabilité nationale - SCN (CCE et al., 1994) et la Classification internationale type, par industrie, de toutes les branches d'activité économique - CITI Rév. 3 (ONU, 1990) plus, pour les besoins de ce manuel commun OCDE/CE, les normes européennes correspondantes, notamment la Nomenclature générale des activités économiques dans les Communautés européennes - NACE Rév. 1 - série 2E.

\subsection{Autres concepts et enquêtes connexes}

54. Comme on l'a remarqué ci-dessus, il existe bien d'autres manières d'examiner les changements intervenant dans les firmes qui améliorent leur productivité et leurs résultats. Nous n'en retiendrons ici que deux : l'investissement immatériel ; la création et l'adoption des technologies de l'information - TI.

55. L'investissement immatériel recouvre toutes les dépenses courantes qu'une firme engage pour son développement et dont les résultats escomptés se feront sentir au-delà de l'année où elles sont encourues. Il n'existe pas de définition type, mais on admet généralement que ce concept recouvre, en plus des dépenses courantes de R-D, les dépenses engagées pour des opérations ponctuelles de marketing, de formation, de mise au point de logiciels et pour d'autres aspects du même ordre. Ce concept couvre non 
seulement les dépenses courantes affectées à l'innovation TPP, mais aussi des postes qui n'entrent pas dans les dépenses courantes pour l'innovation TPP (en font partie, par exemple, toutes les dépenses consacrées par la firme à la formation et au marketing d'une manière générale et pas simplement les activités de formation ou de marketing réalisées en liaison avec le lancement de produits et procédés technologiquement nouveaux). Il exclut les investissements physiques tels que les dépenses en capital pour l'innovation TPP, dans lesquelles entrent les dépenses en capital de R-D, l'acquisition de machines et matériels nouveaux liés à des innovations TPP.

56. Les technologies de l'information comprennent à la fois des matériels et des logiciels. On pense que leur développement et leur diffusion ont eu un impact déterminant sur les profils de production et d'emploi dans un large éventail d'activités économiques. Pour ce qui est des matériels, il peut être intéressant de connaître non seulement la date à laquelle une société innove en lançant un matériel de TI technologiquement nouveau ou amélioré mais également la proportion de TI entrant dans son stock total d'équipement, y compris les achats ultérieurs de nouvelles machines du même modèle. On peut obtenir ce genre de renseignements grâce à des enquêtes spéciales sur les TI, enquêtes qui s'inscrivent dans le prolongement de celles qui ont été menées précédemment sur la "technologie de fabrication" (US Bureau of the Census 1988 \& 1992 ; Statistiques Canada, 1989 \& 1992/93 ; Australian Bureau of Statistics, 1993).

57. Dresser la carte du développement, de la production, de l'adaptation et de l'utilisation des logiciels est une affaire plus compliquée car il s'agit là d'activités qui sont menées à travers toute l'économie. Une catégorie spéciale a été définie à cette fin dans le Système de comptabilité nationale (CE et al., 1993) et des lignes directrices ont été établies pour les enquêtes relatives à l'industrie des services informatiques (ONU, 1992) mais la collecte des données correspondantes n'est pas encore entièrement au point.

\section{REMARQUE FINALE}

58. Ce manuel repose sur des points de vue consensuels sur la demande d'indicateurs d'innovation, ainsi que les besoins des décideurs politiques et la théorie économique qui la sous-tendent, les définitions et la couverture de l'innovation, ainsi que les enseignements à tirer des enquêtes précédentes. Géré conjointement par l'OCDE et la Commission européenne, il a été écrit pour et par des experts d'une trentaine de pays chargés de recueillir et d'analyser les données concernant l'innovation. Pour parvenir à ce consensus, il a parfois fallu accepter des compromis et se mettre d'accord sur des conventions. De plus, il est difficile d'énoncer des lignes directrices tout à fait rigoureuses en raison de la complexité même du processus d'innovation. Néanmoins, le manuel présente un ensemble solide de principes directeurs qui peuvent être appliqués pour établir des indicateurs significatifs de l'innovation technologique de produits et procédés. 


\section{Chapitre 2}

\section{LA NÉCESSITÉ DE MESURER L'INNOVATION}

\section{INTRODUCTION}

59. L'expression "l'économie fondée sur le savoir" a été forgée pour décrire les tendances de la plupart des économies avancées à devenir de plus en plus tributaire du savoir, de l'information et des compétences de haut niveau, ainsi que le besoin de plus en plus impérieux de disposer aisément de tous ces éléments. L'importance de ces tendances pour l'action des pouvoirs publics est mise en relief dans une étude majeure de l'OCDE ${ }^{2}$ :

"De nos jours, le savoir sous toutes ses formes joue un rôle capital dans le fonctionnement de l'économie. Les nations qui exploitent et gèrent efficacement leur capital de connaissances sont celles qui affichent les meilleures performances. Les entreprises qui possèdent plus de connaissances obtiennent systématiquement de meilleurs résultats. Les personnes les plus instruites s'adjugent les emplois les mieux rémunérés. Ce rôle stratégique du savoir explique l'accroissement de l'investissement dans la recherche et le développement, dans la formation et l'enseignement, ainsi que des autres investissements immatériels qui, dans la plupart des pays, se sont développés plus rapidement que les investissements dans le capital physique au cours des dernières décennies. Dans leur action, les pouvoirs publics doivent donc mettre l'accent sur le renforcement de la capacité d'innovation, de création et d'utilisation des connaissances de nos économies. Le changement technique résulte des efforts d'innovation, notamment des investissements immatériels, tels que la R-D, et ouvre des opportunités d'investissement ultérieur dans les capacités de production. C'est pourquoi, à terme, il est générateur d'emplois et d'accroissement des revenus. L'une des tâches essentielles des pouvoirs publics est de mettre en place des conditions qui incitent les entreprises à engager les investissements et les efforts d'innovation indispensables à la poursuite du progrès technique."

60. On considère que l'innovation joue un rôle central dans l'économie fondée sur le savoir, mais les processus complexes d'innovation n'étaient pas suffisamment bien cernés jusqu'à une date récente. Cependant, ils sont maintenant mieux compris grâce aux nombreuses études réalisées ces dernières années $^{3}$. Au niveau macroéconomique, il existe un solide réseau de faits montrant que l'innovation est le facteur dominant de la croissance économique nationale et de la spécialisation commerciale des pays. Au niveau microéconomique - c'est-à-dire au sein des entreprises - la R-D apparait comme un élément qui renforce l'aptitude d'une entreprise à assimiler et utiliser toutes sortes de connaissances nouvelles et pas seulement des connaissances technologiques.

61. D'autres facteurs qui influent sur l'aptitude des firmes à apprendre sont jugés essentiels. Une grande importance est attachée à la commodité des moyens de communication, à l'efficacité des canaux d'information, à la transmission des qualifications et à l'accumulation du savoir, tant au sein des organisations qu'entre elles. En particulier, la gestion et une stratégie prospective appropriée sont des facteurs cruciaux. Ils déterminent en grande part le champ des liaisons externes et les attitudes positives au sein de la firme qui favorisent un état d'esprit ouvert à l'adoption de meilleures pratiques et d'une meilleure technologie. Selon un récent Livre vert sur l'innovation de la Commission européenne ${ }^{4}$ : 
"L'entreprise innovante présente donc un certain nombre de caractéristiques qui peuvent être regroupées en deux grandes catégories de compétences :

- les compétences stratégiques : vision à long terme ; aptitude à identifier, voire anticiper les tendances du marché ; volonté et capacité de collecter, de traiter et d'intégrer l'information technologique et économique ;

- les compétences organisationnelles : goût et maîtrise du risque ; coopération interne, entre les différents départements fonctionnels, et externe, avec la recherche publique, les cabinets conseils, les clients et les fournisseurs; implication de l'ensemble de l'entreprise dans le processus de changement et investissements en ressources humaines."

62. L'un des thèmes majeurs de l'action des pouvoirs publics dans la plupart des pays développés est d'oeuvrer à une prise de conscience plus aiguë de l'importance de l'innovation. Si elle est au premier chef une émanation de la politique scientifique et technologique, la politique de l'innovation emprunte aussi beaucoup de ses traits à la politique industrielle. Au fil des progrès marqués dans la compréhension de l'innovation, l'élaboration des politiques dans ce domaine a connu de profondes modifications. Initialement, on pensait que le progrès technologique était le fruit d'un processus purement linéaire, dont le point de départ était la recherche scientifique fondamentale et qui se déroulait en allant, d'étape en étape, vers la recherche plus nettement appliquée, incorporant la science dans des applications technologiques et intégrant la commercialisation. La science était considérée comme le moteur et il suffisait que les gouvernements aient une politique scientifique. De nouvelles réflexions sur l'innovation ont fait ressortir l'importance des systèmes et ont débouché sur une approche plus intégrée de l'énoncé des politiques touchant l'innovation.

\section{ASPECTS ÉCONOMIQUES DE L'INNOVATION}

63. L'innovation est au coeur du changement économique. Pour reprendre les termes de Schumpeter, les innovations "radicales" façonnent les grandes mutations du monde alors que les innovations "progressives" alimentent de manière continue le processus de changement. Schumpeter a proposé une liste de divers types d'innovations ${ }^{5}$ :

i) l'introduction d'un nouveau produit ou une modification qualitative d'un produit existant ;

ii) l'introduction d'un procédé constituant une innovation pour une industrie ;

iii) l'ouverture d'un nouveau marché ;

iv) le développement de sources nouvelles d'approvisionnement en matières premières ou en d'autres inputs ;

v) les évolutions de l'organisation industrielle.

64. Il est capital de comprendre pourquoi le changement technologique se produit, pourquoi les entreprises innovent. La raison, selon les travaux de Schumpeter, est que les entreprises cherchent à s'assurer des rentes. Un nouveau dispositif technologique procure certains avantages à l'innovateur. Lorsqu'il s'agit d'une innovation de procédé aboutissant à des gains de productivité, la firme bénéficie d'un avantage de coût sur ses concurrentes et elle est alors en mesure d'obtenir une meilleure marge bénéficiaire sur le prix du marché en vigueur ou, selon l'élasticité de la demande, elle peut tirer parti tout 
à la fois de ses prix plus bas et de sa marge bénéficiaire plus élevée pour gagner des parts de marché sur ses concurrents et chercher à se procurer de nouvelles rentes. Lorsqu'il s'agit d'une innovation de produits, la firme jouit d'une position de monopole grâce soit à un brevet (monopole légal) soit au temps que mettent ses concurrents à l'imiter. Forte de ce monopole, la firme est à même de fixer des prix supérieurs à ceux qui seraient possibles sur un marché concurrentiel et elle bénéficie donc d'une rente.

65. D'autres travaux ont mis l'accent sur l'intérêt qu'a une firme à prendre position par rapport à ses concurrentes. Les entreprises innovent pour préserver leur position concurrentielle et aussi pour se procurer un avantage compétitif. Une firme peut réagir à une innovation d'une concurrente en innovant elle-même pour ne pas perdre des parts de marché. Ou encore, elle peut prendre les devants et innover pour s'assurer sur le marché une position stratégique vis-à-vis de ses concurrentes, par exemple en élaborant et en s'efforçant d'imposer des normes techniques plus élevées pour les produits qu'elle fabrique.

66. Le changement technologique ne va pas sans heurts. Les technologies nouvelles sont en concurrence avec celles qui sont bien établies et, dans bien des cas, elles s'y substituent. Les processus de diffusion technologique sont souvent très lents et impliquent habituellement des améliorations progressives aux technologies nouvelles et déjà rodées. Au milieu de ces turbulences, des firmes nouvelles prennent la place de celles moins aptes à s'adapter. Le changement technologique entraîne une réallocation des ressources, y compris des ressources humaines, entre les secteurs et entre les entreprises. Comme Schumpeter l'a souligné, le changement technologique peut être synonyme de destruction créatrice. Il peut être aussi une source de soutien et d'avantages réciproques entre concurrents, producteurs et clients.

67. Une grande partie du savoir technologique présente les caractéristiques d'un bien public, étant donné que le mettre à la disposition de multiples utilisateurs revient moins cher que le créer et que, une fois diffusé, on ne peut plus en refuser l'accès à ces utilisateurs. Cette caractéristique pose deux grands problèmes aux innovateurs privés. Le premier a trait au retombées positives de l'innovation (externalités positives), c'est-à-dire au fait que la rentabilité sociale de l'innovation est généralement plus élevée que la rentabilité privée (les clients et les concurrents bénéficient des innovations d'une entreprise). Le second problème est une autre facette du premier - le savoir ne peut faire l'objet d'une appropriation. Dans ce cas, la firme ne peut retirer tous les bénéfices générés par son innovation, ce qui peut amoindrir l'incitation à investir dans des activités d'innovation. Ainsi, lorsque le savoir technologique a les caractéristiques d'un bien public, les forces du marché qui, autrement, inciteraient les entreprises à innover, ne jouent pas (défaillance du marché).

68. De nombreuses études ont tiré, à partir de cette base théorique, des données statistiques et des indicateurs qui se rapportent principalement au coût de l'innovation ainsi qu'aux taux de rentabilité privée et sociale des activités d'innovation. Dans ces travaux, la rentabilité privée des activités technologiques est calculée au moyen de méthodes économétriques faisant intervenir une estimation des fonctions de production qui rattachent les inputs et les outputs des activités d'innovation au niveau de la firme ou au niveau global. Dans la mesure où le savoir technologique présente les caractéristiques des biens collectifs, les politiques scientifiques et technologiques ont été conçues en vue de porter remède à cette diminution de l'incitation à innover, ainsi qu'à d'autres défaillances du marché tels que le risque et les coûts de transaction. Les principaux instruments utilisés à cette fin ont été le financement public direct de la recherche et, en particulier, de la recherche fondamentale (le gouvernement agissant en tant que fournisseur d'un bien public) et les brevets (droits de propriété).

69. On estime aussi de plus en plus que les connaissances technologiques présentent d'autres caractéristiques telles que le phénomène d'accumulation (d'où résulte une rentabilité accrue) et qu'elles 
influencent les forces dynamiques des marchés, de sorte que l'état d'équilibre est loin d'être atteint (la tendance allant à un éloignement et non à un rapprochement du point d'équilibre). De là sont nés les développements récents de "l'économie évolutionniste" et de "la nouvelle théorie de la croissance"

70. L'approche évolutionniste souligne l'importance de la variété et de la diversité de la technologie, ainsi que de la manière dont cette variété est transposée en possibilités et résultats technologiques. Ces éléments influent sur l'aptitude des entreprises à innover ainsi que sur les "trajectoires" ou orientations de l'innovation des entreprises. Une remarque corollaire est que les données statistiques doivent être présentées à un degré élevé de désagrégation, en étant fondées sur les compétences et aptitudes au niveau de la firme, la constitution de réseaux et "l'exploration" de la technologie. Il faut aussi que les données permettent de repérer les spécificités du système à divers niveaux, et qu'elles indiquent les types, niveaux et degrés d'efficacité des interactions entre les entreprises, notamment à travers l'adoption d'innovations, et les interactions avec d'autres institutions, à l'échelle tant nationale qu'internationale.

71. Le fait de voir l'innovation à un plus haut niveau, dans une approche systémique, met en relief l'importance du transfert et de la diffusion des idées, des compétences, du savoir, de l'information et de toutes sortes de signaux. Les voies et réseaux à travers lesquels circule l'information sont enracinés dans le contexte social, politique et culturel, ils sont puissamment orientés par le dispositif institutionnel qui leur impose ses contraintes. L'approche des "systèmes nationaux d'innovation" (SNI) étudie les entreprises innovantes dans la perspective des institutions extérieures, des politiques gouvernementales, des concurrents, des fournisseurs, des clients, des systèmes de valeurs ainsi que des pratiques sociales et culturelles qui en conditionnent le fonctionnement ${ }^{8}$.

72. Les approches systémiques de l'innovation conduisent les pouvoirs publics à mettre l'accent sur les interactions entre les institutions, en examinant les processus interactifs qui interviennent dans la création du savoir ainsi que dans sa diffusion et son application. Elles ont permis de mieux cerner l'importance des conditions générales, des réglementations et des grandes lignes de l'action gouvernementale dans lesquelles s'inscrit le fonctionnement des marchés - et, partant, le rôle incontournable que jouent les gouvernements en suivant l'évolution de ce cadre général et en cherchant à l'adapter étroitement aux circonstances. Par exemple, on s'accorde à penser qu'il convient de traiter les questions touchant la défaillance du système en même temps que les questions liées à la défaillance du marché. Une importante étude de l'OCDE conclut à cet égard' :

"Parmi les nombreux facteurs qui influent sur le comportement des entreprises, il convient de se pencher sur ceux qui sont modelés par les politiques gouvernementales. Une approche systémique s'impose car :

- il n'existe pas de réponse simple à des problèmes aussi complexes que ceux que pose la relation entre technologie et emploi dans une économie fondée sur le savoir ;

- pour être efficace, une stratégie gouvernementale doit englober un certain nombre d'actions macroéconomiques et structurelles ;

- la cohérence du programme d'action est une condition de succès et dépend de la validité du cadre conceptuel de référence, ainsi que de la qualité du processus d'élaboration des politiques." 


\section{VERS L'ÉLABORATION D'UN CADRE CONCEPTUEL}

73. La collecte de données quantitatives demande un cadre, formel ou non, permettant d'organiser et de comprendre ces données. Elle présuppose un certain nombre d'idées sur la nature du sujet, ses caractéristiques essentielles et ce qui est important ou non.

74. Les facteurs touchant directement l'innovation relèvent de trois grandes catégories. Il s'agit du monde des affaires ("les entreprises"), des institutions scientifiques et technologiques, ainsi que des questions relatives au transfert et à l'assimilation de la technologie, du savoir et des compétences. Par ailleurs, la gamme des possibilités d'innovation est influencée par un quatrième ensemble de facteurs l'environnement constitué par les institutions, les dispositions juridiques, le cadre macroéconomique et diverses autres conditions qui existent indépendamment de toute considération touchant l'innovation.

75. On peut représenter ces quatre grands groupes ou ordres de facteurs se rapportant à l'innovation sur une carte qui fait ressortir les domaines dans lesquels les pouvoirs publics peuvent exercer un effet de levier sur l'innovation des entreprises, ou les domaines qu'il y a lieu de prendre en compte au moment de la conception des initiatives à prendre. C'est là une manière de présenter le terrain politique sur lequel sera édifié un système national généralisé d'innovation. Si la documentation spécialisée met l'accent sur les systèmes nationaux, il est néanmoins clair que, dans bien des cas, ce même genre de considérations s'appliquent au niveau local comme au niveau transnational.

Figure 1. Une carte du terrain politique de l'innovation

\section{"CONDITIONS-CADRES”}

Conditions et institution générales déterminant

l'éventail des possibilités d'innovation

\section{FACTEURS DE TRANSFERT}

Facteurs humains, sociaux et culturels

influençant la transmission de l'information aux entreprises et leur apprentissage

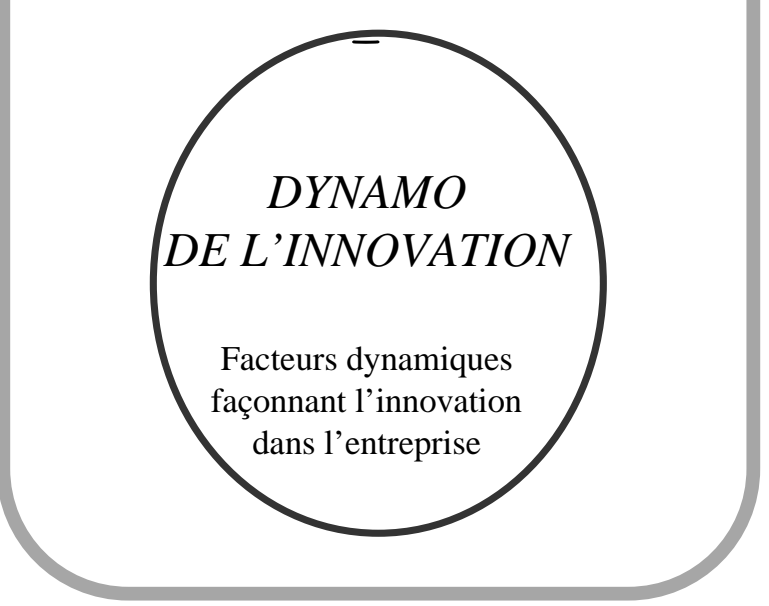




\section{BASE SCIENTIFIQUE ET TECHNIQUE}

Institutions scientifiques et technologiques constituant

le socle de la dynamo de l'innovation

76. Dans la carte $^{10}$ de la figure 1, les quatre grandes configurations du terrain politique de

l'innovation sont désignées comme suit:

- les conditions-cadres tracées par les facteurs institutionnels et structurels nationaux (juridiques, économiques, financiers et éducatifs) qui déterminent les règles du jeu et l'éventail des possibilités d'innovation ;

- la base scientifique et technologique - la somme accumulée de connaissances ainsi que les institutions scientifiques et technologiques qui sous-tendent l'innovation dans le secteur des entreprises en fournissant la formation technologique et les connaissances scientifiques, par exemple ;

- les facteurs de transfert sont ceux qui exercent une forte influence sur l'efficacité des liaisons, les courants d'informations et de compétences, ainsi que les mécanismes d'assimilation et d'apprentissage, sans lesquels les entreprises ne peuvent innover - il s'agit de facteurs ou d'agents humains dont la nature est déterminée de manière significative par les caractéristiques socioculturelles de la population; et

- la dynamo de l'innovation, foyer central de l'innovation dans les entreprises, est constituée par les facteurs dynamiques internes ou périphériques de la firme qui influent de manière très directe sur ses facultés d'innovation.

\subsection{Les conditions-cadres}

77. Le champ extérieur sur lequel les entreprises peuvent manoeuvrer et se transformer, qui constitue donc ainsi l'entourage immédiat des activités d'innovation au niveau de l'entreprise (la dynamo de l'innovation), comprend les institutions et conditions qui se sont créées (ou développées) pour l'essentiel pour des raisons sans rapport avec l'innovation. Ces facteurs déterminent les grands paramètres dans lesquels s'inscrivent l'existence et les activités des entreprises. Ils influent donc de manière sensible sur l'innovation dans le secteur des entreprises. Cet environnement institutionnel général trace les conditions-cadres à l'intérieur desquelles l'innovation peut se produire.

78. Les éléments constitutifs de ce cadre sont les suivants :

- le système éducatif de base de l'ensemble de la population, qui détermine les normes d'instruction minimales prévalant parmi la population active et sur le marché intérieur de la consommation ;

- l'infrastructure des communications, y compris les routes, les liaisons téléphoniques et les communications électroniques ;

- les institutions financières dont dépend, par exemple, la facilité d'accès au capital-risque ; 
- le contexte législatif et macroéconomique constitué notamment par la législation des brevets, la fiscalité, les règles de gestion des sociétés - ainsi que les politiques concernant les taux d'intérêts et de change, les droits de douane et la concurrence ;

- l'accessibilité du marché, y compris les possibilités de nouer des relations étroites avec les acheteurs, ainsi que les aspects tels que la taille et la commodité d'accès ;

- les structures industrielles et les conditions dans lesquelles s'exerce la concurrence, y compris l'existence de fournisseurs dans des branches d'activité complémentaires.

\subsection{La base scientifique et technique}

79. Les connaissances scientifiques et les compétences techniques constituent un point d'appui essentiel de l'innovation dans l'entreprise. Dans la plupart des pays, elles résident dans les institutions scientifiques et technologiques du secteur public qui s'emploient à les enrichir. La production mondiale de savoir scientifique émanant de ces institutions constitue un fondement conceptuel et théorique essentiel de l'innovation dans les firmes.

80. Il importe de cerner les différences fondamentales qui séparent les activités menées dans les institutions scientifiques et technologiques d'un côté et, de l'autre, dans les entreprises innovantes. Les motivations qui inspirent les membres de ces deux communautés sont très différentes. Il existe des différences dans la manière dont la réussite est reconnue, comme dans la manière dont elle est récompensée. Dans le domaine de la science, les individus tendent à exercer un rôle plus important que les institutions qui les emploient. D'un autre côté, "l'entreprise" (donc les aspects organisationnels tels que le travail en équipe et la stratégie) a habituellement plus d'importance que l'individu dans l'innovation et la technologie. Cependant, les réseaux d'individus - et, partant, de nombreux aspects du comportement social - sont d'une importance capitale dans le transfert d'informations à la fois entre scientifiques et entre ceux qui participent à l'innovation dans l'entreprise. Les institutions scientifiques et technologiques nationales peuvent constituer des filières locales efficaces d'accès à cette base et fournir le personnel qualifié nécessaire pour occuper les postes clés de l'innovation. Dans le cas de nombreuses innovations au niveau de la firme, elles sont aussi une mine d'avis spécialisés, elles offrent un cadre de collaboration et d'interactions fécondes, et elles sont une source d'importants progrès technologiques - qui ont souvent pour origine leurs propres besoins scientifiques en instruments perfectionnés.

81. Les composantes de la base scientifique et technique nationale sont notamment les suivantes :

- le système de formation technique spécialisée ;

- le système universitaire ;

- le dispositif d'appui de la recherche fondamentale (abstraction faite des percées radicales et des avantages à long terme, la recherche scientifique fondamentale apparaît parfois comme n'ayant que peu d'avantages directs pour l'innovation dans l'entreprise. Cependant, ses avantages indirects peuvent être très importants. L'investigation scientifique exige souvent la mise au point de matériels extrêmement complexes et ultrasensibles. Ainsi, de multiples domaines de la recherche fondamentale offrent un terrain fertile pour la formation de scientifiques qualifiés intéressés par les questions technologiques - dont l'expérience peut souvent être efficacement canalisée vers les problèmes industriels) ; 
- les activités de R-D intéressant les biens collectifs - les institutions et programmes de financement habituellement axés sur des domaines tels que la santé, l'environnement et la défense ;

- les activités de R-D stratégique - les institutions et programmes de financement axés directement sur la "R-D préconcurrentielle" ou les technologies génériques ; et

- le soutien de l'innovation ne pouvant faire l'objet d'une appropriation - institutions et programmes de financement directement axés sur la recherche menée dans des domaines où il est difficile pour les entreprises individuelles de s'approprier suffisamment les avantages issus de leurs propres travaux de recherche internes.

\subsection{Les facteurs de transfert}

82. La recherche sur l'innovation a permis de recenser un certain nombre de facteurs humains, sociaux et culturels qui sont d'une importance capitale pour le bon fonctionnement de l'innovation au niveau de l'entreprise. La plupart de ces facteurs tournent autour de la notion d'apprentissage. Ils concernent la facilité des communications à l'intérieur des organisations, les interactions informelles, la coopération et les voies de transmission de l'information et des compétences au sein des organisations et entre elles, ainsi que les facteurs sociaux et culturels qui exercent une influence omniprésente sur le degré d'efficience du fonctionnement de ces activités et voies de transmission. La recherche sur l'innovation a mis en relief un point capital, à savoir que la plus grande partie des connaissances essentielles, en particulier des connaissances technologiques, n'existe pas sous forme écrite. Ainsi, le transfert de certains types d'information ne peut se faire efficacement qu'entre deux personnes expérimentées - par la transmission à une personne réceptive suffisamment experte pour comprendre pleinement l'information, ou par le transfert physique des personnes qui sont porteuses du savoir. C'est l'apprentissage par toute la firme (c'est-à-dire la diffusion du savoir à un large éventail du personnel clé de la firme) qui constitue l'élément critique de l'aptitude de l'entreprise à innover. ${ }^{11}$

83. Globalement, on peut dresser la liste suivante des facteurs de transfert :

- les liaisons interentreprises formelles et informelles, y compris les réseaux de petites entreprises, les relations entre utilisateurs et fournisseurs, les relations entre firmes, les organes réglementaires et les instituts de recherche, et les stimulants qui s'exercent au sein de "grappes" de concurrents, sont autant de facteurs qui génèrent des courants d'information propices à l'innovation ou qui conduisent les entreprises à se montrer plus réceptives ;

- l'innovation au sein d'une firme peut dépendre de manière cruciale de la présence de spécialistes qui "filtrent" l'information ou qui la reçoivent - les personnes qui, par des moyens multiples et divers, assurent une veille technologique en se tenant au courant des évolutions nouvelles (y compris la technologie nouvelle et le savoir codifié contenu dans les brevets, la presse spécialisée et les revues scientifiques) et qui entretiennent des réseaux personnels facilitant les courants d'informations ;

- les liens internationaux sont un volet essentiel des réseaux à travers lesquels l'information est canalisée - les réseaux ("collèges invisibles") d'experts internationaux sont l'un des principaux moyens de transmettre des connaissances scientifiques actualisées et des évolutions technologiques de pointe ; 
- le degré de mobilité des ingénieurs ou scientifiques influera sur la rapidité avec laquelle les développements nouveaux peuvent se répandre ;

- la commodité d'accès de l'industrie aux capacités de la $\boldsymbol{R}$-D publique ;

- la constitution de sociétés ad hoc - qui suppose habituellement le transfert d'individus particulièrement compétents - est souvent un moyen précieux de commercialiser des développements nouveaux issus de la recherche menée dans le secteur public ;

- l'éthique, les systèmes de valeurs collectives, la confiance et l'ouverture d'esprit sont des facteurs qui influencent le degré d'efficacité des réseaux, relations et autres voies de communication, en ce qu'ils affectent les rapports informels entre individus qui sont la pierre angulaire de multiples accords commerciaux, et en ce qu'ils déterminent les paramètres et les règles de comportement acceptées dans le cadre desquels interviennent la communication et les échanges d'informations; et

- le savoir codifié contenu dans les brevets, la presse spécialisée et les revues scientifiques.

\subsection{La "dynamo de l'innovation"}

84. Par "dynamo de l'innovation", on entend le réseau complexe de facteurs qui façonne l'innovation au niveau de l'entreprise. Placer la dynamo de l'innovation au centre de la carte, c'est reconnaître que la capacité d'innovation d'une économie dépend pour beaucoup de l'entreprise. Il importe donc de comprendre les caractéristiques qui rendent les firmes plus ou moins innovantes, ainsi que la manière dont l'innovation se crée à l'intérieur des entreprises. La propension d'une firme à innover dépend bien évidemment des ouvertures technologiques qui s'offrent à elle. De plus, les firmes n'ont pas toutes la même aptitude à déceler et à exploiter les ouvertures technologiques. Pour innover, une firme doit se faire une idée de la nature de ces ouvertures, élaborer une stratégie en conséquence et avoir les moyens de transformer ces inputs en une innovation effective - et de le faire plus rapidement que ses concurrents. Mais on ne saurait s'arrêter là. Nombre d'ouvertures technologiques ne sont pas le fruit d'une génération spontanée: elles sont conçues par des entreprises qui veulent atteindre certains objectifs stratégiques (par exemple, satisfaire une demande identifiée du marché). La capacité d'innovation réside dans un ensemble de facteurs, que l'entreprise possède ou non, et dans la manière d'associer ces facteurs de manière efficace.

85. La capacité technologique d'une entreprise repose en partie sur son personnel. Avoir des employés compétents est un atout majeur pour une entreprise innovante. Une firme qui ne possède pas de travailleurs compétents est dans l'incapacité de maîtriser les technologies nouvelles et, a fortiori, d'innover. En plus des chercheurs, elle a besoin d'ingénieurs capables de gérer les opérations de production, de vendeurs à même de comprendre la technologie qu'ils proposent à la vente (il s'agit à la fois pour eux de la vendre et de communiquer en retour les suggestions des acheteurs), ainsi que de gestionnaires avertis des questions technologiques.

86. La capacité d'innover est également fonction des caractéristiques de la firme : la structure de son personnel et de ses installations (compétences, départements), sa structure financière, sa stratégie commerciale, ses concurrents, les alliances nouées avec d'autres entreprises ou avec des universités et, notamment, son organisation interne. Beaucoup de ces aspects sont complémentaires. Un type particulier de structures de compétences va de pair avec un type donné de stratégie, de structure financière, etc. 
87. Les options qui s'offrent à une entreprise désireuse d'innover, c'est-à-dire de modifier ses atouts et moyens technologiques, ainsi que ses résultats au niveau de la production, sont de trois ordres : activités stratégiques, activités de R-D et activités autres que la R-D.

- Activités stratégiques : avant de s'engager dans des activités d'innovation, les entreprises doivent prendre des décisions - explicites ou implicites - sur les types de marchés qu'elles desservent, ou qu'elles cherchent à créer, et les types d'innovations qu'elles vont tenter d'introduire sur ces marchés.

- R-D : certaines des options concernent la R-D (telle qu'elle est définie dans le manuel de Frascati, y compris le développement expérimental qui va bien au-delà de la recherche fondamentale et de la recherche appliquée) :

i) l'entreprise peut entreprendre des travaux de recherche fondamentale pour mieux connaître les processus fondamentaux liés à ce qu'elle fabrique ;

ii) elle peut se lancer dans la recherche stratégique (c'est-à-dire intéressant l'industrie mais sans applications précises) pour élargir l'éventail des projets concrets que cela lui ouvre, et dans la recherche appliquée en vue de parvenir à des inventions particulières ou à des modifications des techniques existantes ;

iii) elle peut développer des concepts de produit afin de déterminer s'ils sont réalisables et viables, stade qui implique (a) de concevoir un prototype, (b) de le développer et de le tester, et (c) de poursuivre les recherches en vue de modifier les plans ou les fonctions techniques.

- Activités autres que la R-D : l'entreprise peut entreprendre bon nombre d'autres activités qui n'ont pas de lien direct avec la R-D, et ne sont pas définies comme R-D, bien qu'elles jouent un rôle important dans l'innovation et l'efficacité de l'entreprise :

iv) elle peut définir de nouveaux concepts de produit et de nouvelles technologies de production par l'intermédiaire : (a) de son service de commercialisation et de ses relations avec les utilisateurs, (b) de l'identification de possibilités de commercialisation provenant de ses propres recherches fondamentales ou stratégiques ou de celles des autres ; $(c)$ de ses moyens de conception et de ses capacités d'ingénierie, $(d)$ de la surveillance des concurrents; et (e) du recours à des experts-conseils ;

v) elle peut mettre en place des installations pilotes, puis des installations de production en vraie grandeur ;

vi) elle peut acheter des informations techniques, en payant des droits ou des redevances pour les inventions brevetées (dont l'adaptation et la modification exigent habituellement des travaux de recherche et d'ingénierie) ou acquérir un savoir-faire et des compétences en ayant recours aux services d'ingénieurs-conseils ou d'experts-conseils de divers types ;

vii) elle peut développer les qualifications du personnel de fabrication (par une formation en entreprise) ou les acquérir (en recrutant) ; cela peut impliquer aussi un apprentissage tacite et informel du type "initiation pratique au travail"; 
viii) elle peut investir dans l'équipement de transformation ou d'autres facteurs intermédiaires de production, qui incorporent le fruit du travail d'innovation d'autres entreprises ; il peut s'agir en l'occurrence de composants, de machines, voire d'une usine complète.

ix) elle peut réorganiser ses systèmes de gestion et l'ensemble de son système de production ainsi que ses méthodes, avec notamment de nouveaux modes de gestion des stocks et de contrôle de qualité, et une amélioration constante de la qualité.

88. De multiples tentatives ont été faites pour construire des modèles pour élucider la manière dont l'innovation est générée à l'intérieur des entreprises, en prenant en compte l'influence exercée par des événements extérieurs aux entreprises. Le modèle dit de "liaison en chaîne" de Kline et Rosenberg" 12 est précieux à cet égard (figure 2).

Figure 2. Modèle dit de "liaison en chaîne" du processus d'innovation"

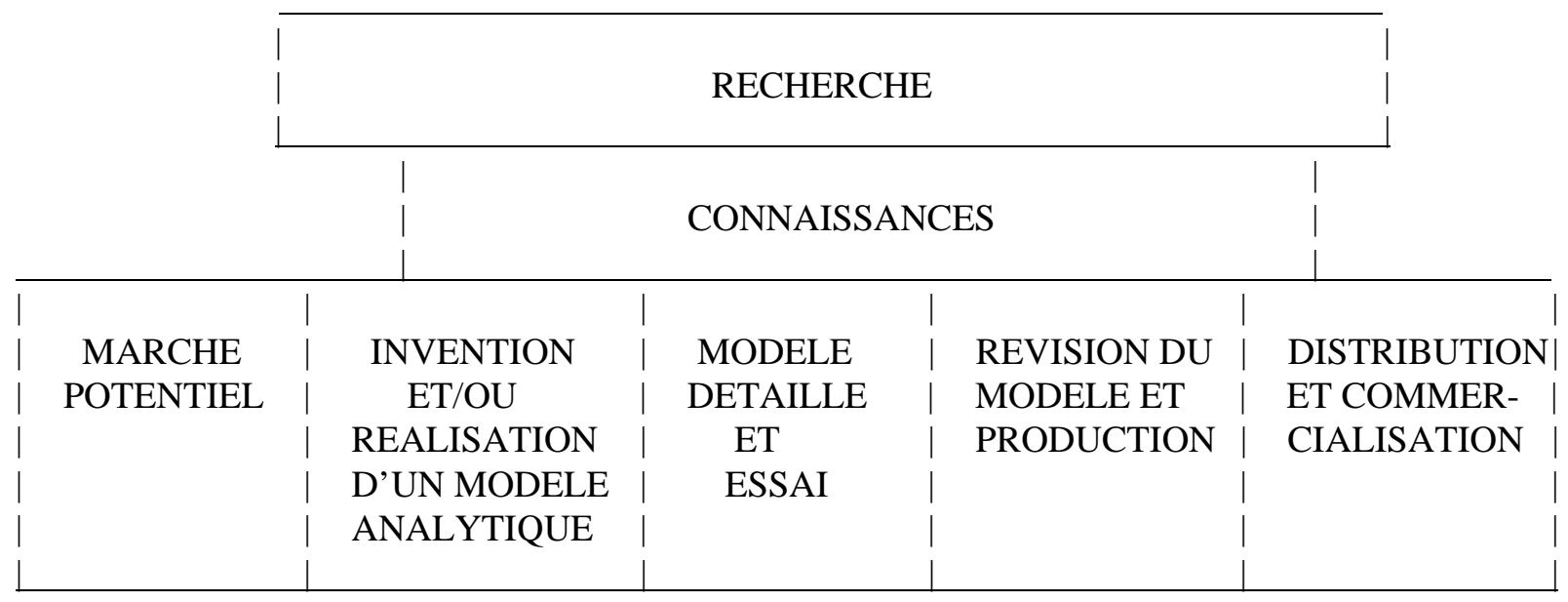

89. Dans le modèle de liaison en chaine, l'innovation est conçue comme une interaction entre, d'une part, les possibilités offertes par le marché et, d'autre part, la base de connaissances et les moyens dont dispose l'entreprise. Chacune de ces fonctions se subdivise en un certain nombre de sous-processus, dont l'issue est souvent très incertaine. En conséquence, il n'y a pas de progression simple, et il faut souvent revenir à des phases antérieures pour surmonter des difficultés de mise au point. Cela implique l'existence d'une rétroaction entre toutes les parties du processus. L'aptitude des firmes à maintenir des liaisons efficaces entre les phases du processus d'innovation est un élément déterminant du succès (ou de l'échec) d'un projet d'innovation: le modèle souligne, par exemple, l'importance cruciale d'une interaction continue entre la phase de commercialisation et celles d'invention/conception ${ }^{14}$.

90. Quel est le rôle de la recherche dans l'innovation ? Dans le modèle de liaison en chaîne la recherche est considérée non pas comme une source d'idées novatrices mais comme un moyen de résoudre les problèmes à laquelle on peut recourir à n'importe quel stade. Lorsque des problèmes surgissent dans le processus d'innovation, ce qui ne saurait manquer d'arriver, une firme puise dans sa base de connaissances à ce moment particulier, base qui est constituée par les résultats de recherches antérieures ainsi que par l'expérience technique et pratique. Le système de recherche revient sur les difficultés qui n'ont pas pu être réglées à l'aide de la base de connaissances existantes, qu'il enrichit ainsi en cas de succès. 
91. Cette façon de voir a des répercussions sur la manière dont on envisage la "recherche". Dans la mesure où celle-ci peut se rapporter à n'importe quelle phase du processus d'innovation, la recherche est une activité complexe et différenciée au plan interne, comportant en puissance une grande variété de fonctions. Elle vient compléter l'innovation et n'en constitue pas une condition préalable. En fait, de nombreuses activités de recherche seront modelées par le processus d'innovation et bien des problèmes à traiter découleront d'idées novatrices qui sont générées ailleurs. C'est pourquoi, dans la démarche à liaisons en chaîne, la recherche ne peut pas être considérée uniquement comme le travail de découverte qui précède l'innovation.

92. Rothwell ${ }^{15}$ a examiné un certain nombre d'autres méthodes de modélisation, notamment l'élaboration de modèles parallèles fondés sur des degrés élevés d'intégration fonctionnelle. Il suggère de les étendre à des modèles de la "cinquième génération" ou modèles SIN (intégration des systèmes et constitution de réseaux) qui prennent également en compte les modifications intervenant dans les technologies à travers lesquelles le changement technologique est lui-même transmis.

93. Il ne s'agit pas, dans la présente analyse, de présenter un modèle particulier d'innovation en prétendant qu'il est définitif. Tous les modèles existants suscitent certaines interrogations sérieuses. Il convient toutefois de noter que l'innovation est une activité complexe, diversifiée, comportant de nombreux composants en interaction, ce que doivent refléter les sources de données.

\section{CHOIX DES PRIORITÉS}

94. Dans le monde entier, les gouvernements sont aujourd'hui confrontés à toute une série de problèmes touchant l'élaboration d'indicateurs qui se rapportent à l'économie fondée sur le savoir. Les ministres de la science et de la technologie des pays de l'OCDE ont souligné la nécessité de perfectionner notablement les indicateurs touchant ce domaine ${ }^{16}$ :

“... le développement rapide de l'ensemble des recherches sur l'innovation, y compris la nouvelle théorie de la croissance, a des implications importantes pour l'évolution des politiques scientifiques et technologiques, ainsi que pour les autres politiques affectant les performances nationales en matière d'innovation... Les ministres ont convenu qu'il est nécessaire pour les pays Membres de collaborer au développement d'une nouvelle génération d'indicateurs pouvant mesurer les performances en matière d'innovation d'une économie fondée sur le savoir, et les autres "produits" qui y sont liés."

95. Le Livre vert de la Commission européenne formule également des recommandations :

“... des enquêtes statistiques régulières sur l'innovation technologique devraient être organisées dans les États membres. Ces enquêtes doivent permettre de mesurer, entre autres, les coûts et les bénéfices résultant des activités innovantes et de mieux comprendre les déterminants de l'innovation."

96. Il est essentiel de nourrir le débat sur les questions de politique avec des informations et des analyses relatives à de multiples aspects de l'innovation. Dans l'idéal, il faudrait mettre en place un système d'information d'ensemble couvrant tous les types de facteurs qui interviennent dans le terrain politique de l'innovation, comme présenté plus haut. Les gouvernements seraient ainsi solidement armés pour faire face comme il convient à n'importe quelle question qui se poserait à eux. Dans la pratique, certaines parties seulement d'un système de ce genre peuvent être couvertes par des indicateurs, tandis que d'autres exigent des informations qualitatives. Qui plus est, comme le savent pertinemment les analystes des politiques et des indicateurs, ce n'est qu'occasionnellement que les indicateurs se rattachent nettement à une question ou à un facteur unique et le plus souvent ils se rapportent à toute une série de 
questions en ne couvrant qu'une partie seulement de chacune d'elles. Tout vaste système d'information ou de suivi devra également être complété par des études de cas lorsque le besoin d'une analyse ponctuelle approfondie se fait sentir. Par ailleurs, il importe aussi de prendre en considération, dans toute la mesure du possible, une série d'indicateurs et d'autres informations - même si l'attention se porte directement sur un sujet très particulier ou sur une gamme relativement étroite de thèmes.

\subsection{Six domaines d'étude}

\subsubsection{Stratégies des firmes}

97. Il n'est guère facile de classer les stratégies technologiques au moyen d'une enquête. Il est néanmoins possible de demander aux firmes comment elles perçoivent le développement de leurs marchés et l'importance des divers choix stratégiques concernant le développement des produits et des marchés. Etant donné que les schémas particuliers de combinaison ont leur importance du point de vue de l'action des pouvoirs publics, aucun effort ne doit être ménagé pour obtenir des données classées par type de stratégie.

\subsubsection{Rôle de la diffusion}

98. Il convient de ne pas négliger l'importance de la diffusion. Une innovation restera sans grand effet si elle n'est pas appliquée très largement au-delà de son lieu d'origine (pour la première fois dans le monde) dans d'autres pays, d'autres industries et même dans des entreprises appartenant à la même branche d'activité.

99. Un problème que soulève souvent l'analyse du progrès technologique et de la croissance de la productivité tient au fait qu'il est extrêmement difficile de suivre les flux d'innovation et de progrès technologique d'une industrie à l'autre et, par conséquent, de déceler les "retombées" d'une activité visant à accroître la productivité ${ }^{17}$. Comment les entreprises intègrent-elles les innovations qui ont été mises au point ailleurs? Et quel est le poids de la diffusion par rapport à l'innovation ?

100. Un objectif à assigner aux travaux d'enquête complémentaires serait de préciser la nature de ces flux entre industries. Le fait de se polariser uniquement sur les innovations générées à l'intérieur des firmes donne une idée trompeuse de l'impact économique de l'innovation sur le progrès technologique. D'une manière ou d'une autre, il faut faire une distinction entre les sources/destinations internes et externes des résultats des activités d'innovation.

101. Une question distincte, mais connexe, concerne le rôle de la coopération interentreprise sous forme de R-D partagée, d'octroi de licences, d'entreprises conjointes, etc. Dans de nombreux secteurs industriels, les arrangements de coopération se sont généralisés à un point tel qu'il est difficile de distinguer les processus d'innovation les uns des autres, et parfois même de situer les limites de la firme.

102. Les répercussions de toutes ces questions au plan de l'action gouvernementale sont évidentes puisque celle-ci vise en général, explicitement ou implicitement, à promouvoir la R-D et s'intéresse beaucoup moins aux autres éléments du processus d'innovation. En particulier, on privilégie souvent la capacité d'invention par rapport à la capacité d'adoption de la technologie alors même que cette dernière est un facteur clé de l'efficacité d'une firme. 


\subsubsection{Sources d'information pour l'innovation et obstacles à l'innovation}

103. L'objectif global en l'occurrence devrait être d'établir un lien entre, d'une part, les atouts et les stratégies technologiques des firmes et, d'autre part, la portée de leurs sources d'information pour l'innovation et les obstacles qu'elles perçoivent. La plupart des firmes disposent d'un large éventail de sources potentielles d'informations techniques. Leur importance varie selon les moyens et la stratégie technologiques de la firme.

104. Il importe d'établir une distinction entre les sources de changement internes et externes (c'est-à-dire endogènes et exogènes). A l'intérieur de la firme, l'intérêt se portera vraisemblablement surtout sur le rôle - ou les rôles - du service de R-D, ainsi que sur la participation aux décisions d'innover et aux activités d'innovation de tous les secteurs de la firme, en particulier des responsables de la commercialisation. A l'extérieur, l'attention se portera surtout sur les établissements publics de recherche en tant que sources d'informations techniques, et sur les flux technologiques interentreprises ou interindustries. La prise en considération des sources externes de l'innovation ou du progrès technologique devrait logiquement aller jusqu'aux sources internationales de technologie et être structurée de manière à apporter des éléments de réponse à quelques-uns des nombreux problèmes non résolus se rapportant à la balance des paiements technologiques.

105. Un problème à régler à ce stade est celui de la classification des entreprises et des industries qui est utilisée pour l'analyse des flux de technologies. Pavitt parle d'“entreprises dominées par les fournisseurs", d' "entreprises à forte intensité productive" et d'“entreprises fondées sur la science", et il se sert de la base de données SPRU pour analyser les rapports entre ces catégories. Archibugi et ses collègues emploient une classification analogue pour analyser les données italiennes ${ }^{18}$.

106. Le problème sous-jacent, fort important du point de vue de l'action gouvernementale, est que nous connaissons assez mal les facteurs liés à l'environnement, aux circonstances ou à la réglementation qui déterminent la localisation de l'innovation ${ }^{19}$ dans les réseaux complexes d'entreprises où, à l'évidence, l'innovation peut survenir dans toute une variété de lieux.

107. Les obstacles à l'innovation revêtent aussi de l'importance pour l'action des pouvoirs publics puisqu'une bonne part des mesures gouvernementales visent à les surmonter d'une manière ou d'une autre. De nombreux obstacles - manque de personnel qualifié, problèmes de compétence, financement, possibilité d'appropriation - sont relativement simples à déterminer grâce aux méthodes d'enquête.

\subsubsection{Inputs pour l'innovation}

108. Comme point de départ pour l'analyse de l'innovation, on pourrait prendre la R-D, qui revêt une grande diversité de formes fonctionnelles liées à la solution de problèmes. Ainsi, on prétend souvent que les firmes ont besoin de procéder à des travaux de R-D pour connaître et utiliser, et par conséquent pour adopter, des technologies mises au point ailleurs. ${ }^{20}$

109. Bien qu'il soit souhaitable d'inclure dans l'enquête une mesure de la R-D, la tâche essentielle est d'intégrer au processus d'innovation une explication de la contribution de la R-D avec une prise en compte des inputs non R-D. Ces derniers ont été décrits précédemment dans ce chapitre. Il serait extrêmement utile d'avoir un aperçu de la place relative que les firmes accordent aux activités de R-D et aux activités non R-D, ainsi que de leur répartition dans certaines industries et dans l'ensemble des industries. Une meilleure connaissance de ces répartitions et de leurs variations selon les secteurs revêt une importance manifeste pour la politique de l'innovation ${ }^{21}$. Ces éléments pourraient aussi faciliter la prise des décisions relatives à l'équilibre souhaitable des mesures gouvernementales concernant les 
aspects R-D et non R-D de l'innovation. Si la collecte de ces informations peut soulever de grandes difficultés d'ordre pratique, surtout lorsque les firmes comportent de nombreux services, elle constitue néanmoins l'une des perspectives les plus intéressantes de ce genre de travaux d'enquête ${ }^{22}$.

\subsubsection{Rôle des pouvoirs publics dans l'innovation industrielle}

110. Etant donné que la R-D financée sur fonds publics représente souvent une part notable de l'ensemble de la R-D dans les économies de l'OCDE, il est à l'évidence nécessaire d'avoir une idée précise de ses effets sur l'industrie. Mais la R-D n'est qu'un élément de l'action gouvernementale qui influe sur l'efficacité en matière d'innovation.

111. D'autres aspects peuvent aussi favoriser cette efficacité ou la limiter (l'enseignement et l'offre de personnel qualifié; la politique fiscale et la réglementation en matière de comptabilité; la réglementation industrielle concernant tout particulièrement l'environnement, les normes de santé, les contrôles de qualité, la normalisation, etc.; le régime juridique applicable aux droits de propriété intellectuelle et, partant, les problèmes d'appropriation ainsi que le fonctionnement des systèmes de brevets et de droits d'auteur; le fonctionnement du marché des capitaux). Ces aspects de l'action des pouvoirs publics peuvent être étudiés au moyen de questions relatives à la manière dont les firmes perçoivent les obstacles à l'innovation.

112. Dans le cas des données sur la R-D, on peut utilement chercher à déterminer dans quelle mesure les applications industrielles peuvent dépendre des résultats des travaux de recherche fondamentale menés par les universités et des laboratoires financés sur fonds publics ${ }^{23}$.

\subsubsection{Outputs de l'innovation}

113. L'un des aspects les plus intéressants, peut-être, de ces enquêtes tient à ce qu'elles sont susceptibles de permettre une mesure directe de l'output des activités d'innovation. Il ressort des enquêtes effectuées dans le passé qu'une proportion très élevée de firmes avait introduit des innovations au cours de l'année précédente, ce qui montre que l'activité d'innovation est beaucoup plus largement répandue que les données sur la R-D ne le laisseraient supposer, car la R-D est extrêmement concentrée sur le plan aussi bien industriel que géographique ${ }^{24}$. Mais définir en quoi consiste une innovation ne va pas sans poser de multiples problèmes. La plupart des produits, et certainement les procédés utilisés pour les fabriquer, sont des ensembles complexes. Il faut donc définir le changement par rapport :

- aux attributs et aux caractéristiques de performance du produit considéré dans son ensemble ; et

- aux modifications des composants du produit, qui en améliorent l'efficacité, y compris la nature des services qu'il rend. Les modifications de sous-systèmes de ce type peuvent être d'une portée très limitée mais leur incidence cumulative peut être considérable et avoir son importance dans une perspective analytique.

114. Le chapitre 3 aborde diverses questions se rapportant à ces problèmes de définition, notamment : la distinction à faire entre un produit nouveau et un produit technologiquement amélioré (les perfectionnements esthétiques ou les perfectionnement techniques mineurs sont considérés non pas comme une innovation mais comme une différenciation de produit); le sens à donner au mot "nouveau"; la comparaison des définitions du type de nouveauté utilisées par les firmes, afin de faciliter le traitement des questions se rapportant à la proportion de ventes ou d'exportations qui est imputable à ces produits nouveaux ou améliorés. 
115. La très grande diversité des processus d'innovation, sous l'angle de leurs objectifs, de leur organisation, de leur coût, de l'utilisation de la recherche, etc., se traduit aussi par toute une variété de problèmes et contraintes que les firmes doivent surmonter pour introduire avec succès un changement technologique. Il y a donc lieu de faire une distinction entre les aspects du processus d'innovation qui peuvent être mesurés et ceux qui ne le peuvent pas, ainsi que de clarifier les relations entre la méthode de mesure et le processus qui la sous-tend.

116. Les enquêtes sur l'innovation du type esquissé dans ce manuel fournissent principalement des informations sur la dynamo de l'innovation et les facteurs de transferts environnants (voir la figure 1 cidessus), qui mettent l'accent sur l'importance de la firme pour l'innovation. Le fait de mettre la firme au coeur de l'enquête exerce sur le champ des mesures, un certain nombre de conséquences qui seront brièvement examinées ci-après :

- que voulons-nous mesurer?

- comment voulons-nous procéder?

- où voulons-nous effectuer les mesures ?

4.2.1 "Que voulons-nous mesurer ?": Les innovations technologiques de produit et de procédé Innovations TPP

117. Ce manuel est consacré à l'innovation au niveau de la firme. Lorsque des firmes innovent, elles s'engagent dans un ensemble complexe d'activités aux conséquences multiples, dont quelques-unes de surcroît peuvent remodeler les limites et la nature de la firme elle-même. Il faut donc prendre une décision sur celles de ces activités et conséquences que l'on devrait et que l'on peut mesurer.

118. Le manuel est centré sur deux catégories définies par Schumpeter, les produits et procédés nouveaux et améliorés, qui doivent répondre au minimum au critère "nouveau pour la firme" afin de répondre aux recommandations relatives à la diffusion. Pourtant, l'expérience pratique l'a montré ; les changements apportés aux produits (et, dans une bien moindre mesure, aux procédés) que les firmes jugent nouveaux ou améliorés ne cadrent pas tous avec le modèle de changement technique précédemment décrit. Il ne suffit donc pas d'exclure les changements insignifiants, mineurs ou ne comportant pas un degré significatif de nouveauté : en effet, il faut aussi prendre une décision sur la manière de traiter les changements d'ordre esthétique qui peuvent rendre les produits beaucoup plus séduisants pour les acheteurs et peuvent donc exercer un effet important sur les performances de la firme en cause. Le présent manuel porte uniquement sur l'innovation "technologique", qui suppose impérativement une amélioration objective des performances d'un produit.

119. Pour mettre en chantier une innovation, les firmes doivent modifier, d'une manière ou d'une autre, leur stock de biens matériels et immatériels. Dans les biens immatériels peuvent entrer les capacités et compétences acquises tout au long d'un processus d'apprentissage. Etant donné que l'innovation comporte de multiples facettes, l'un de ses éléments déterminants réside dans l'organisation, aspect qui a polarisé l'attention ces dernières années. L'organisation se ramène en substance à une série de processus par lesquels s'effectuent la collecte, la gestion et l'exploitation de l'information, ainsi que la mise en oeuvre des décisions fondées sur cette information. Ces processus sont en grande part immatériels mais, pris ensemble, ils constituent la capacité d'apprentissage de la firme et, en tant que tels, ils sont un élément central de la capacité d'innovation. Il s'agit là de "règles du jeu" spécifiquement institutionnelles 
qui déterminent les modalités possibles d'organisation sur un large plan mais, à l'intérieur de ces paramètres institutionnels, les firmes peuvent présenter une grande diversité, ce qui se vérifie en fait. Si l'on examine les firmes sous l'angle de la théorie de l'information et de l'apprentissage, il paraît évident que l' "organisation" n'a peut-être pas grand chose à voir avec la notion de structures formelles.

120. De ce point de vue, l'organisation est une dimension critique de l'innovation mais, tant théoriquement qu'en pratique, sa mesure apparaît comme très délicate. Qui plus est, étant donné que le changement organisationnel est très spécifique d'une firme, il est plus difficile encore de le résumer dans des statistiques globales, sectorielles ou établies à l'échelle de l'ensemble de l'économie. Par conséquent, l'innovation organisationnelle ne fait pas partie des mesures recommandées dans le corps du manuel.

121. Une approche statistique peut être féconde dans ce domaine mais, à bien des égards, des études de cas peuvent suffire à éclairer quelques-unes des caractéristiques importantes du changement organisationnel. On trouvera à l'annexe 2 de plus amples détails sur la collecte de données se rapportant à l'innovation non technologique.

\subsection{2 “Comment voulons-nous procéder?” : choix de l'approche pour l'enquête}

122. Il existe deux grandes méthodes de collecte de données sur l'innovation :

- 1'"approche sujet" part des attitudes et des activités innovantes de la firme dans son ensemble. Il s'agit de déterminer les facteurs qui influent sur le comportement novateur de la firme (stratégies, incitations et obstacles à l'innovation) et le champ des diverses activités d'innovation, et, surtout, de se faire une idée des outputs et des effets de l'innovation. Les enquêtes de ce genre sont conçues de manière à être représentatives de chaque industrie dans son ensemble de sorte que, après extrapolation des résultats bruts, il est possible de procéder à des comparaisons entre les industries ;

- La seconde démarche consiste à réunir des données sur des innovations spécifiques (généralement une 'innovation significative' d'une sorte ou d'une autre, ou la principale innovation d'une firme) : il s'agit de 1' "approche objet". On commence par dresser une liste des innovations couronnées de succès, en se fondant souvent sur des évaluations d'experts ou une liste des nouveaux produits annoncés dans les revues spécialisées. L'objectif est de rassembler un certain nombre de données descriptives, quantitatives et qualitatives au sujet d'une innovation donnée en même temps que des données sont recherchées à propos de la firme.

123. Du point de vue du développement économique en cours, ce sont les degrés différents de réussite des firmes qui déterminent les conséquences économiques et donc qui présentent un intérêt pour l'action des pouvoirs publics. Ce qui compte, c'est le sujet, c'est-à-dire les firmes, et on a choisi de fonder ces principes directeurs sur la première méthode.

124. De plus, l'approche "sujet" se prête mieux à une normalisation internationale. Les définitions et classifications proposées sont donc formulées en vue essentiellement de leur utilisation lors de la conception d'enquêtes sur l'innovation des firmes, mais on a pris garde à ce qu'elles puissent aussi être utiles pour d'autres types d'enquêtes sur l'innovation. L'approche "objet" (y compris les enquêtes fondées sur la presse spécialisée) est décrite plus en détail à l'annexe 1. 


\subsection{3 "Où voulons-nous effectuer les mesures?” : couverture sectorielle}

125. L'innovation peut, bien entendu, intervenir dans n'importe quel secteur de l'économie, y compris dans les services publics, par exemple dans le domaine de la santé ou dans celui de l'éducation. Etant donné l'accent mis sur la firme, les concepts et définitions qui seront présentés dans ce manuel sont principalement conçus pour s'appliquer aux innovations intervenant dans le secteur des entreprises.

126. La version précédente du manuel portait uniquement sur l'innovation dans l'industrie manufacturière. Depuis lors, le secteur des services est devenu le centre de gravité des questions touchant l'emploi et la production, d'où la nécessité de mieux comprendre ses activités technologiques. Il apparaît d'ores et déjà à l'évidence que les services sont les principaux utilisateurs de l'innovation générée dans le secteur manufacturier (OCDE : 1995). Certaines enquêtes récentes sur la R-D donnent à penser que le secteur des services joue un rôle important dans la création du savoir (OCDE : 1996). Dans nombre de domaines, la frontière entre l'industrie et les services en tant que secteurs innovants est en train de s'estomper (c'est ainsi que les logiciels représentent une part grandissante de la plupart des innovations annoncées comme venant de l'industrie). Il y a donc lieu d'étendre les enquêtes sur l'innovation au secteur des services.

127. La tâche n'est pas aisée pour quatre raisons :

- l'innovation n'a pas les mêmes caractéristiques dans le secteur des services que dans le secteur manufacturier. Dans le secteur des services, l'innovation est souvent immatérielle et, partant, difficile à protéger. Les services sont plus personnalisés. Il existe une interrelation plus étroite entre la création de nouveaux services et les procédés permettant de les produire ;

- le contexte statistique est également différent. Des programmes statistiques bien établis existent pour les services consistant dans la manutention de biens, y compris le commerce de gros et de détail, le fret et le transport. Dans ces branches d'activité, on dispose ainsi de solides mesures de la production, de l'investissement, des prix et des activités financières : il est donc plus facile de distinguer les différences qui séparent les innovateurs de ceux qui ne le sont pas, ainsi que d'en déduire des conséquences pour l'action gouvernementale. Les statistiques dont on peut disposer comme contexte de référence à la mesure de l'innovation sont plus incertaines quand il s'agit de branches d'activité économique qui ne sont pas directement liées à la manutention de biens et certains de ces services sont économiquement importants tout en étant des instruments du changement technologique et social. Il s'agit d'industries de service intéressant les communications, la finance, l'assurance et l'immobilier, les loisirs et les services aux entreprises ;

- d'une manière générale, les firmes dans les industries de service sont plus petites que celles de l'industrie manufacturière et moins concentrées aussi. Ce constat a des conséquences méthodologiques pour la réalisation des enquêtes par sondage et pour les estimations ;

- les industries de service ne sont pas toutes semblables. Elles diffèrent les unes des autres sous l'angle des compétences requises, de l'organisation des fonctions de production et de commercialisation, des niveaux de technologie utilisés et des marchés desservis. Leurs propensions à participer au commerce international et à innover sont probablement différentes et elles peuvent réagir différemment aux conditions économiques.

128. On a néanmoins adapté les définitions et concepts utilisés dans ce manuel en se fondant sur l'expérience acquise jusqu'ici, de manière à ce qu'elles s'appliquent aux innovations TPP dans les 
industries manufacturières, la construction, la production et distribution de l'électricité, gaz et eau, ainsi que les services commercialisés. 


\section{Chapitre 3}

\section{DÉFINITIONS DE BASE}

\section{INTRODUCTION}

129. Le présent chapitre, qui constitue la première étape vers l'établissement d'un cadre statistique à partir des concepts et des priorités définis au chapitre 2 , décrit le phénomène au sujet duquel des informations pourront être recueillies sur des bases comparables à l'échelle internationale. Il a pour objet de présenter un ensemble de définitions cohérentes et aussi précises que possible des différents types d'innovations, des activités d'innovation et, partant, de firmes innovantes. Il n'est pas toujours possible de donner des définitions très précises en raison de la complexité du processus d'innovation et du fait qu'il intervient de différentes manières selon le type de firme ou de branche d'activité qui le met en oeuvre ; il faut donc adopter des conventions en la matière. Chaque fois qu'il en existe la possibilité, des exemples sont présentés afin d'illustrer les différentes catégories et les distinctions qu'il est recommandé de faire entre elles.

\section{INNOVATION TPP}

130. Les innovations technologiques de produit et de procédé (TPP) couvrent les produits et procédés technologiquement nouveaux ainsi que les améliorations technologiques importantes de produits et de procédés qui ont été accomplis. Une innovation TPP a été accomplie dès lors qu'elle a été introduite sur le marché (innovation de produit) ou utilisée dans un procédé de production (innovation de procédé). Les innovations TPP font intervenir toutes sortes d'activités scientifiques, technologiques, organisationnelles, financières et commerciales. La firme innovante TPP est une firme qui a accompli des produits ou des procédés technologiquement nouveaux ou sensiblement améliorée au cours de la période considérée.

131. Le niveau minimal d'entrée correspond au fait que le produit ou le procédé soit nouveau (ou sensiblement amélioré) pour la firme (il ne doit pas nécessairement être nouveau à l'échelle mondiale).

132. Les innovations TPP concernant l'activité primaire et secondaire sont prises en compte de même que les innovations de procédé dans les activités auxiliaires.

\section{PRINCIPAUX ÉLÉMENTS DE L'INNOVATION TPP}

133. Les innovations TPP peuvent être ventilées en produit et procédé, et en fonction du degré de nouveauté du changement introduit dans chaque cas. 


\subsection{Innovations technologiques de produit}

134. Le terme "produit" est utilisé pour désigner à la fois les biens et les services.

Ceci est conforme au Système de comptabilité national (CE et al. 1993).

135. L'innovation technologique de produit peut prendre deux grandes formes :

- des produits technologiquement nouveaux ${ }^{25}$;

- des produits technologiquement améliorés ${ }^{26}$.

136. Un produit technologiquement nouveau est un produit dont les caractéristiques technologiques ou les utilisations prévues présentent des différences significatives par rapport à ceux produits antérieurement. De telles innovations peuvent faire intervenir des technologies radicalement nouvelles, ou reposer sur l'association de technologies existantes dans de nouvelles applications, ou découler de la mise à profit de nouvelles connaissances.

137. Les premiers microprocesseurs ou magnétoscopes étaient des exemples de produits technologiquement nouveaux, du premier type, faisant appel à des technologies radicalement nouvelles. Les premiers baladeurs, qui associaient les techniques existantes de la bande magnétique et des miniécouteurs, représentaient un produit technologiquement nouveau du deuxième type, conjuguant des technologies existantes dans une nouvelle application. Dans les deux cas, le produit final n'existait pas antérieurement.

138. Un produit technologiquement amélioré est un produit existant dont les performances sont sensiblement augmentées ou améliorées. Un produit simple peut être amélioré (par amélioration des performances ou abaissement du coût) grâce à l'utilisation de composants ou de matériaux plus performants, ou bien un produit complexe, qui comprend plusieurs sous-systèmes techniques intégrés, peut être amélioré au moyen de modifications partielles apportées à l'un des sous-systèmes.

139. Les produits technologiquement améliorés peuvent avoir des conséquences d'importance aussi bien majeure que mineure pour la firme. Le remplacement du métal par des matières plastiques dans l'équipement ou le mobilier de cuisine est un exemple d'utilisation de composants offrant de meilleures performances. L'introduction du freinage ABS ou d'autres améliorations de sous-systèmes sur les automobiles est un exemple de modification partielle d'un sous-système technique intégré parmi d'autres.

140. La distinction entre un produit technologiquement nouveau et un produit technologiquement amélioré peut poser des difficultés dans certaines branches d'activité, notamment dans le secteur des services. 


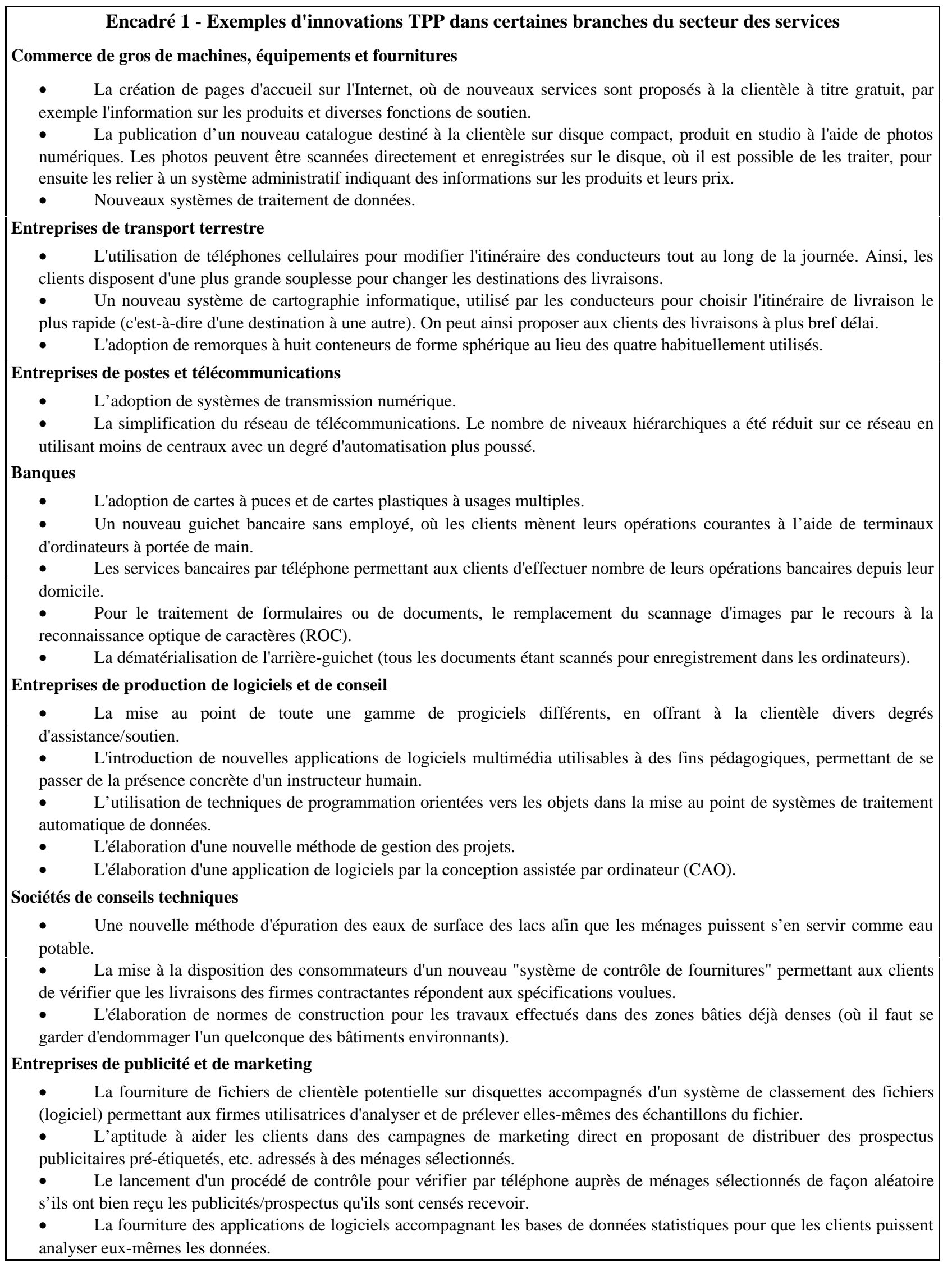




\subsection{Innovation technologique de procédé}

141. Il y a innovation technologique de procédé dans le cas de l'adoption de méthodes de production technologiquement nouvelles ou sensiblement améliorées, y compris les méthodes de livraison du produit. Ces méthodes peuvent impliquer des modifications portant sur l'équipement ou l'organisation de la production, ou une combinaison de ces modifications, et peut découler de la mise à profit de nouvelles connaissances. Ces méthodes peuvent viser à produire ou à livrer des produits technologiquement nouveaux ou améliorés, qu'il est impossible de produire ou de livrer à l'aide de méthodes classiques, ou essentiellement à augmenter le rendement de production ou l'efficacité de la livraison de produits existants.

142. Dans certaines branches du secteur des services, il n'est pas toujours facile d'établir une distinction nette entre le procédé et le produit. Ainsi, une modification de procédé dans les télécommunications visant à mettre en place un réseau intelligent pourrait permettre la commercialisation de toute une gamme de nouveaux produits tels que l'appel en attente et l'affichage des appels. L'encadré 1 présente des exemples d'innovations dans des branches du secteur des services.

\section{DIFFUSION DES INNOVATIONS TPP : NOUVEAUTÉ INSTITUTIONNELLE}

143. Il y a innovation TPP à l'échelle mondiale lorsqu'un produit ou un procédé nouveau ou amélioré est accompli pour la toute première fois. L'innovation TPP au niveau "de la firme seulement" intervient lorsqu'une firme réalise un produit ou un procédé technologiquement nouveau ou amélioré, nouveau pour elle, mais qui a déjà été réalisé dans d'autres firmes et industries.

144. Entre les deux, il y a divers degrés de diffusion des produits et des procédés technologiquement nouveaux ou améliorés. Il est possible d'en donner différentes répartitions, par exemple en fonction du marché sur lequel le produit ou le procédé est diffusé (nouveau pour le marché en question, notion que les répondants aux enquêtes peuvent aisément comprendre) ou de la région géographique (nouveau pour le pays ou pour la région, présentant un intérêt pour les pouvoirs publics) (voir chapitre 2).

\subsection{Champ couvert minimal}

145. Le présent manuel traite de tous ces niveaux dans la mesure où le niveau minimal d'entrée est "nouveau pour la firme".

146. La relation entre les deux ensembles de catégories définis jusqu'ici est présentée à la figure 3 .

147. Au cours du processus de diffusion, il se peut que le produit nouveau ou amélioré d'une firme devienne procédé nouveau ou amélioré d'une autre firme. Un modèle d'ordinateur plus puissant, par exemple, constitue un produit technologiquement amélioré pour le secteur de la bureautique, mais peut représenter un procédé technologiquement nouveau pour un cabinet d'experts-comptables. En outre, le logiciel de comptabilité utilisé avec cet ordinateur sera peut-être un produit bien connu dans le secteur des services informatiques, mais constituera un procédé entièrement nouveau pour le cabinet d'experts-comptables. 
148. La situation est plus compliquée lorsque l'on aborde les activités de services de manutention de marchandises et/ou de commerce de distribution (distribution de gros et de détail, transport et entreposage), assurant en général la diffusion de produits technologiquement nouveaux ou améliorés, qui sont conçus, produits et réalisés par leurs fournisseurs, sans que ces activités n'apportent en soi une quelconque valeur ajoutée technologique. D'une manière générale, les échanges concernant de tels produits nouveaux ou améliorés ne devraient pas être considérés comme une innovation TPP pour le grossiste, point de vente au détail ou l'entreprise de transport et d'entreposage. Cependant, si cette firme commence à traiter une gamme de produits complètement nouveaux, on peut considérer qu'il s'agit d'une innovation de produit. Par exemple, un nouveau progiciel constitue une innovation technologique de produit pour la société de services informatiques. Pour le grossiste ou le détaillant qui le distribue, c'est un nouveau produit du catalogue mais non une innovation technologique de produit, à moins que la firme n'ait jamais distribué auparavant de "produits-logiciels".

149. Il s'ensuit que, dans le secteur de la distribution, l'innovation TPP sera généralement une innovation de procédé, comme c'est le cas par exemple de l'adoption de la livraison "juste à temps" par un grossiste, ou de la gestion informatisée des stocks par un détaillant. Il est suggéré de considérer comme une innovation de procédé la diffusion d'un produit nouveau ou amélioré tel que décrit ci-dessus qui impose à l'entreprise de distribution une certaine activité technologique.

\subsection{Champ couvert au sein de la firme}

150. Les firmes peuvent exercer des activités principale, secondaire et auxiliaires, selon la définition du Système de comptabilité nationale (CEC et al., 1993).

151. Des innovations peuvent être accomplies à la fois pour les activités de production principale et secondaire d'une firme.

152. Ainsi, une société de matériel informatique peut lancer une version nettement améliorée d'un programme qu'elle commercialisera comme un produit secondaire distinct, ou encore un restaurant peut installer des machines à sous qui seront pour lui un nouveau produit secondaire de service.

153. L'innovation technologique peut concerner tant le procédé de production et/ou les produits de la firme, que les activités auxiliaires et de soutien assurées par ses services d'achat, de vente, de comptabilité, d'informatique ou d'entretien. Dans la pratique, il sera très difficile de détecter une innovation de produit dans les activités auxiliaires.

154. L'innovation technologique de procédé dans les activités auxiliaires est prise en compte.

Ainsi, l'informatisation des ventes ou du service financier d'une firme peut être considérée comme une innovation TPP. 
Figure 3. Type et degré de nouveauté et définition de l'innovation

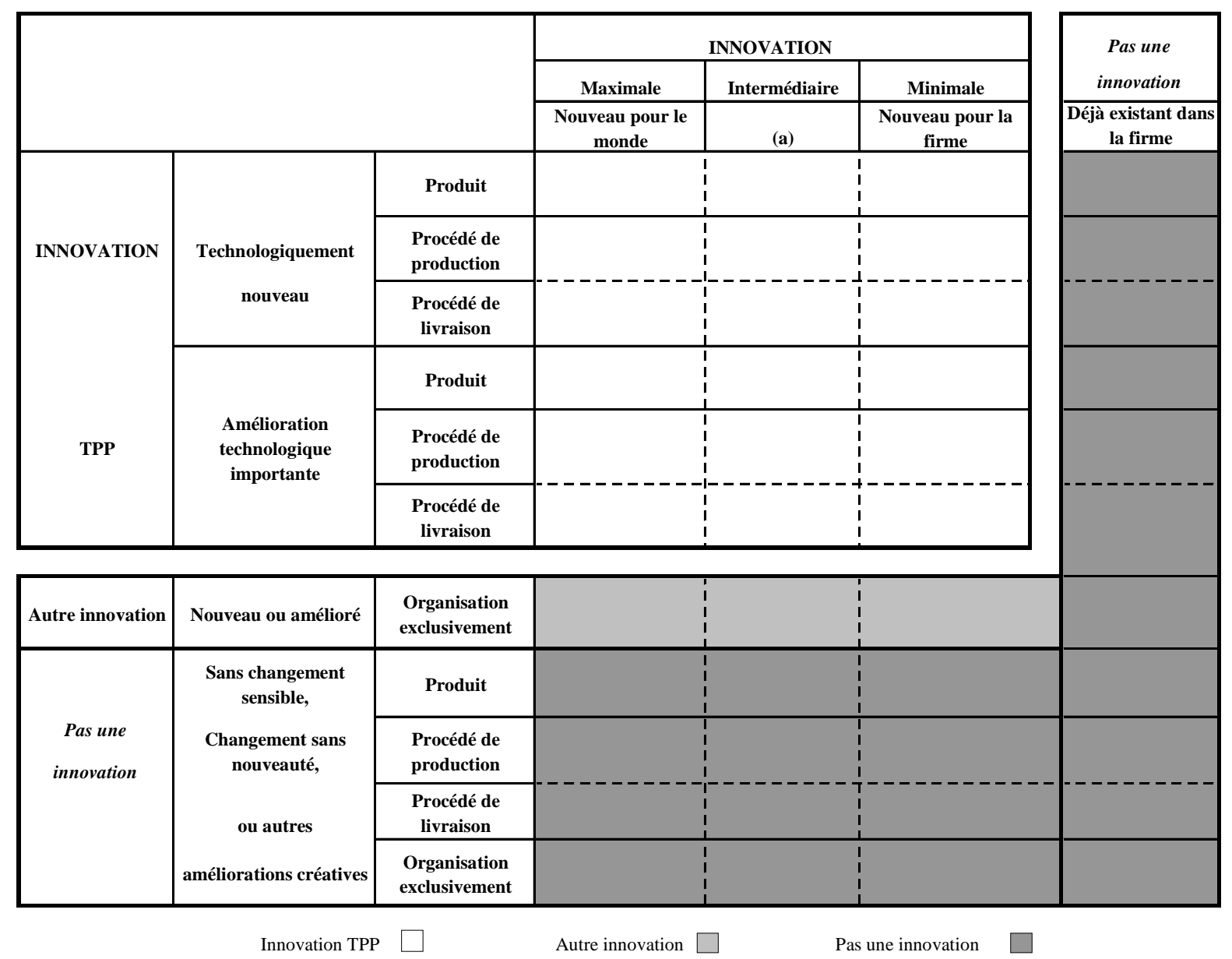

(a) Pourrait être géographique, par exemple nouveau pour le pays ou la région.

\section{DISTINCTION ENTRE L'INNOVATION TPP ET LES AUTRES CHANGEMENTS AU} SEIN DE LA FIRME OU DANS L'INDUSTRIE

155. Il importe de distinguer l'innovation TPP (voir figure 3) :

- de l'innovation dans l'organisation,

- des autres modifications apportées aux produits et aux procédés.

\subsection{Innovation dans l'organisation}

\subsubsection{Champ couvert}

156. L'innovation dans l'organisation au sein de la firme comprend :

- la mise en place de structures sensiblement modifiées dans l'organisation, 
- la mise en oeuvre de techniques avancées de gestion,

- l'adoption par la firme d'orientations stratégiques nouvelles ou sensiblement modifiées.

157. En principe, les changements dans l'organisation ne sont considérés comme des innovations que dans la mesure où ils ont un effet mesurable sur la production, par exemple une augmentation de la productivité ou des ventes. Cependant, la présente section n'a pas pour objet de préciser les frontières entre les changements dans l'organisation qui sont novateurs et ceux qui ne le sont pas. La description qui est donnée ici vise à faire la distinction entre les changements organisationnels et l'innovation TPP. On trouvera à l'annexe 2 une description plus complète qui sera utile à ceux qui souhaitent recueillir des données sur l'innovation dans l'organisation.

\subsubsection{Cas limites : changement dans l'organisation concernant les procédés dans l'industrie} manufacturière et les procédés de service

158. Alors que la réorganisation complète d'une firme constitue une "innovation dans l'organisation", la réorganisation de son installation de production peut être considérée comme une innovation TPP. Par exemple, l'introduction de systèmes de production en flux tendus doit être assimilée à une innovation de procédé dans la mesure où elle exerce un effet direct sur la production de produits destinés au marché.

159. Dans le secteur des services, l'innovation technologique de procédé comprend les moyens améliorés incorporés aux organisations et travaux courants, à la condition qu'il en résulte une variation mesurable de la production. Par exemple, l'application d'une norme de qualité telle que l'ISO 9000 ne constitue pas une innovation TPP à moins qu'elle aboutisse à une amélioration notable de la production ou de la livraison de biens ou de services.

\subsection{Autres changements concernant les produits et les procédés}

160. Il s'agit de changements qui :

- sont insignifiants, mineurs, ou n'apportent pas un degré suffisant de nouveauté ;

- apportent "d'autres améliorations créatives" lorsque la nouveauté ne concerne pas l'utilisation ou les caractéristiques objectives d'efficacité des produits, ou encore des méthodes de production et de livraison, mais plutôt leurs qualités esthétiques ou autres attributs à caractère subjectif.

161. Il existe manifestement de nombreux cas limites dans ces deux domaines, de sorte que seuls ceux qui répondent à l'enquête et/ou les personnes qui opèrent la sélection des innovations TPP à introduire dans les bases de données peuvent se prononcer de façon définitive sur la nature du changement.

\subsubsection{Exclusion de changements insignifiants ou non porteurs de nouveauté}

(a) Cessation de l'utilisation d'un procédé ou de la commercialisation d'un produit

162. Le fait qu'une firme mette fin à une activité n'est pas une innovation TPP, bien que cela puisse accroître son efficacité. Par exemple, il n'y a pas innovation TPP lorsqu'un fabricant de téléviseurs cesse de produire et de vendre un modèle avec magnétoscope intégré, ou encore lorsqu'un promoteur 
immobilier ou une entreprise de bâtiment et travaux publics cesse de construire des résidences pour retraités.

(b) Simple remplacement ou accroissement de l'équipement

163. L'achat de machines supplémentaires d'un modèle déjà installé, même si elles sont extrêmement perfectionnées, ne constitue pas une innovation technologique de procédé. Par définition, un nouveau modèle répond à des spécifications nettement améliorées, et il ne suffit pas qu'il porte un nouveau numéro ou libellé dans le catalogue du fabricant. En ce qui concerne les logiciels, par exemple, l'achat de la nouvelle version d'une série de programmes pour Windows peut être considéré comme une amélioration technologique de procédé, mais non l'acquisition de mises à jour intermédiaires qui n'améliorent pas sensiblement l'efficacité des programmes.

164. Dans ce cas, on peut faire la distinction en déterminant si le personnel concerné aura besoin d'une nouvelle formation pour pouvoir utiliser la nouvelle machine ou le nouveau logiciel. Cependant, la diffusion de nouvelles copies à l'intérieur de la firme n'entre pas dans ce cas de figure.

(c) Changements découlant uniquement de variations du prix des facteurs

165. Pour que l'on puisse parler d'innovation TPP, il faut qu'il y ait modification de la nature (ou de l'emploi) du produit ou du procédé. Une modification du prix d'un produit ou de la productivité d'un procédé qui découle exclusivement de variations du prix des facteurs de production n'est pas une innovation.

166. Ainsi, il n'y a pas innovation lorsque le prix de revient et le prix de vente d'un modèle de PC baissent uniquement parce que le prix des puces a diminué.

(d) Le cas de la production personnalisée

167. Les firmes qui ont un secteur de production personnalisée, fabriquant des articles uniques et souvent complexes à la demande du client, doivent analyser chaque produit pour déterminer s'il correspond aux définitions de l'innovation TPP énoncées plus haut. Si les attributs de ce produit unique ne diffèrent pas sensiblement de ceux des produits fabriqués antérieurement par la firme, il ne s'agit pas d'une innovation technologique de produit.

168. Dans les cas limites, on pourrait retenir comme critère d'innovation TPP le fait que la phase de planification du produit comporte la construction et l'essai d'un prototype ou d'autres activités de recherche et de développement destinées à changer au moins un attribut du produit.

(e) Modifications saisonnières et autres changements cycliques

169. Certaines branches d'activité, comme l'habillement et la chaussure, connaissent des variations saisonnières dans les types de biens ou de services fournis, variations qui peuvent s'accompagner d'un phénomène de mode pour les produits concernés (voir Section 4.2.2 ci-dessous). En général, un type de produit donné réapparaîtra sur le marché au bout d'un certain temps. On ne saurait, dans ce cas, le considérer comme une innovation, à moins qu'il n'ait été dans l'intervalle amélioré au plan technologique. Ainsi, la vente d'anoraks pour la nouvelle saison par un fabricant d'articles d'habillement n'est pas une innovation TPP à moins que par exemple, leurs doublures ne présentent des caractéristiques améliorées ; on ne peut pas considérer non plus comme une innovation TPP la réouverture annuelle du rayon ski dans un magasin. 
170. Une différenciation du produit consiste en l'adoption de modifications techniques (ou esthétiques) mineures en vue de s'introduire sur une nouvelle tranche du marché, afin d'élargir la gamme apparente de produits ou de repositionner un produit par rapport à un produit concurrent. On ne peut considérer qu'il s'agit d'une innovation de produit technologiquement amélioré que si les changements modifient sensiblement les performances ou les propriétés du produit concerné ou bien l'emploi de matériaux ou de composants qui entrent dans sa fabrication.

171. Ainsi, le changement de nom et d'emballage d'une boisson non alcoolisée existante, en vogue auprès d'un public plus âgé, pour créer un lien avec une équipe de football et toucher ainsi le marché des jeunes, n'est pas une innovation TPP.

172. Les nouveaux modèles de produits complexes, tels que des automobiles ou des téléviseurs, relèvent de la différenciation du produit, si les modifications sont d'importance mineure par rapport aux modèles précédents, en proposant par exemple une voiture équipée de radio. Si les changements sont notables, par exemple s'ils s'appuient sur des conceptions nouvelles ou des modifications techniques de sous-systèmes, les produits améliorés pourraient être considérés comme des innovations de produit technologiquement amélioré.

\subsubsection{Innovation TPP et autres améliorations créatives de produit}

173. Pour qu'il y ait innovation technologique, il faut une amélioration objective des performances d'un produit ou des modalités de livraison. Dans le cas de nombre de biens et de services vendus directement aux consommateurs ou aux ménages, la firme peut apporter des améliorations à ses produits qui les rendent plus attrayants pour les acheteurs sans modifier leurs caractéristiques "technologiques". Ces améliorations peuvent avoir une influence considérable sur les ventes de la firme, laquelle pourrait bien les considérer comme des innovations. Cependant, il ne s'agit pas d'innovations TPP.

174. Ainsi, une modification dans la production de vêtements relève en très grande partie de la mode. Pour des entreprises de ce secteur, l'adoption rapide des couleurs et de la coupe les plus récentes est essentielle pour leur compétitivité. Néanmoins, la couleur et la coupe ne changent pas les caractéristiques essentielles ou les propriétés d'un vêtement, c'est-à-dire le fait qu'il doit conserver le corps à une température appropriée, être confortable à porter et d'entretien facile. Dans ce cas, les produits technologiquement améliorés impliquent presque toujours l'emploi de nouveaux matériaux diffusés par l'industrie textile et, préalablement, par l'industrie chimique. Ainsi, l'introduction de chemises ne nécessitant pas de repassage, ou de tenues de montagne imperméables à l'eau qui évacuent la transpiration, constitue une innovation technologique de produit.

175. Dans les activités des agences et organisateurs de voyages, les services d'information et de réservation en ligne sont des innovations technologiques, mais les offres de voyages organisés sur de nouveaux thèmes ne le sont pas. L'offre d'un service téléphonique sur les trains constitue une innovation technologique, contrairement au changement de décoration du matériel roulant.

176. Pour certaines industries, le cadre dans lequel les services sont offerts est important. L'entretien ou l'amélioration de ce cadre ne relève pas de l'innovation TPP, sauf s'il est lié à une amélioration sensible et objective du service ou de la façon dont celui-ci est produit ou fourni. Par exemple, la réfection des peintures, le changement de moquettes ou une décoration tout à fait nouvelle d'un restaurant n'est pas 
une innovation TPP. En revanche, l'adoption d'un système de commande et de facturation par ordinateur, ou l'installation de fours à micro-ondes, constitue une innovation TPP.

\section{ACTIVITÉS D'INNOVATION TPP}

177. Les activités d'innovation TPP sont toutes les démarches scientifiques, technologiques, organisationnelles, financières et commerciales, y compris l'investissement dans de nouvelles connaissances, qui mènent ou visent à mener à la réalisation de produits ou de procédés technologiquement nouveaux ou améliorés. Certaines de ces activités peuvent être par elles-mêmes véritablement novatrices, tandis que d'autres, ne présentent pas de nouveauté mais sont nécessaires à la mise en oeuvre.

\subsection{Relation avec la réalisation d'innovations TPP}

178. Pendant une période donnée, les activités d'innovation TPP d'une firme peuvent être de trois types :

- réussies, en ce qu'elles aboutissent à la réalisation d'un produit ou d'un procédé technologiquement nouveau ou amélioré ;

- avortées avant la réalisation d'un produit ou d'un procédé technologiquement nouveau ou amélioré, soit parce que le projet se heurte à des difficultés, soit parce que l'idée, ou le savoir-faire, est vendue ou négocié d'une autre manière à une autre firme, ou encore parce que les conditions du marché ont changé ;

- en cours de développement, mais n'ont pas encore atteint le stade de la réalisation. Ces activités peuvent viser un produit ou un procédé nouveau ou amélioré précis, ou avoir des objectifs plus vagues, comme c'est le cas pour la recherche fondamentale ou la recherche technologique générale.

\subsection{Les composantes et la couverture des activités d'innovation TPP}

179. L'innovation est un processus complexe, comme on l'a souligné au chapitre 2, et la portée des activités nécessaires à l'accomplissement d'une innovation TPP dans une firme peut varier considérablement. Par exemple, le développement interne d'un produit électronique grand public perfectionné radicalement différent exigera des étapes plus nombreuses que l'adoption d'un procédé amélioré découlant d'une technologie incorporée à une machine pré-programmée achetée à cet effet.

180. Les activités d'innovation peuvent être menées à l'intérieur de la firme ou nécessiter l'acquisition de biens, de services ou de connaissances provenant de sources extérieures, y compris des services de conseils. Ainsi, une firme peut se procurer à l'extérieur des technologies sous forme immatérielle ou incorporée.

181. La liste ci-après des activités n'est pas exhaustive. Elle a pour but d'expliquer quand il y a lieu d'inclure certaines activités dans l'innovation TPP. On trouvera des indications plus concrètes dans le chapitre sur la mesure des dépenses consacrées à l'innovation. Les activités se rapportant à une innovation exclusivement organisationnelle sont traitées dans l'annexe 2 . 


\subsubsection{Acquisition et production de connaissances pertinentes qui sont nouvelles pour la firme}

(a) Recherche et développement expérimental

182. La recherche et le développement expérimental (R-D) englobent les travaux de création entrepris de façon systématique en vue d'accroître la somme de connaissances, y compris la connaissance de l'homme, de la culture et de la société, ainsi que l'utilisation de cette somme de connaissances pour concevoir de nouvelles applications (selon la définition donnée dans le Manuel de Frascati).

183. La construction et l'essai d'un prototype représentent souvent la phase la plus importante du développement expérimental. Un prototype est un modèle original (ou une situation d'essai) qui présente toutes les caractéristiques techniques et les performances du nouveau produit ou procédé. L'acceptation d'un prototype correspond souvent à la fin de la phase de développement expérimental et au début des phases suivantes du processus d'innovation (on trouvera des précisions supplémentaires à cet égard dans le Manuel de Frascati).

184. Le développement d'un logiciel entre dans la catégorie des activités de R-D dans la mesure où il fait intervenir un progrès scientifique ou technologique et/ou dissipe une incertitude scientifique/technologique de manière systématique.

(b) Acquisition de technologie non incorporée et de savoir-faire

185. L'acquisition à l'extérieur de technologie sous forme de brevets, inventions non brevetées, licences, divulgations de savoir-faire, marques de fabrique, études de conception, modèles et services informatiques, ainsi que d'autres services scientifiques et techniques, liés à la réalisation d'innovations TPP, à laquelle s'ajoute l'acquisition de progiciels non classés ailleurs.

(c) Acquisition de technologie incorporée

186. L'acquisition de machines et d'équipements aux performances technologiques améliorées (logiciel intégré compris) liées à des innovations technologiques de produit ou de procédé réalisés par la firme.

\subsubsection{Autres activités de préparation à la production}

(a) Outillage et ingénierie industrielle

187. Modifications des procédures de production et de contrôle de la qualité, des méthodes et des normes ainsi que des logiciels associés requis pour produire le produit technologiquement nouveau ou amélioré ou pour utiliser le procédé technologiquement nouveau ou amélioré.

(b) Etudes de conception industrielle non classées ailleurs

188. Les plans et dessins destinés à définir les procédures, les spécifications techniques et les caractéristiques de fonctionnement nécessaires à la production de produits technologiquement nouveaux et à la mise en oeuvre de procédés nouveaux. 
189. L'acquisition de bâtiments, ou de machines, outillage et équipements - ne comportant pas d'amélioration de performances technologiques - qui sont nécessaires à la réalisation de produits ou de procédés technologiquement nouveaux ou améliorés, par exemple, une machine à mouler ou une machine d'emballage supplémentaire pour produire et livrer un lecteur de CD-ROM technologiquement amélioré.

(d) Démarrage de la production

190. Il peut s'agir de modifications apportées aux produits ou aux procédés, du recyclage du personnel pour le former aux nouvelles techniques ou à l'utilisation de nouvelles machines, et de tout essai de production ne faisant pas déjà partie des activités de R-D.

\subsubsection{Commercialisation de produits nouveaux ou améliorés}

191. Les activités associées au lancement d'un produit technologiquement nouveau ou amélioré. Elles peuvent inclure des études de marché préliminaires, les essais de commercialisation et la publicité accompagnant le lancement, mais ne couvrent pas la mise en place de réseaux de distribution pour la commercialisation des innovations.

\subsection{Cas limites}

192. De tous les types de travaux susmentionnés, seules la R-D et l'acquisition de machines dans lesquelles est incorporée une technologie nouvelle sont considérées, par définition, comme des activités d'innovation TPP. Les autres peuvent en faire partie ou non, selon leur finalité.

\subsubsection{La conception}

193. La conception industrielle est un élément essentiel du processus d'innovation TPP. Bien qu'elle soit placée ci-dessus dans la même sous-section que l'outillage, l'ingénierie industrielle et le démarrage de la production, elle peut aussi faire partie de l'élaboration initiale du produit ou du procédé, autrement dit comprise dans la recherche et le développement expérimental, ou encore s'avérer nécessaire pour la commercialisation de produits technologiquement nouveaux ou améliorés.

194. Les activités de conception artistique relèvent des activités d'innovation TPP si elles sont entreprises dans le cadre de la réalisation d'un produit ou d'un procédé technologiquement nouveau ou amélioré. Elles ne le sont pas si elles sont entreprises pour une autre amélioration créative du produit, par exemple simplement pour en améliorer l'aspect, sans objectivement modifier en rien sa performance.

\subsubsection{La formation}

195. La formation est une activité d'innovation TPP lorsqu'elle est nécessaire à la réalisation d'un produit ou d'un procédé technologiquement nouveau ou amélioré, par exemple pour que les travailleurs d'une crémerie soient capables d'identifier la consistance voulue d'un nouveau type de yaourts, qu'un directeur commercial puisse comprendre les caractéristiques d'un système de freinage amélioré sur un nouveau modèle de voiture afin de préparer son lancement sur le marché, ou encore pour que le 
personnel sache utiliser différents programmes sous Windows après la mise en place dans la firme d'un réseau d'ordinateurs personnels dans un environnement Windows.

196. La formation dans une firme n'est pas une activité d'innovation TPP si elle n'est liée qu'à des activités de la catégorie "innovation organisationnelle" ou "autres améliorations créatives de produit", ou si elle n'est pas axée sur une amélioration précise de la productivité au niveau de la firme. Voici des exemples d'activités qui ne relèvent pas de l'innovation TPP : la formation aux méthodes de production existantes destinée aux nouveaux employés, le perfectionnement en général des individus (pour les cadres, par exemple), la formation permanente en informatique, les cours de langue.

\subsubsection{La commercialisation}

197. La commercialisation est une activité d'innovation TPP quand elle est nécessaire à la réalisation d'un produit technologiquement nouveau ou amélioré (ou, plus rarement, d'un nouveau procédé). Ce n'est pas une activité d'innovation TPP quand elle est entreprise à des fins d'innovation purement organisationnelle, par exemple une campagne visant à promouvoir la nouvelle structure ou l'image de marque d'une firme, ou dans le cadre d'une autre amélioration créative de produit, par exemple la publicité pour la collection printanière dans le domaine du vêtement, ou pour maintenir la part du marché de produits qui, pour l'essentiel, restent inchangés, savons en poudre, par exemple.

\subsubsection{Les logiciels}

198. Le développement, l'acquisition, l'adaptation et l'utilisation des logiciels pénètrent l'ensemble des activités d'innovation TPP. D'une part, le développement de logiciels nouveaux ou sensiblement améliorés, en tant que produit commercial ou à usage interne (innovation TPP en soi), nécessite des activités de recherche et de développement expérimental ainsi que tout un éventail d'activités d'innovation post-R-D. D'autre part, bon nombre des activités d'innovation en vue d'autres innovations TPP supposent l'utilisation de logiciels, et donc leur acquisition et leur adaptation.

\section{LA FIRME INNOVANTE TPP}

199. La firme innovante TPP est la firme qui a réalisé un ou plusieurs produits, procédés ou combinaisons de produits et de procédés technologiquement nouveaux ou sensiblement améliorés pendant la période sous revue. C'est une firme qui a mené des activités d'innovation TPP réussies (voir section 5.1 ci-dessus) pendant cette période.

200. Une firme qui a mené des activités d'innovation TPP avortées n'est pas classée dans cette catégorie, ni une firme qui a, à la fin de la période considérée, des travaux d'innovation TPP en cours mais qui n'ont pas encore débouché sur une réalisation.

201. En théorie, toutes les firmes qui se sont créées pendant la période considérée ont accompli de nouveaux produits ou procédés. En pratique, il est recommandé ce qui suit : 
Par convention, les firmes innovantes comprennent :

-- les firmes qui existaient déjà au début de la période considérée et qui ont réalisé un ou plusieurs produits ou procédés technologiquement nouveaux (ou améliorés) pour ladite firme pendant cette période ;

-- les firmes qui ont été créées pendant la période considérée et qui

--lors de leur création, ont réalisé un ou plusieurs produits ou procédés qui sont technologiquement nouveaux (ou améliorés) pour le marché sur lequel opère la firme ;

--après leur création, ont réalisé un ou plusieurs produits ou procédés au cours de la période considérée, qui sont technologiquement nouveaux (ou améliorés) pour ladite firme.

Il est admis que l'application de ce classement peut se révéler difficile dans la pratique. On trouvera au chapitre 5, section 3 de plus amples renseignements, ainsi que des recommandations sur le choix des périodes appropriées.

\section{THÉORIE ET PRATIQUE}

202. Les définitions énoncées ci-dessus décrivent en détail les types de données qui doivent être collectées ou rassemblées afin de s'assurer qu'elles sont précises et, dans toute la mesure du possible, logiquement cohérentes. Il appartient à ceux qui conçoivent les formulaires d'enquête de s'appuyer sur ces définitions pour exprimer les concepts de façon appropriée et significative pour les répondants aux enquêtes dans les industries concernées, par exemple lorsqu'est expliqué le concept de performances sensiblement améliorées des produits en des termes appropriés pour les firmes appartenant à certains secteurs de services où le mot "technologique" en soi risque de prêter à confusion. 


\section{Chapitre 4}

\section{CLASSIFICATIONS INSTITUTIONNELLES}

\section{DÉMARCHE ADOPTÉE}

203. La démarche institutionnelle est essentiellement axée sur les caractéristiques de la firme innovante, et l'ensemble des caractéristiques des activités d'innovation, ainsi que leurs inputs et leurs outputs, sont affectés à une catégorie ou à une sous-catégorie selon l'activité principale de cette unité.

\section{LES UNITÉS}

204. Il convient d'établir une distinction claire entre unités déclarantes et unités statistiques. L'unité déclarante est l'entité auprès de laquelle les éléments d'information recherchés sont recueillis. Celle-ci peut varier d'un secteur à l'autre et d'un pays à l'autre selon les structures institutionnelles, le statut juridique de la collecte de données, les traditions, les priorités nationales et les moyens d'enquête. Il est donc presque impossible de formuler des recommandations internationales sur l'unité déclarante pour les enquêtes sur l'innovation. Toutefois, lorsque les pays fournissent des données statistiques destinées à des comparaisons internationales, l'unité déclarante devrait être spécifiée.

205. L'unité statistique est l'entité pour laquelle les données demandées sont compilées. Il peut s'agir d'une unité d'observation pour laquelle des informations sont reçues ou des statistiques sont compilées, ou d'une unité analytique que les statisticiens créent en décomposant ou en regroupant des unités d'observation au moyen d'estimations ou d'imputations, afin de fournir des données plus détaillées et/ou plus homogènes qu'ils ne pourraient le faire autrement.

206. Dans la mesure du possible, l'unité statistique devrait être uniforme dans tous les pays ; mais, dans la pratique, cette uniformité n'est jamais réalisée. Ceci s'explique généralement par les différences de structure entre pays et peut aussi s'expliquer par l'interaction avec l'unité déclarante. Si l'unité déclarante est plus grande que l'unité statistique, il peut être difficile de répartir les données entre les unités de classification appropriées.

207. Etant donné la manière dont les activités d'innovation sont généralement organisées, l'unité du type "entreprise" constitue, dans de nombreux cas, l'unité statistique la mieux appropriée pour les enquêtes sur l'innovation. L'entreprise ${ }^{27}$ ou l'entité juridique définie aux paragraphes 78 et 79 de la CITI Rév. 3 est l'unité appropriée. Cependant, lorsqu'il s'agit de grandes entreprises, qui mènent des activités dans plusieurs secteurs industriels, une unité plus petite, telle que l'unité fonctionnelle " "une entreprise ou une partie d'une entreprise qui exerce un seul type d'activité économique sans être limitée à la région géographique où l'activité est exercée", autrement dit l'unité d'activité économique (UAE) ${ }^{29}$, serait mieux appropriée. 
208. Pour l'analyse régionale, l'unité locale ${ }^{30}$ ou une unité équivalente peut se révéler plus indiquée ${ }^{31}$. Il convient de garder présent à l'esprit que les informations relatives à certaines variables ne devraient pas être collectées au niveau des unités locales (ou analogues) car elles se rapportent directement à l'entreprise. Les informations sur les objectifs des innovations en sont un exemple. Elles concernent les décisions stratégiques au niveau de l'entreprise et ne peuvent pas être rattachées à des unités locales.

209. Dans les enquêtes sur l'innovation, les entreprises multinationales, pour lesquelles les différentes parties du processus d'innovation sont localisées dans des pays différents, mériteront peut-être un traitement spécial. Lorsque l'on prend les unités nationales comme unités statistiques, il n'est pas tenu compte des liens existant entre des unités d'entreprises multinationales qui se trouvent dans différents pays. Par conséquent, les résultats nationaux risquent d'être trompeurs. Des solutions à ce problème devraient être élaborées dans le cadre des débats plus généraux actuellement consacrés à la mondialisation.

\section{CLASSIFICATION SELON L'ACTIVITÉ ÉCONOMIQUE PRINCIPALE}

210. On peut ventiler les unités statistiques des enquêtes sur l'innovation en fonction de variables fort différentes. La variable la plus importante peut-être est l'activité économique principale de l'unité statistique (1'"industrie"). La Classification internationale type, par industrie, de toutes les branches d'activité économique (CITI, Rév. 3 ) et la Nomenclature industrielle qui doit être utilisée dans les Communautés Européennes (NACE, Rév. 1) constituent respectivement des classifications internationales appropriées à cet effet.

211. Les critères de classification de ces unités statistiques par activité principale devraient être déterminés par "la catégorie de la CITI (NACE) qui correspondra à son activité principale, ou au groupe de ses activités principales" ${ }^{32}$. Selon la CITI, il faudrait, pour déterminer l'activité principale, se référer à la valeur ajoutée des produits vendus ou des services fournis du fait des activités considérées. Si cela n'est pas possible, l'activité principale pourra être déterminée soit en fonction de la production brute des produits vendus ou des services fournis par chaque activité, soit par le nombre de personnes employées à ces activités ${ }^{33}$.

212. La liste de classification proposée figure au tableau 1, qui contient une présentation spéciale des divisions, groupes et classes de la CITI, Rév. 3 / NACE, Rév. 1 en vue de l'établissement de statistiques sur l'innovation. Ce tableau doit être considéré comme donnant la présentation de base qui pourrait faire l'objet d'une décomposition ou d'une agrégation plus poussée à des fins spécifiques.

\section{CLASSIFICATION SELON LA TAILLE}

213. L'autre classification essentielle prend comme critère la taille des unités statistiques des enquêtes sur l'innovation. Bien que différentes variables puissent être utilisées pour définir la taille d'une telle unité statistique dans les enquêtes sur l'innovation, il est recommandé de mesurer cette taille sur la base des effectifs de personnel. Cette recommandation est conforme aux propositions analogues figurant dans les autres manuels de type Frascati. Compte tenu des strates nécessaires pour les enquêtes par échantillons (voir chapitre 7), et également du fait que des activités d'innovation sont menées dans des unités de toutes tailles mais, contrairement à la R-D, aussi très répandues dans des unités de petite et de moyenne importance, les catégories suivantes de tailles sont recommandées : 
Tableau 1. Classification par industrie proposée pour les enquêtes sur l'innovation dans le secteur des entreprises fondée sur la CITI Rév.3 et la NACE Rév.1

\begin{tabular}{|c|c|c|}
\hline Intitulé & $\begin{array}{c}\text { CITI Rév.3 } \\
\text { Division/ Groupe/Classe }\end{array}$ & $\begin{array}{c}\text { NACE Rév.1 } \\
\text { Div./Groupe/Classe }\end{array}$ \\
\hline ACTIVITÉS DE FABRICATION & 15 à 37 & 15 à 37 \\
\hline Produits alimentaires et boissons & 15 & 15 \\
\hline Produits à base de tabac & 16 & 16 \\
\hline Textiles & 17 & 17 \\
\hline Articles d'habillement et fourrures & 18 & 18 \\
\hline Articles en cuir et chaussures & 19 & 19 \\
\hline Bois et liège (sauf meubles) & 20 & 20 \\
\hline Carton, papier et articles & 21 & 21 \\
\hline Edition, impression et reproduction d'enregistrements & 22 & 22 \\
\hline Coke, raffinerie pétrole et combustibles nucléaires & 23 & 23 \\
\hline Produits chimiques & 24 & 24 \\
\hline Produits chimiques moins pharmaceutiques & 24 moins 2423 & 24 moins 24.4 \\
\hline Produits pharmaceutiques & 2423 & 24.4 \\
\hline Caoutchouc et matières plastiques & 25 & 25 \\
\hline Produits minéraux non métalliques & 26 & 26 \\
\hline Produits métallurgiques de base & 27 & 27 \\
\hline Produits métallurgiques de base ferreux & $271+2731$ & 27.1 à $27.3+27.51 / 52$ \\
\hline Produits métallurgiques de base non ferreux & $272+2732$ & $27.4+27.53 / 54$ \\
\hline Ouvrages en métaux (sauf machines et matériels) & 28 & 28 \\
\hline Machines, nca & 29 & 29 \\
\hline Machines de bureau, comptabilité et traitement inform. & 30 & 30 \\
\hline Machines électriques & 31 & 31 \\
\hline Equipements électroniques (Radio, TV et communications) & 32 & 32 \\
\hline Composants électroniques (semi-conducteurs inclus) & 321 & 32.1 \\
\hline Appareils radio, $T V$ et de communication & 32 moins 321 & 32 moins 32.1 \\
\hline Instruments médicaux, de précision et d'optique et horlogerie & 33 & 33 \\
\hline Véhicules automobiles & 34 & 34 \\
\hline Autres matériels de transport & 35 & 35 \\
\hline Navires & 351 & 35.1 \\
\hline Aérospatiale & 353 & 35.3 \\
\hline Autres équipements de transport & $352+359$ & $35.2+35.4+35.5$ \\
\hline Meubles, autres fabrications nca & 36 & 36 \\
\hline Meubles & 361 & 36.1 \\
\hline Autres activités de fabrication nca & 369 & 36.2 à 36.6 \\
\hline Récupération & 37 & $37-1$ \\
\hline DISTRIBUTION D'ÉLECTRICITE, GAZ ET EAU & $40+41$ & $40+41$ \\
\hline CONSTRUCTION & 45 & 45 \\
\hline SERVICES COMMERCIALISÉS & 50 à 99 & 50 à 99 \\
\hline Commerce (gros et détail) de véhicules automobiles et réparation & 50 & 50 \\
\hline Commerce de gros (sauf véhicules automobiles) & 51 & 51 \\
\hline Commerce de détail (sauf véhicules automobiles) et réparation & 52 & 52 \\
\hline Hôtels et restaurants & 55 & 55 \\
\hline Transports terrestres et par conduites & 60 & 60 \\
\hline Transports par eau & 61 & 61 \\
\hline Transports aériens & 62 & 62 \\
\hline Activités annexes de transports, entreposage, agences de voyage & 63 & 63 \\
\hline Postes \& télécommunications & 64 & 64 \\
\hline Postes & 641 & 64.1 \\
\hline Télécommunications & 642 & 64.2 \\
\hline Intermédiation financière (assurances incluses) & 65 à 67 & 65 à 67 \\
\hline Immobilier, locations & 70 à 71 & 70 à 71 \\
\hline Informatique et activités connexes & 72 & 72 \\
\hline Production de logiciels & 722 & 72.2 \\
\hline Autres activités informatiques & 72 moins 722 & 72 moins 72.2 \\
\hline Recherche-développement & 73 & 73 \\
\hline Autres activités de services aux entreprises & 74 & 74 \\
\hline Architecture, ingénierie et autres activités techniques & 74.2 & 742 \\
\hline
\end{tabular}




\section{Classification des unités statistiques pour les enquêtes sur l'innovation selon la taille}

\section{Effectifs de personnel :}

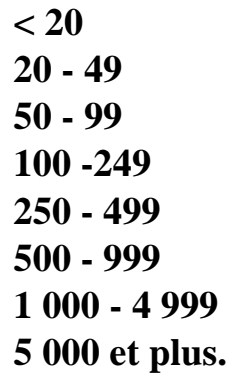

\section{AUTRES CLASSIFICATIONS}

\subsection{Type d'institution}

214. Une autre classification utile des unités statistiques utilisées pour les enquêtes sur l'innovation pourrait être par type d'institution. Cette ventilation semble particulièrement importante quand l'unité statistique est du type entreprise, et étant donnée l'internationalisation croissante des activités d'innovation. Compte tenu de ces considérations et d'une proposition semblable pour les statistiques de la R-D dans le Manuel de Frascati, il est recommandé que, lorsqu'elles sont les unités statistiques des enquêtes sur l'innovation, les entreprises soient classées de la façon suivante:

Classification des unités statistiques pour les enquêtes sur l'innovation selon les types d'institution :

- entreprise privée :

- entreprise nationale ;

- entreprise multinationale ;

- entreprise publique ${ }^{34}$;

- autres unités.

Pour définir les entreprises privées multinationales, on peut suivre la démarche adoptée dans le Manuel de Frascati, où les entreprises multinationales sont définies comme comportant une "participation étrangère" de plus de 50 pour cent ${ }^{35}$.

\subsection{Autres classifications}

215. De nombreuses autres variables ont été proposées à des fins d'analyse pour servir de base à la ventilation des unités statistiques des enquêtes sur l'innovation. Citons notamment :

- la forme d'activité, avec des catégories telles que : 
- pour le industries manufacturières : production en série / production personnalisée / industrie de transformation ;

- pour les services : à forte intensité capitalistique (transports aériens et maritimes, par exemple) / gros utilisateurs de moyens informatiques (services d'intermédiation financière ou d'études de conception, par exemple) / professionnels (activités de conseil ou activités juridiques, par exemple) / à forte proportion de main-d'oeuvre qualifiée (services de restaurants, coiffure, etc.: en général, services personnels) / à forte intensité de main-d'oeuvre ;

- le type de biens produits, avec des catégories : biens de consommation / biens intermédiaires / biens d'équipement ;

- l'intensité en activités de $\boldsymbol{R}-\boldsymbol{D}$, soit le rapport entre les dépenses de R-D et les ventes (qu'il est possible de calculer à partir des informations recueillies dans l'enquête sur l'innovation);

- la part des exportations, soit les exportations de l'entreprise exprimées par rapport aux ventes (les données correspondantes sont également recueillies dans le cadre de l'enquête sur l'innovation); ou

\section{- l'appartenance à un groupe.}

216. Ces ventilations pourraient être utiles à des objectifs spécifiques, mais ne sont pas généralement recommandées, car elles se réfèrent habituellement à des sous-populations. Un exemple permettra peutêtre de clarifier la question. La classification par intensité en activités de R-D n'est recommandée que si l'analyse porte sur les unités innovantes exécutant des travaux de R-D. Des unités de certaines branches d'activité, de même que des unités de petite et moyenne importance, sont particulièrement innovantes, bien qu'elles n'exercent pas d'activités de R-D. Si on applique une classification par intensité en activités de R-D à toutes les unités statistiques des enquêtes sur l'innovation, on obtient un grand groupe d'unités pour lesquelles l'intensité en activités de R-D est nulle, alors que leurs activités d'innovation sont tout à fait hétérogènes. 


\section{Chapitre 5}

\section{MESURE DE CERTAINS ASPECTS DU PROCESSUS D'INNOVATION}

217. Un certain nombre d'aspects du processus d'innovation seront examinés dans ce chapitre. En raison de l'expérience acquise à l'occasion d'enquêtes, il est désormais possible de recommander un ensemble de questions mises à l'essai et testées, qui s'avèrent utiles à des fins analytiques. Bien entendu, il faut circonscrire cette liste dans certaines limites, sans cela la charge que représentent les réponses pour les entreprises serait trop contraignante. Simultanément, on a aussi suggéré quelques autres questions facultatives pour être testées dans des enquêtes nationales.

218. Le processus d'innovation trouve son point de départ dans les objectifs de la firme, et est facilité ou entravé par divers facteurs. Les types d'innovations qui en résultent peuvent être décrits de différentes manières. Les indicateurs les plus importants (mais aussi les plus ardus et controversés) sont peut-être ceux qui décrivent l'influence de l'innovation sur les performances de la firme. Des indicateurs supplémentaires décrivent la diffusion de l'innovation et d'autres thèmes connexes tels que la $\mathrm{R}-\mathrm{D}$, le dépôt de brevets et l'acquisition/diffusion de technologie.

219. Les indicateurs peuvent se présenter sous forme de données binaires OUI/NON : le facteur est important/pas important. Ou bien, ils permettent de classer les facteurs par ordre d'importance sur une échelle ordinale : en commençant par déterminer si un facteur est pertinent ou non $(0=$ pas pertinent $)$, puis en allant de 1 (pas important) à 5 (très important), ou encore de 1 (pas important) à 3 (important).

220. Un certain nombre d'indicateurs sont décrits dans ce qui suit. Il peut en réalité s'avérer impossible ou irréalisable de les inclure tous dans une seule enquête. Un grand nombre de question aurait probablement pour effet de faire baisser le taux de réponse et le coût pourrait bien être trop élevé. En conséquence, lorsque les résultats des enquêtes nationales doivent servir à des comparaisons internationales, il importe de sélectionner un ensemble de questions les plus utiles et couramment utilisées, qui soient fondées sur des définitions communes.

\section{OBJECTIFS DE L'INNOVATION}

221. Il est recommandé de déterminer les raisons qui amènent une firme à se lancer dans une activité d'innovation en se fondant sur ses objectifs économiques du point de vue des produits et des marchés, et sur la manière dont elle évalue un certain nombre de buts que le processus d'innovation peut rendre accessibles. La question devrait se rapporter à toutes ses activités d'innovation. En général, plusieurs objectifs seront pertinents.

\section{Objectifs économiques de l'innovation}

- remplacer les produits qui sont supprimés ;

- étendre la gamme de produits : 
- dans le principal domaine de produits;

- hors du principal domaine de produits ;

- mettre au point des produits sans danger pour l'environnement ;

- maintenir la part du marché ;

- accroître la part du marché ;

- ouvrir de nouveaux marchés :

- à l'étranger ;

- dans de nouveaux groupes cibles nationaux;

- conférer davantage de souplesse à la production ;

- abaisser les coûts de production en :

- réduisant les coûts salariaux par unité produite ;

- diminuant la consommation de matériaux ;

- diminuant la consommation d'énergie ;

- réduisant le taux de rejet ;

- réduisant les coûts de conception des produits ;

- réduisant les délais de production ;

- améliorer la qualité d'un produit ;

- améliorer les conditions de travail ;

- réduire les atteintes à l'environnement. 


\section{FACTEURS FACILITANT OU FREINANT L'INNOVATION}

222. Deux ensembles de facteurs seront en l'occurrence pris en considération :

- le processus d'innovation est facilité par des sources d'information diverses : des sources internes (au sein de la firme), des sources externes provenant du marché, des institutions d'enseignement ou de recherche, et des informations généralement accessibles ;

- l'innovation peut être freinée par des facteurs économiques, des facteurs liés à la firme, ainsi que par une variété d'autres encore.

\section{Il est recommandé de collecter des informations sur ces deux aspects.}

223. Ces ensembles de facteurs se chevauchent dans une certaine mesure, de sorte qu'un facteur peut faciliter le processus dans un cas et être un obstacle dans l'autre. La question doit se rapporter à l'ensemble des activités d'innovation de la firme.

\subsection{Sources d'information pour l'innovation}

224. La liste suivante recense les sources que l'on a jugé pertinentes dans un certain nombre d'enquêtes. Elle peut être modifiée pour répondre aux besoins nationaux.

\section{Sources d'information}

- Sources internes au sein de la firme ou du groupe :

- activités internes de R-D ;

- commercialisation ;

- production ;

- autres sources internes.

\section{- Sources externes/sources commerciales :}

- concurrents ;

- acquisition de technologie incorporée ;

- acquisition de technologie non incorporée ;

- clients ;

- sociétés de conseil ;

- fournisseurs d'équipements, de matériaux, de composants et de logiciels. 


\section{- Institutions d'enseignement/de recherche :}

- institutions d'enseignement supérieur ;

- instituts de recherche publics ;

- instituts de recherche privés.

- Informations généralement accessibles :

- publications de brevets ;

- conférences, réunions et revues professionnelles ;

- foires et expositions.

225. Certaines de ces rubriques peuvent, si on le souhaite, être subdivisées en sources nationales et sources étrangères.

\subsection{Facteurs freinant les activités d'innovation}

226. La liste ci-après énumère les obstacles ou barrières à l'innovation qui ont été considérés comme pertinents dans un certain nombre d'enquêtes. Il peut s'agir de raisons pour ne pas démarrer du tout une activité d'innovation, ou de raisons qui n'ont pas permis aux activités d'innovation d'aboutir aux résultats escomptés. La liste peut être modifiée en fonction des besoins nationaux.

\section{Facteurs freinant les activités d'innovation}

\section{- Facteurs économiques :}

- risques perçus comme excessifs ;

- coûts trop élevés ;

- absence de sources appropriées de financement;

- durée excessive du temps de retour de l'investissement dans l'innovation.

\section{- Facteurs propres à la firme :}

- potentiel d'innovation (R-D, études de conception, etc.) insuffisant ;

- manque de personnel qualifié ;

- manque d'information sur la technologie ;

- manque d'information sur les marchés ; 
- dépenses d'innovation difficiles à maîtriser ;

- résistance aux changements dans la firme ;

- disponibilité de services externes insuffisantes ;

- absence de possibilités de coopération.

\section{- Autres raisons :}

- absence de possibilités technologiques;

- absence d'infrastructure ;

- pas besoin d'innover en raison d'innovations antérieures ;

- faiblesse de la protection des droits de propriété ;

- législation, normes, réglementation, standards, fiscalité ;

- manque d'intérêt des clients pour des produits et des procédés nouveaux.

\section{IDENTIFICATION DES FIRMES INNOVANTES TPP}

227. Du point de vue de la politique, les indicateurs des conséquences du processus d'innovation constituent peut-être les résultats les plus intéressants des enquêtes sur l'innovation. Ils sont aussi ceux qui posent le plus de problèmes.

228. L'indicateur le plus simple se rapporte à la population des firmes innovantes, telle qu'elle est définie au chapitre 3, section 6. Il s'obtient en dénombrant les firmes qui ont accompli des activités d'innovation TPP réussies au cours des trois dernières années; en font partie les firmes qui existaient au début de la période considérée et qui ont accompli des innovations TPP nouvelles (ou améliorées) pour la firme en question pendant cette période, ainsi que les firmes qui ont démarré leurs activités au cours de la période considérée et qui, au moment de leur création, ont accompli des innovations TPP qui sont nouvelles (ou améliorées) pour le marché sur lequel la firme en question opère, ou encore qui, après leur création, ont accompli des innovations TPP qui sont nouvelles (ou améliorées) pour la firme pendant la période considérée.

229. Les firmes ayant mené des activités d'innovation TPP avortées ne sont pas prises en compte, comme ne le sont pas non plus celles qui, à la fin de la période considérée, mènent encore des travaux d'innovation TPP qui n'ont pas encore abouti à une réalisation.

230. En même temps, il est recommandé de dénombrer séparément les firmes qui ont mené des activités d'innovation pendant la période de référence, mais qui n'ont pas introduit d'innovation, que ce soit à cause des projets avortés ou de la durée des projets, afin de préserver la relation avec les dépenses consacrées à l'ensemble des activités d'innovation et d'obtenir le total des dépenses engagées. Les caractéristiques de ce groupe peuvent se révéler très différentes de celles des firmes qui n'ont pas entrepris d'activité d'innovation. 
231. Il conviendrait de poser une question éliminatoire sur les résultats des activités d'innovation afin de différencier les unités innovantes des unités non innovantes, et il faudrait aussi recueillir des informations sur les mutations structurelles intervenues au sein de la firme pendant la période de référence (notamment, la date à laquelle ces changements se sont produits).

\section{IMPACT DES INNOVATIONS SUR LES PERFORMANCES DES FIRMES}

232. Divers indicateurs peuvent être utilisés pour mesurer l'incidence que les innovations exercent sur l'efficacité de la firme. Ces indicateurs sont :

- la proportion des ventes imputable aux produits technologiquement nouveaux ou améliorés ;

- les résultats des activités d'innovation ;

- l'incidence de l'innovation sur l'utilisation des facteurs de production.

\subsection{Proportion des ventes imputable aux produits technologiquement nouveaux ou améliorés}

233. Les enquêtes sur l'innovation exécutées à ce jour comportaient pour la plupart une question relative à la part des ventes et des exportations imputable à des produits technologiquement novateurs mis sur le marché au cours des trois dernières années. Les résultats obtenus au moyen de cette question ont été encourageant malgré certains problèmes d'interprétation.

234. Lors de l'établissement de cet indicateur, les firmes créées pendant la période de référence doivent être traitées séparément, car les produits nouveaux représenteront, par définition, la totalité de leurs ventes. Pour ces firmes, seuls les produits nouveaux pour le marché sur lequel la firme opère (voir ci-dessous) sont pris en compte. Les firmes qui ont démarré leurs activités par suite de fusions, de scissions et d'autres sortes de réorganisations ne devraient pas être traitées comme des firmes récemment créées si elles menaient des activités similaires auparavant.

\section{Il est recommandé de poser cette question comme suit :}

\section{Part en pourcentage des ventes imputables à :}

- des produits technologiquement nouveaux (tels qu'ils sont définis au chapitre 3, section 2.1) commercialisés au cours des trois dernières années ;

- des produits technologiquement améliorés (tels qu'ils sont définis au chapitre 3, section 2.1) commercialisés au cours des trois dernières années ;

- des produits technologiquement inchangés, ou n'ayant fait l'objet que d'une différenciation de produit, mais dont les méthodes de production ont changé au cours des trois dernières années (voir chapitre 3, section 2.2) ;

- des produits technologiquement inchangés, ou n'ayant fait l'objet que d'une différenciation de produit et dont les méthodes de production sont demeurées inchangées au cours des trois dernières années. 
236. Les ventes imputables aux produits technologiquement nouveaux et aux produits technologiquement améliorés pourraient être, en outre, ventilées selon qu'il s'agit :

- de ventes dues à des produits qui sont nouveaux ou technologiquement améliorés pour le marché sur lequel la firme opère ;

- des ventes dues à des produits nouveaux ou technologiquement améliorés pour la firme seulement.

237. Les répondants devraient, de préférence, fournir les estimations des pourcentages qui leur paraissent les plus proches de la réalité. Lors de la présentation des résultats par industrie, taille des firmes, etc., ces pourcentages devraient être pondérés par les ventes.

238. Ces indicateurs sont directement influencés par la durée de vie des produits. Ils sont probablement supérieurs dans les groupes de produits dont les cycles de vie sont brefs et pour lesquels on peut s'attendre que l'innovation intervienne plus souvent. En l'occurrence, toutefois, cette sorte d'innovation n'est pas nécessairement la plus importante, ni technologiquement la plus avancée. Une grande proportion de ventes de produits technologiquement nouveaux ou notablement modifiés n'est donc pas nécessairement révélatrice d'un taux d'innovation élevé.

239. Afin de tenir compte des effets de la durée de vie du produit sur cet indicateur, il est suggéré de demander à l'entreprise de fournir une estimation de la longueur moyenne des cycles de vie de ses produits. Ces informations pourraient être utilisées pour pondérer les parts en pourcentage suggérées plus haut. Un autre moyen de poser cette question consiste à demander à quelle fréquence la firme adopte généralement des innovations.

240. D'autres facteurs doivent également être envisagés lors de l'interprétation des données obtenues au moyen de ces indicateurs :

- les firmes faisant de la production personnalisée auront souvent une plus forte proportion de produits technologiquement nouveaux ou notablement améliorés que les firmes qui font de la production par lots ou en séries, ou qui relèvent des industries de transformation ;

- les firmes plus jeunes auront une plus forte proportion de produits technologiquement nouveaux que des firmes plus anciennes ;

- les firmes ayant pour objectif de remplacer des produits qui sont supprimés (voir section 1 de ce chapitre) enregistreront des plus fortes proportions de produits technologiquement nouveaux que des firmes dont le but est d'étendre leur gamme de produits.

\subsection{Résultats de l'effort d'innovation}

241. Afin de brosser un panorama des effets de l'innovation sur l'efficacité générale, il est suggéré de recueillir certaines données générales sur l'entreprise, concernant le début et la fin de la période de trois ans considérée :

- les ventes des années t et $\mathrm{t}-2$;

- les exportations des années t et $\mathrm{t}-2$; 
- les effectifs de personnel des années t et t-2;

- la marge d'exploitation des années t et t-2.

242. Ces données pourraient être recueillies par le biais de l'enquête sur l'innovation, ou tirées d'autres sources disponibles. En comparant ces indicateurs relatifs à la population des firmes innovantes et non innovantes à ceux des entreprises, on pourrait obtenir des informations qui revêtent un intérêt considérable.

243. Les enquêtes sur panel offrent d'intéressantes possibilités d'associer des variables d'innovation à d'autres variables concernant la firme, afin d'analyser les résultats de l'innovation.

\subsection{Impact de l'innovation TPP sur l'utilisation des facteurs de production}

244. L'un des résultats de l'innovation, en particulier de l'innovation de procédé, consiste habituellement en un changement dans la fonction de production, c'est-à-dire dans l'utilisation des facteurs de production.

245. Il est suggéré d'inclure une question sur la manière dont les innovations TPP ont influé sur l'utilisation des facteurs de production, à savoir l'utilisation de main-d'oeuvre, la consommation de matériaux, la consommation d'énergie et l'utilisation de capital fixe.

246. On pourrait obtenir cette information plus simplement en demandant aux firmes si l'innovation TPP a entraîné une modification majeure, mineure ou nulle dans l'utilisation des différents facteurs de production. Une autre possibilité serait de quantifier les changements, au moins approximativement.

247. Cet indicateur, qui donne une estimation très approximative de l'impact, peut se rapporter aux innovations TPP introduites au cours des trois dernières années, ou à une évaluation plus générale de la manière dont l'innovation a influé sur les indicateurs de performance.

\subsubsection{Réductions du coût moyen imputables aux innovations technologiques de procédé}

248. Il est suggéré que la question demande en premier lieu si les innovations technologiques de procédé introduites au cours des trois dernières années ont entraîné des réductions du coût moyen des produits fabriqués à l'aide de ces procédés. Si c'est le cas, on demandera ensuite de quantifier la réduction de coût.

\section{DIFFUSION DE L'INNOVATION}

249. Au chapitre 1, la diffusion a été définie comme étant la manière dont les innovations se répandent par l'intermédiaire des mécanismes du marché ou autrement, depuis la première réalisation en n'importe quel lieu du monde, jusqu'à d'autres pays et régions ainsi que d'autres industries/marchés et firmes. Afin de tracer un schéma des activités d'innovation et de brosser un panorama de certaines liaisons en jeu, ainsi que du niveau de diffusion des technologies avancées, il est proposé d'aborder les thèmes ciaprès. 


\subsection{Secteurs utilisateurs}

250. Théoriquement, les innovations pourraient être classées selon trois critères :

- le secteur de la principale activité économique du producteur ;

- le groupe technologique (groupe de produits) auquel appartient l'innovation ;

- le secteur probable d'utilisation.

251. Le premier critère est présenté de façon plus complète au chapitre 4 où sont traitées les classifications.

252. Il est possible de demander aux répondants de recenser le groupe technologique ou groupe de produits correspondant à leur innovation la plus importante (voir annexe 1).

253. Le troisième point peut être traité en demandant aux entreprises d'indiquer les proportions de ventes imputables aux produits technologiquement nouveaux ou améliorés en fonction du secteur de l'activité économique principale de leur(s) principal(aux) client(s) pour ces innovations technologiques de produit. La même question peut également être posée en ce qui concerne l'innovation (ou les innovations) la plus importante pour la firme (voir annexe 1).

254. Cependant, les réponses de certaines entreprises ou industries dont une forte proportion de ventes est effectuée par le biais de grossistes ne seront guère utiles pour faire ressortir le schéma de diffusion.

\subsection{Enquêtes sur l'utilisation de technologies avancées dans les procédés de fabrication}

255. Quelques pays ont mené des enquêtes sur l'utilisation de certaines technologies de pointe dans le secteur manufacturier et, dans un cas, dans le secteur des services également. Ces enquêtes décrivent un aspect important de la diffusion de l'innovation, à savoir la mesure dans laquelle les innovations sous forme de technologies nouvelles incorporées sont utilisées pour la production. Des enquêtes spécialisées visant le secteur manufacturier, principalement axées sur les applications de la micro-électronique, ont aussi été réalisées à un moment ou à un autre par de nombreux pays de l'OCDE.

256. Dans les enquêtes sur les techniques de fabrication, des renseignements étaient demandés sur l'utilisation, l'utilisation prévue et la non-utilisation de certaines technologies particulières. Elles ont montré que des enquêtes sur l'utilisation de la technologie peuvent être aisément réalisées et analysées, et qu'elles se prêtent à des comparaisons internationales. Elles peuvent également être conçues pour des industries spécifiques.

257. Le problème est d'établir une liste de technologies de pointe qui sont reconnues par l'industrie et qui ne revêtent pas un caractère tellement avancé qu'elles ne sont pas du tout utilisées. Ces technologies doivent être suffisamment utilisées pour que les statistiques relatives à leur utilisation effective et projetée dans une industrie fournissent des renseignements utiles aux décideurs. Les technologies devraient être définies de manière précise car une question portant sur des catégories de technologies trop générales, par exemple la biotechnologie ou la technologie de l'information, n'apporterait probablement pas d'informations très utiles. 
258. Une autre source de difficultés tient aux démarches en vue de parvenir à la comparabilité au plan international. Ces démarches comportent trois volets : l'établissement de la liste de technologies ; l'option entre soit une concordance convenue entre les classifications industrielles utilisées, soit le recours à une classification internationale agréée des branches d'activités; et le recours à un critère commun de couverture.

259. Il est possible de rattacher l'utilisation effective et prévue de technologies à d'autres questions liées à l'innovation. Des questions pour savoir si la technologie utilisée a été modifiée pour améliorer la productivité ou pour faciliter l'utilisation de la technologie, donnent des aperçus sur la propension à innover au niveau de l'atelier.

260. L'innovation dans la pratique de la gestion peut être rattachée à l'utilisation de la technologie. Dans le secteur manufacturier par exemple, une entreprise approvisionnant un client qui veut être livré "juste à temps", peut souhaiter améliorer son contrôle et son assurance qualité afin de réduire le taux de rejet des pièces produites. Dans le cadre de l'amélioration de la qualité, l'entreprise peut adopter un contrôle statistique du procédé et, en conséquence, utiliser des capteurs automatisés dans son procédé de fabrication. L'entreprise cliente peut utiliser un système automatisé de contrôle et de saisie des données et tous deux, le fournisseur et le client, peuvent être reliés par un réseau informatique

261. Les obstacles à l'innovation peuvent aussi être dépistés dans les enquêtes sur l'utilisation de la technologie, car on peut poser des questions sur la possibilité de disposer d'un personnel hautement qualifié et compétent pour mettre en oeuvre ces technologies nouvelles, ainsi que des fonds pour acheter la technologie et former des travailleurs.

262. Ces enquêtes sont considérées comme un moyen relativement simple d'obtenir des informations sur la diffusion de l'innovation, qui présentent un intérêt pour l'action gouvernementale. Quoiqu'elles puissent être intégrées aux enquêtes sur l'innovation, elles peuvent aussi présenter une utilité en tant que source indépendante d'informations statistiques que l'on peut reproduire et qui sont comparables au plan international, intéressant la politique dans le domaine de l'industrie et des échanges.

263. Il convient d'encourager les enquêtes sur l'utilisation de la technologie et, lorsque cela deviendra opportun, de les intégrer dans le contexte plus large des enquêtes sur l'innovation.

\section{QUESTIONS SPECIALES}

264. Un certain nombre d'autres sujets intéressant le processus d'innovation sont abordés dans la présente section : des questions sur la R-D qui ne figurent pas dans le Manuel de Frascati (et qui, de ce fait, ne sont généralement pas incluses dans les enquêtes sur la R-D) et des questions sur le dépôt de brevets ainsi que sur l'acquisition/diffusion de technologie.

\subsection{Questions spéciales sur la R-D}

265. Toutes les enquêtes sur l'innovation qui ont été réalisées à ce jour recoupent dans une certaines mesures les enquêtes sur la R-D (voir chapitre 7, section 2); les dépenses de R-D, par exemple, sont incluses dans les deux types d'enquêtes. Dans certains cas, il existe aussi d'autres sujets communs. Il se peut que ce chevauchement soit inévitable, car les organismes qui sont chargés des enquêtes sur l'innovation n'ont pas forcément accès aux données au niveau de l'entreprise obtenues par les enquêtes sur la R-D. On obtient ainsi des éclaircissements supplémentaires sur la R-D : presque toutes les enquêtes 
sur l'innovation exécutées jusqu'ici ont montré que les entreprises qui font état d'activités de R-D dans les enquêtes sur l'innovation sont beaucoup plus nombreuses que celles qui sont incluses dans les enquêtes sur la R-D. Cela pourrait tenir au fait que certains pays excluent de leurs statistiques les activités de R-D occasionnelles ou informelles, ou bien à ce que la complexité du questionnaire sur la R-D décourage peut-être les petites entreprises d'y répondre; il est possible aussi que cela s'explique parce que les enquêtes couvrent des populations statistiques différentes. Les entreprises incluses dans les enquêtes sur l'innovation, mais pas dans les enquêtes sur la R-D, sont généralement des PME.

266. Partant de l'hypothèse qu'au moins dans la plupart des pays, les enquêtes sur l'innovation seront dissociées des enquêtes sur la R-D, quelques questions sur la R-D que l'on pourrait inclure dans des enquêtes sur l'innovation sont recommandées ci-après. Dans de nombreux pays, ces questions pourraient aussi être incluses dans des enquêtes sur la R-D. Toutes les questions relatives à la R-D devraient être strictement conformes aux définitions et classifications du Manuel de Frascati.

267. Il est recommandé de demander des données sur les dépenses et le personnel de R-D, sauf si ces données sont disponibles dans d'autres enquêtes ou sources connexes relatives à la R-D. La question sur les dépenses de R-D recoupe celle sur les dépenses d'innovation, ce qui pourrait être une source de difficulté. En outre, il est suggéré de demander aux entreprises si leurs activités de R-D sont menées sur une base permanente ou occasionnelle. La répartition des dépenses de R-D entre R-D orientée vers des produits et $\mathrm{R}-\mathrm{D}$ orientée vers des procédés pourrait également être demandée.

268. Une autre question importante concerne la coopération en matière de R-D avec d'autres firmes, des instituts, des universités, à la fois dans le pays considéré et dans d'autres pays ou groupes de pays (coopération transnationale).

269. Il est recommandé d'inclure dans les enquêtes sur l'innovation une question concernant la coopération en matière de R-D en fonction des partenaires et des groupes de pays.

\subsection{Questions relatives aux brevets et à l'appropriabilité des innovations}

270. Les données sur les brevets, qu'il s'agisse des demandes ou des délivrances, ne sont pas des indicateurs des outputs de l'innovation, mais des indicateurs d'inventions, n'aboutissant pas nécessairement à des innovations. Néanmoins, des questions concernant le dépôt de brevets sont indispensables à une meilleure connaissance du processus d'innovation. Les séries générales de base sont, bien entendu, le nombre de dépôts et de délivrances de brevets par firme, disponibles auprès des diverses banques de données nationales et internationales. Des questions sur les brevets ont également été incluses dans les enquêtes sur la R-D ou sur l'innovation dans un certain nombre de pays.

271. Il est suggéré qu'il conviendrait de demander aux entreprises d'évaluer l'efficacité des diverses méthodes utilisées pour maintenir et accroître la compétitivité des innovations introduites au cours des trois dernières années. Ces méthodes pourraient être :

- les brevets ;

- le dépôt du modèle ;

- le secret;

- la complexité de la conception du produit ; 
- le fait d'avoir un temps d'avance sur les concurrents.

\subsection{Questions sur l'acquisition et/ou la diffusion de technologies}

272. Les enquêtes sur l'innovation comprennent les questions relatives à la balance des paiements technologiques (BPT) qui se situent à deux niveaux.

273. La façon la plus ambitieuse d'aborder cet aspect consiste à poser des questions sur les dépenses et les recettes afférentes aux brevets, aux licences, au savoir-faire, à l'assistance technique et à d'autres sortes d'acquisitions ou de ventes de technologies.

274. Dans l'autre démarche, aucune donnée monétaire n'est recueillie et on se contente de demander si la firme a acquis ou vendu des technologies sur le marché intérieur ou étranger.

275. La méthodologie appliquée en l'occurrence est celle décrite dans le Manuel sur la BPT de l'OCDE. Cependant, il n'est pas certain que l'on puisse demander des informations détaillées sur la BPT dans le cadre des enquêtes sur l'innovation : il est probablement préférable de réserver cette question à une enquête distincte. La méthode la moins ambitieuse est donc recommandée pour les enquêtes sur l'innovation.

276. Pour se faire une idée des relations entre l'acquisition de technologie, l'innovation et la vente de technologie, il est recommandé que l'enquête sur l'innovation demande au moins si la firme a acquis ou vendu de la technologie sur le marché intérieur ou étranger (données si possible ventilées par région) ou si elle a vendu de la technologie au marché intérieur ou étranger (avec une subdivision analogue). Ces informations devraient si possible être en outre ventilées selon le type de transaction (brevets, inventions non brevetées, licences, savoir-faire, marques de fabrique, services à contenu technologique, recours à des services de conseil, acquisition/transfert de technologie par le biais de l'achat/vente d'une firme, par le biais de l'achat/vente d'équipements, mobilité du personnel qualifié, etc.). 


\section{Chapitre 6}

\section{MESURE DES DÉPENSES D'INNOVATION}

277. Mesurer le coût total des activités d'innovation TPP dans les firmes et les industries est l'un des principaux buts des enquêtes sur l'innovation. Comme il est précisé dans le Manuel de Frascati, la R-D ne constitue que l'une des étapes du processus d'innovation. Les dépenses de R-D ne représentent donc qu'une partie de l'input financier. L'examen des dépenses pour l'ensemble des aspects de l'innovation TPP pourrait permettre un calcul plus rationnel du rendement des investissements dans ce domaine.

278. La dépense d'innovation TPP englobe l'ensemble des dépenses liées aux opérations scientifiques, technologiques, commerciales, financières et organisationnelles qui sont destinées à aboutir ou qui aboutissent effectivement à la mise en oeuvre de produits et procédés technologiquement nouveaux ou améliorés.

\section{MÉTHODE DE MESURE}

279. Bien que les principes directeurs actuels soient principalement axés sur l'approche sujet, dans les enquêtes sur l'innovation, les questions concernant les dépenses peuvent être présentées de deux manières :

$\Rightarrow$ Dépenses totales consacrées aux activités d'innovation pour l'entreprise au cours d'une année donnée (= approche sujet ou approche budget innovation).

$\Rightarrow$ Dépenses totales encourues pour les innovations accomplies au cours d'une année donnée ou pendant une période donnée, quelle que soit l'année où ces dépenses interviennent effectivement (= approche objet).

280. Il existe une différence fondamentale entre ces deux méthodes, aussi les résultats obtenus sont-ils différents. Ces deux approches ayant été utilisées dans un certain nombre d'enquêtes sur l'innovation, il semble utile de préciser leurs relations.

281. L'approche sujet porte sur les dépenses encourues pour l'ensemble des activités d'innovation TPP accomplies, potentielles ou avortées, telles qu'elles sont définies au chapitre 3. A cet égard, elle s'inscrit dans le prolongement direct de la mesure classique de la R-D. La fraction effective de R-D correspond aux dépenses couvertes par les enquêtes sur la R-D selon le Manuel de Frascati: elle englobe donc des dépenses de R-D qui ne sont pas directement liées à un projet d'innovation particulier. Peu nombreuses sont les firmes qui conservent des relevés distincts des autres dépenses d'innovation TPP, mais l'expérience a montré qu'il leur est tout à fait possible de fournir des estimations acceptables de cette partie hors R-D. 
282. Habituellement, les firmes innovantes poursuivent plusieurs projets d'innovation en même temps, et ces projets peuvent faire appel à des ressources assez différentes et s'étaler sur des périodes variables. Les grandes entreprises mènent généralement plus de projets d'innovation que les petites. En collectant des données sur le ou les principaux projets uniquement, on risque de manquer un montant des dépenses totales d'innovation beaucoup plus important pour les grandes entreprises que pour les petites. Ce phénomène influera aussi sur les dépenses d'innovation à l'échelle des secteurs d'activités, selon le degré de concentration sectorielle. L'approche sujet permet donc plus aisément d'obtenir une comparabilité internationale et une comparabilité entre secteurs d'activités et entreprises classées suivant leur taille.

283. L'approche sujet présente d'autres avantages, à savoir une plus grande comparabilité des données sur les dépenses d'innovation avec les données provenant des comptes nationaux, et une comparabilité entre les firmes novatrices et celles qui ne le sont pas. Il existe par ailleurs une relation clairement définie entre l'unité de mesure et la population de toutes les firmes, ce qui est l'objectif des analyses fondées sur les données recueillies.

284. Les inconvénients de cette approche tiennent, d'une part, au manque de correspondance entre l'effort d'innovation et son incidence sur les ventes et, d'autre part, à la difficulté de lier les résultats à des caractéristiques de l'innovation comme la durée du cycle de vie du produit, le temps nécessaire à la mise au point définitive, les conditions d'acquisition de droits, etc. Par ailleurs, il n'existe pas toujours de lien étroit entre le projet d'innovation et l'innovation qui est introduite sur le marché. Cette dernière peut être le résultat de divers projets, et un projet d'innovation peut être à l'origine de plusieurs innovations. On se heurte également à de graves problèmes de mesure lorsqu'il s'agit de déterminer les diverses sources de financement des dépenses d'innovation TPP.

285. Dans l'approche objet, la somme indiquée comprend les dépenses totales consacrées à des innovations TPP ou à la (les) principale(s) innovation(s) qui ont été accomplies au cours d'une période donnée. Elle exclut les dépenses afférentes à des projets d'innovation TPP qui ont avorté ou sont en cours, et les dépenses générales de R-D qui ne sont pas liées à un produit particulier ou à l'application d'un procédé spécifique. Cette approche semble particulièrement appropriée pour les enquêtes sur l'innovation qui sont faites à partir d'un ensemble d'innovations TPP réussies ou d'innovations TPP qui ont été accomplies. Mais elle pourrait être également utilisée dans les enquêtes sur les activités d'innovation TPP des firmes en général.

286. Les principaux avantages de cette approche tiennent à ce qu'elle permet de définir plus précisément les liens avec les résultats du processus d'innovation. Si l'enquête se situe au niveau d'une ou plusieurs innovations TPP, il est plus facile de relier les dépenses à des caractéristiques de l'innovation comme le temps nécessaire à la mise au point définitive, les conditions d'appropriation, la durée du cycle de vie du produit, le rôle des projets publics de R-D, ainsi que certains aspects de la diffusion des technologies. En outre, l'approche objet permet d'analyser la relation entre les activités d'innovation TPP ayant abouti et leur incidence sur les résultats économiques. Ces avantages sont cependant limités, car une innovation accomplie peut être le résultat de divers projets et, réciproquement, un projet d'innovation peut être à l'origine de plusieurs innovations. Par ailleurs, l'utilisation de cette approche atténue les problèmes de mesure soulevés par une ventilation détaillée des sources de financement des projets d'innovation TPP.

287. Avec l'approche objet, les entreprises devront reprendre la comptabilité financière des exercices précédents pour pouvoir fournir des chiffres précis, ce qui risque de soulever des difficultés. Cette méthode suppose en outre que les entreprises possèdent des informations sur les dépenses d'innovation au niveau des projets, ce qui est rarement le cas. Un autre problème tient à la définition du critère de détermination du "projet principal" (ou des projets principaux) qui varie parfois même à l'intérieur des 
entreprises, et peut donc également varier selon les entreprises, les secteurs et les pays. Il s'agit là d'un obstacle à la comparabilité internationale et à la comparabilité entre entreprises et industries.

288. Dans les enquêtes sur l'innovation fondées sur l'approche sujet, il est possible d'intégrer certains éléments de l'approche objet afin d'obtenir des détails supplémentaires sur le processus d'innovation. Dans ce cas, il importe de définir précisément dans le questionnaire les liens entre les rubriques relevant de chacune des deux approches.

289. Les enquêtes sur l'innovation fondées sur l'approche sujet peuvent permettre d'obtenir des informations sur le niveau auquel se situent la ou les principales innovations pour une année donnée. Les dépenses d'innovation permettent de faire le lien entre l'approche sujet et l'approche objet: l'approche objet permet de recueillir des informations sur l'ensemble des dépenses d'innovation pour le principal projet (ou les principaux projets) d'innovation et l'approche sujet d'obtenir des renseignements sur le budget innovation pour une année donnée. Ainsi est-il possible de relier les données collectées au niveau d'une innovation, ou des principales innovations (exemple : temps nécessaire pour parvenir à la commercialisation, temps de retour escompté) à l'ensemble des activités d'innovation d'une firme. Une fois encore, le problème clé semble être celui de la définition du critère de détermination du "projet principal" (ou des projets principaux). On trouvera plus de détails à l'annexe 1, intitulée "Utilisation de l'approche objet pour la collecte de données sur l'innovation".

290. Compte tenu des avantages et inconvénients des deux méthodes, l'approche sujet est recommandée pour déclarer les dépenses d'innovation TPP. Ce chapitre traite principalement de cette approche, encore que la plupart des définitions et des conseils s'appliquent aussi plus ou moins à l'approche objet.

\section{VENTILATIONS SUGGÉRÉES}

291. Les dépenses totales consacrées aux activités d'innovation TPP comprennent les dépenses courantes et les dépenses en capital encourues pour les types d'activités d'innovation définis au chapitre 3 .

292. Il est recommandé de recueillir des données sur la ventilation des dépenses totales d'innovation TPP par type d'activité d'innovation TPP ainsi que par type de dépenses (dépenses courantes par opposition aux dépenses en capital). Compte tenu de l'importance accordée aux contraintes de financement dans les débats politiques, il est également souhaitable de disposer de données sur les dépenses d'innovation TPP ventilées par source de financement.

\subsection{Méthode agrégative ou désagrégative}

293. On distingue en principe deux méthodes pour recueillir des données sur les dépenses d'innovation et opérer des ventilations détaillées par type d'activité d'innovation. La méthode agrégative consiste à demander le montant des dépenses d'innovation pour chaque type d'activité d'innovation et à en faire la somme pour obtenir la dépense totale d'innovation de la firme. L'approche désagrégative, à l'inverse, consiste à demander la somme de l'ensemble des dépenses d'innovation, puis la ventilation de cette dépense par type d'activité. La méthode agrégative est recommandée car elle donne des résultats plus fiables. Cependant, comme les chiffres correspondant à une ventilation par type d'activité ne sont pas tous aussi faciles à obtenir dans les firmes (et que les données correspondant à certains postes de dépenses ne sont parfois pas du tout disponibles dans certaines firmes), une approche désagrégative permettra peutêtre à certaines entreprises de répondre plus facilement et pourrait être utilisée pour estimer la dépense d'innovation par type de dépense ou par type d'activité d'innovation. 
294. Dans la mesure du possible, les réponses relatives à diverses ventilations des dépenses d'innovation TPP devraient être exprimées en termes monétaires. Néanmoins, si cela paraît trop difficile, on pourrait envisager de demander d'indiquer la somme totale accompagnée d'estimations des ventilations en pourcentage correspondant aux différentes rubriques. L'expérience récente laisse supposer que le taux de non-réponse à ces questions diminue si les entreprises ont le choix d'exprimer leur réponse en termes monétaires ou en pourcentage.

\subsection{Ventilation par type de dépense}

295. Les dépenses consacrées à des activités d'innovation TPP devraient, si possible, être ventilées en dépenses courantes et dépenses en capital. Cette démarche est de la plus haute importance si l'on veut comparer les données aux chiffres des investissements immatériels, avec lesquels les dépenses d'innovation sont parfois confondues (voir plus loin la section 2.2.1).

296. Les dépenses courantes d'innovation comprennent les coûts de main-d'oeuvre et les autres dépenses courantes:

- Les coûts de main-d'oeuvre sont constitués par les salaires et traitements annuels, ainsi que tous les coûts annexes extra-salariaux tels que primes, congés payés, cotisations aux fonds de pension et autres cotisations de sécurité sociale, prélèvements sur les salaires, etc. Les coûts de main-d'oeuvre des personnes qui ne participent pas aux activités d'innovation TPP (par exemple du personnel de sécurité et de maintenance) devraient être exclus et comptabilisés avec les autres dépenses courantes ;

- Les autres dépenses courantes sont constituées par les frais encourus pour l'achat de matériaux, fournitures, services et équipements qui ne font pas partie des dépenses en capital, en vue de soutenir les activités d'innovation TPP menées par la firme au cours d'une année donnée.

297. Les dépenses en capital consacrées à l'innovation sont les dépenses annuelles brutes afférentes aux éléments de capital fixe utilisés pour les activités d'innovation TPP de la firme. Elles doivent être déclarées dans leur intégralité pour la période au cours de laquelle elles ont été engagées et non pas indiquées comme un élément d'amortissement. Elles comprennent les dépenses consacrées aux terrains et bâtiments, aux instruments et équipements et, conformément au Système de comptabilité nationale (SCN) révisé, au logiciel, qui est un élément des investissements immatériels et dont l'acquisition est considérée comme de la formation de capital :

- dans la catégorie "terrains et bâtiments" entrent l'acquisition de terrains et de bâtiments destinés à des activités d'innovation TPP, mais également les améliorations, modifications et réparations importantes de ces terrains et bâtiments ;

- dans la catégorie "instruments et équipements" entrent les principaux instruments et équipements acquis aux fins des activités d'innovation TPP de la firme ;

- dans la catégorie "logiciel", conformément au SCN révisé, entrent les logiciels système et logiciels d'application (programmes, descriptions, documentation, etc.) utilisés dans les activités d'innovation TPP de la firme. Sont inclus les logiciels achetés et ceux produits pour compte propre (à condition que la dépense soit suffisamment importante) destinés aux activités d'innovation TPP. Les dépenses importantes pour l'achat, la mise au point ou l'extension de bases de données destinées à être utilisées pendant une durée supérieure à un an dans le cadre des activités d'innovation TPP sont également incluses, que ces bases soient commercialisées ou non. 
298. Toutes les provisions pour amortissement des bâtiments, installations et équipements, qu'elles soient réelles ou imputées, devront être exclues de la mesure des dépenses intra-muros.

299. L'innovation TPP, en particulier l'innovation technologique de procédé, implique souvent l'installation de nouvelles machines et de nouveaux équipements. Trois cas peuvent se présenter :

- l'installation de machines et d'équipements aux performances technologiques améliorées (c'est-àdire qui améliorent les méthodes de production de la firme) (voir chapitre 3 , section 5.2.2) constitue une innovation technologique de procédé. Le coût de l'équipement doit figurer dans les dépenses en capital consacrées à l'innovation TPP. D'un autre point de vue, il s'agit d'un élément entrant dans les investissements bruts en capital fixe de la firme ; la méthode de classification retenue ici vise toutefois à déterminer les dépenses se rapportant à la diffusion des innovations ;

- l'installation de machines et d'équipements dont les performances technologiques n'ont pas été améliorées (c'est-à-dire qui n'améliorent pas les méthodes de production), qui est nécessaire pour fabriquer un produit technologiquement nouveau (par exemple, une nouvelle machine à mouler ou à emballer), ne constitue pas une innovation technologique de procédé. Le coût de l'équipement figure toutefois dans les dépenses en capital consacrées à l'innovation TPP ;

- les autres achats de machines et d'équipements ne sont pas considérés comme des innovations technologiques de procédé et ne doivent pas figurer dans les dépenses d'innovation TPP. Par exemple, le fait d'augmenter la capacité de production en rajoutant des machines d'un modèle déjà utilisé ou même en remplaçant des machines par des versions plus récentes du même modèle ne constitue pas une innovation TPP.

300. Les entreprises éprouvent souvent de graves difficultés à fournir des estimations fiables des dépenses en capital destinées aux activités d'innovation TPP. Pour les y aider, il est proposé de recueillir aussi des données sur l'ensemble des dépenses en capital (y compris les dépenses qui ne sont pas liées à des activités d'innovation TPP). Ceci permettra également de vérifier la fiabilité des données sur les dépenses d'innovation TPP et de se faire une idée de la relation entre les dépenses d'innovation TPP et les investissements matériels.

\subsubsection{Relation entre les investissements immatériels et les dépenses d'innovation TPP}

301. Par investissements immatériels, on entend toutes les dépenses courantes visant le développement de la firme dont on espère un bénéfice au-delà de l'année au cours de laquelle elles ont été effectuées. Il n'existe pas de définition normalisée de ces investissements, mais on considère en général qu'ils couvrent, outre les dépenses courantes de R-D, les dépenses de commercialisation exceptionnelles, les dépenses de formation, les dépenses de logiciel et d'autres postes analogues.

302. Les dépenses courantes consacrées aux innovations TPP sont manifestement un élément des investissements immatériels. Les investissements immatériels comprennent des éléments qui ne font pas partie des dépenses courantes d'innovation TPP. Par exemple, seule la formation liée à l'introduction de produits et procédés technologiquement nouveaux ou améliorés entre dans la catégorie des dépenses d'innovation TPP, alors que les investissements immatériels englobent l'ensemble des dépenses de formation de la firme. Les frais de commercialisation liés à l'introduction de produits et procédés technologiquement nouveaux ou améliorés entrent dans les dépenses d'innovation TPP. Les investissements immatériels comprennent en outre les dépenses de commercialisation de caractère général 
(destinées, par exemple, à améliorer l'image de la firme, ou à ouvrir de nouveaux marchés, sans lien direct avec des produits et des procédés technologiquement nouveaux ou améliorés).

303. Par ailleurs, les dépenses d'innovation TPP comprennent des investissements matériels, tels que les dépenses en capital relatives à la $\mathrm{R}-\mathrm{D}$ et l'acquisition de machines et équipements nouveaux en relation avec les innovations TPP.

\subsection{Ventilation par type d'activités d'innovation TPP}

304. Les descriptions des postes de dépenses à inclure au titre des diverses catégories d'activités d'innovation TPP sont fondées sur les définitions des activités d'innovation TPP présentées au chapitre 3, section 5 .

305. La ventilation suivante devrait être considérée comme un principe directeur général applicable au secteur manufacturier et aux services. Pour le secteur des services, tous les éléments ne semblent pas revêtir d'importance et certains devraient être omis. Par exemple, l'expérience récente laisse penser que des rubriques de dépenses comme la conception, l'ingénierie industrielle et les essais de production peuvent être sans objet pour le secteur des services. A l'inverse, une activité comme le logiciel, qui touche toutes les activités TPP, sera peut-être plus facile à identifier et pourra présenter de l'intérêt pour les services.

306. Afin de faciliter la comparaison avec les dépenses de R-D, il est recommandé de recueillir les informations concernant la ventilation par type d'activités d'innovation TPP pour le total des dépenses d'innovation TPP (dépenses courantes et en capital). La ventilation suivante est recommandée :

- dépenses de R-D ;

- dépenses encourues pour l'acquisition de technologie et de savoir-faire non incorporés ;

- dépenses encourues pour l'acquisition de technologie incorporée ;

- dépenses encourues pour l'outillage, l'ingénierie industrielle, les études de conception industrielle et le démarrage de la production, y compris d'autres dépenses relatives aux installations pilotes et aux prototypes qui ne sont pas déjà incluses dans la R-D ;

- dépenses de formation liées à des activités d'innovations TPP ;

- commercialisation de produits technologiquement nouveaux ou améliorés.

\subsubsection{Dépenses de $R-D$}

307. Cette rubrique comprend les dépenses totales intra-muros et extra-muros consacrées à la R-D, telles qu'elles sont définies dans le Manuel de Frascati (voir aussi le chapitre 3, section 5.2.1.a). Si les dépenses intra-muros et extra-muros de R-D sont évaluées séparément, cela facilitera la comparaison avec les données tirées d'enquêtes sur la R-D. 
308. Dépenses intra-muros de R-D : Cette rubrique comprend l'ensemble des dépenses intra-muros consacrées à la R-D au sein de la firme, telles qu'elles sont définies dans le Manuel de Frascati et indiquées dans les enquêtes sur la R-D. Dans la plupart des cas, tous ces travaux de R-D ont pour but de contribuer à l'introduction de produits et procédés technologiquement nouveaux ou améliorés dans la firme concernée. Toutefois, lorsqu'une firme mène des travaux de R-D exclusivement à titre de service pour le compte d'une autre firme (ou d'un organisme public) et uniquement en vue de contribuer à des activités d'innovation de cette dernière (ou de ce dernier), il convient d'essayer d'identifier les fonds en question, de manière à pouvoir les exclure pour éviter un double comptage lorsqu'on établit la somme des dépenses totales (intra-muros et extra-muros) pour l'ensemble des industries. Cette rubrique couvre en outre la R-D qui ne vise pas à élaborer des produits ou procédés nouveaux particuliers, mais à élargir la base de connaissances d'une firme.

309. Dépenses extra-muros de R-D : Cette rubrique comprend l'acquisition de services de R-D.

\subsubsection{Dépenses encourues pour l'acquisition de technologie et de savoir-faire non incorporés}

310. Cette rubrique comprend les dépenses relatives à l'acquisition de technologie non incorporée, telle que définie au chapitre 3, section 5.2.1.b. Les dépenses consacrées aux services de R-D sont exclues.

\subsubsection{Dépenses encourues pour l'acquisition de technologie incorporée}

311. Cette rubrique comprend les dépenses relatives à l'acquisition de machines et d'équipements aux performances technologiques améliorées, y compris les logiciels importants, directement liés à des procédés technologiquement nouveaux ou améliorés tels que définis au chapitre 3, section 5.2.1.c.

2.3.4 Dépenses encourues pour l'outillage, l'ingénierie industrielle, les études de conception industrielle et le démarrage de la production (y compris les autres dépenses relatives aux installations pilotes et aux prototypes qui ne sont pas déjà incluses dans la $R$-D)

312. Cette rubrique comprend principalement :

- les dépenses encourues pour l'outillage et l'ingénierie industrielle tels que définis au chapitre 3, section 5.2.2.a., et notamment pour le développement de l'organisation en liaison avec le démarrage de la production ;

- les dépenses encourues pour la conception industrielle de produits ou procédés technologiquement nouveaux ou améliorés tels que définis au chapitre 3 , section 5.2.2.b, dans la mesure où elles ne sont pas déjà incluses dans les dépenses de R-D ;

- les dépenses encourues pour tester les produits et services technologiquement nouveaux ou améliorés (les essais de prototypes font partie de la R-D et ne figurent donc pas dans cette rubrique) ;

- les dépenses encourues pour l'acquisition d'autres équipements, tels que définis au chapitre 3, section 5.2.2.c, nécessaires à la mise en oeuvre des innovations TPP ;

- les dépenses encourues pour le démarrage de la production tel que défini au chapitre 3, section 5.2.2.d, à l'exception des dépenses relatives à la formation du personnel qu'il est proposé d'inclure dans une catégorie distincte ; 
- les dépenses afférentes aux essais de production et aux installations pilotes, si elles ne sont pas déjà incluses dans la R-D (les essais de production sont inclus dans la R-D si la production requiert des essais en vraie grandeur et donc de nouvelles études de conception et d'ingénierie ; les installations pilotes sont incluses dans la R-D si leur objectif principal est la R-D) ;

- les autres dépenses concernant les prototypes dans la mesure où elles ne sont pas déjà incluses dans la R-D ;

- les dépenses liées au respect des prescriptions réglementaires. Celles-ci peuvent englober l'homologation des produits pharmaceutiques, la conformité aux réglementations en matière d'environnement, et diverses normes et obligations (visant par exemple la protection de l'environnement).

\subsubsection{Dépenses de formation liées à des activités portant sur des innovations TPP}

313. Cette rubrique comprend principalement les dépenses de formation requises pour la réalisation de produits et procédés technologiquement nouveaux ou améliorés (la formation en vue d'autres activités ne doit pas être prise en compte, voir chapitre 3, section 5.3.2). En règle générale, l'apprentissage ne fait pas partie des dépenses d'innovation. Les dépenses d'innovation TPP englobent donc principalement la formation ultérieure, qui couvre différents types de formation liés à des innovations TPP. La mesure de la formation est examinée de façon plus détaillée dans les chapitres II et III du Manuel de l'OCDE sur les statistiques de formation qui doit paraître prochainement.

\subsubsection{Commercialisation de produits technologiquement nouveaux ou améliorés}

314. Cette rubrique comprend les dépenses encourues pour les activités liées au lancement de produits technologiquement nouveaux ou améliorés, tel que défini dans le chapitre 3, section 5.2.3.

\subsection{Problèmes de mesure}

315. Dans plusieurs enquêtes sur l'innovation des données sur la part de R-D aussi bien que sur la part hors R-D de l'ensemble des dépenses d'innovation ont été recueillies. Il s'est avéré que de nombreuses firmes ont rencontré des difficultés pour rendre compte de leurs dépenses d'innovation. Leur système de comptabilité ne leur permet habituellement pas d'obtenir directement les données correspondant aux rubriques hors R-D. Par conséquent, la question fondamentale n'est pas "quelles données collecter", mais "comment obtenir des données fiables" sur les dépenses d'innovation autres que les dépenses de R-D.

316. La qualité des données recueillies sur les dépenses d'innovation dépend fondamentalement de la conception du questionnaire. Des modifications mineures dans les définitions ou les explications fournies dans la partie du questionnaire traitant des dépenses d'innovation TPP, ainsi que dans la présentation du questionnaire lui-même ou l'ordre des questions ou rubriques, auront une incidence sur les informations recueillies.

317. Afin d'évaluer la fiabilité des réponses, il serait peut-être utile de demander aux entreprises d'indiquer le degré d'incertitude entachant les données en précisant si celles-ci sont tirées de comptes détaillés ou s'il s'agit d'estimations relativement précises ou approximatives. Bien que ce type de questions puisse augmenter la proportion des participants qui se contenteront de fournir des estimations grossières des chiffres, il se peut que le taux de réponse à l'enquête s'en trouve augmenté. 


\subsubsection{Limite entre les dépenses d'innovation de $R$-D et hors $R-D$}

318. Il est apparu dans les récentes enquêtes sur l'innovation que certaines entreprises éprouvaient des difficultés à faire la distinction entre les dépenses totales d'innovation et les dépenses de R-D, notamment à la frontière entre les dépenses de R-D et hors R-D. L'expérience récente a montré que, selon qu'ils étaient mesurés en tant que partie des dépenses totales d'innovation ou qu'ils faisaient l'objet d'une question distincte (question de type Frascati), les chiffres de dépenses de R-D ne coïncidaient pas, même si ces deux types de questions figuraient dans le même questionnaire. Ce phénomène résulte de l'utilisation de différentes méthodes de comptabilisation de la R-D au sein des entreprises ; elles ne sont parfois pas pleinement conformes à la définition des dépenses de R-D du Manuel de Frascati et incluent certaines activités hors R-D. Des explications détaillées et la présentation du questionnaire devraient néanmoins aider les entreprises à fournir des réponses cohérentes concernant la R-D. Ce problème est particulièrement sensible dans les industries où les activités novatrices sont, dans une large mesure, des activités de conception (par exemple la construction automobile).

319. Il convient de s'attacher à exclure les activités qui font partie du processus d'innovation, mais font rarement appel à la R-D (travaux concernant les brevets, octroi de licences, études de marché, démarrage de la fabrication, réorganisation des procédés, outillage, par exemple). Par ailleurs, certaines activités sont au moins en partie comptabilisées dans la R-D (les installations pilotes, les prototypes, la conception industrielle, la mise au point de procédés, par exemple).

320. Le critère fondamental qui permet de distinguer les activités de R-D des activités d'innovation hors R-D “est l'existence, au sein de la R-D, d'un élément de nouveauté non négligeable et la dissipation d'une incertitude scientifique et/ou technologique" (voir Manuel de Frascati, para. 79). D' après ce critère, "un projet particulier peut être considéré comme de la R-D s'il est entrepris dans un certain but, mais ne le sera pas s'il est entrepris dans un autre but" (Manuel de Frascati, para. 80).

321. Le Manuel de Frascati (para. 112) suggère d'utiliser la règle fondamentale établie par la Fondation nationale pour la science (NSF) des États-Unis comme critère général de distinction entre les activités de R-D et celles hors R-D :

"Si l'objectif principal des travaux est d'apporter de nouvelles améliorations techniques au produit ou au procédé, ils tombent alors sous la définition de la $R-D$. Si, au contraire, le produit ou le procédé ou l'approche est en grande partie "fixé" et si l'objectif principal est de trouver des débouchés, d'établir des plans de préproduction ou d'obtenir un système de production ou de contrôle harmonieux, il ne s'agit pas de R-D."

322. Il est recommandé que les principes directeurs du Manuel de Frascati énoncés aux paragraphes 111 à 132 soient appliqués aux enquêtes sur l'innovation. Dans un certain nombre de cas, les entreprises continueront, en particulier dans certains secteurs industriels, à éprouver des difficultés à distinguer, parmi leurs activités d'innovation, celles qui relèvent de la R-D de celles qui n'en relèvent pas.

\subsubsection{Autres difficultés}

323. Une ventilation entre dépenses intra-muros et dépenses extra-muros de chacune des catégories susmentionnées de types d'activité fournirait des informations utiles. Une telle ventilation n'est cependant pas réalisable dans le cas de la plupart des entreprises, aussi cette méthode n'est-elle pas recommandée ici.

324. En conséquence, il convient de se montrer particulièrement prudent dans le calcul de valeurs nationales ou sectorielles en faisant la somme des chiffres fournis par les entreprises, en raison des 
doubles comptages possibles. Pour évaluer approximativement l'importance du double comptage, il semblerait utile de savoir si les dépenses afférentes aux services extérieurs sont ou non incluses.

325. Les dépenses d'innovation TPP effectuées au cours d'une année donnée peuvent être parfois trompeuses. Les petites entreprises, en particulier, ne se livrent pas à des activités d'innovation TPP en continu. Recueillir des informations sur les dépenses d'innovation TPP pour une période couvrant plusieurs années permet donc d'obtenir des renseignements supplémentaires utiles sur les activités d'innovation TPP. Mais le manque de disponibilité des données au sein des entreprises constitue un obstacle sérieux à cette approche pluriannuelle.

326. Un moyen d'y remédier consisterait à demander en outre si les dépenses d'innovation des années précédentes ont été nettement supérieures ou nettement inférieures à celles mentionnées pour l'année concernée. De plus, pour permettre d'évaluer plus aisément l'évolution la plus récente des dépenses d'innovation, il semble utile de demander si l'on prévoit que ces dépenses augmenteront, diminueront ou se maintiendront au même niveau. Ceci semble plus particulièrement souhaitable dans une perspective de politique de l'innovation.

\subsection{Ventilation par source de financement}

327. Il importe de savoir quelles sont les sources de financement des dépenses d'innovation TPP afin, par exemple, d'évaluer le rôle de l'action gouvernementale et de l'internationalisation dans le processus d'innovation. Il est suggéré d'adopter la classification par source de financement suivante :

\section{Liste des sources de financement :}

- Fonds propres ;

- fonds provenant d'entreprises apparentées (filiales ou entreprises associées) ;

- fonds provenant d'autres entreprises commerciales ;

- fonds de l'État (prêts, subventions, etc.);

- financement provenant d'organismes supranationaux et internationaux (CE, etc.);

- Autres sources.

328. Il suffit, concernant divers aspects portant sur les questions de politique et d'analyse, de recueillir des informations sur la contribution ou non de chaque source de financement, plutôt que de chercher à obtenir une estimation, probablement imprécise, des montants (en termes monétaires ou en pourcentage) provenant de chacune de ces sources. La charge de travail que la réponse au questionnaire représente pour les entreprises s'en trouverait considérablement réduite, ce qui améliorerait le taux de réponse au questionnaire et réduirait le taux de non réponse à cette question.

329. Il est utile, pour évaluer le rôle des marchés publics dans le processus d'innovation, de savoir si une firme est ou non partie prenante à des marchés publics (régionaux, nationaux ou internationaux) ayant trait à des produits ou procédés novateurs. Cela pourrait remplacer utilement une ventilation détaillée par source de financement. 


\section{Chapitre 7}

\section{PROCÉDURES D'ENQUÊTE}

330. Pour la collecte et l'analyse des données sur l'innovation, il est essentiel d'appliquer correctement les méthodes statistiques. S'appuyant sur les connaissances théoriques, de même que sur l'expérience pratique acquise au cours de récentes enquêtes sur l'innovation menée au niveau national et international, le présent chapitre formule des recommandations concernant les éléments clés de la collecte et de l'analyse des données sur l'innovation.

331. En suivant ces recommandations, on aboutira généralement à des résultats comparables dans le temps et entre pays. Il se peut que des circonstances particulières obligent un pays à recourir à une autre méthodologie. Cela ne posera pas de problèmes dès lors que les résultats restent comparables.

\section{POPULATIONS}

332. Des activités d'innovation se rencontrent dans toutes les parties d'une économie: dans les industries manufacturières, le secteur des services, les administrations publiques, le secteur de la santé et même dans le secteur des ménages. Toutes les unités d'une économie qui correspondent ou qui pourraient correspondre au champ couvert restreint défini au chapitre 2 (unités innovantes ou non innovantes), sont susceptibles d'êtres prises en compte dans les enquêtes sur l'innovation.

333. En réalité, pour diverses raisons théoriques et pratiques, une enquête ne recouvrira pas toutes les unités possibles. Le concept d'innovation est encore imprécis dans certaines parties de l'économie, notamment en ce qui concerne les activités non axées sur le marché. Il est donc recommandé que les enquêtes sur l'innovation portent principalement sur les activités d'innovation dans des secteurs axés sur le marché. Ces secteurs devraient inclure les industries manufacturières, de même que les industries de services axées sur le marché. Tant que les connaissances relatives au activités d'innovation dans le secteur des services demeureront assez limitées, dans cette étape initiale d'élaboration de la méthodologie, il sera préférable de concentrer l'attention sur les industries de services à forte intensité technologique.

334. Les activités d'innovation TPP sont menées dans les unités de petite et moyenne importance aussi bien que dans les grandes unités. En théorie, les enquêtes sur l'innovation devraient donc comprendre des unités de toutes tailles. Cependant, pour des raisons pratiques, seules les unités employant au moins dix personnes devront être couvertes pour assurer la comparabilité internationale. Ce seuil peut être relevé pour des industries spécifiques et abaissé pour certains secteurs des services.

335. Toutes les unités dont les effectifs sont égaux ou supérieurs à dix, et appartenant à l'un des secteurs mentionnés ci-dessus, constituent la population cible des enquêtes sur l'innovation. La population cible inclut des unités innovantes et non innovantes, des unités qui exécutent de la R-D et des unités qui n'exécutent pas de R-D. 
336. En pratique, il est presque impossible de recenser et de contacter toutes les unités de la population cible quel que soit le type d'enquête. Ainsi, il se peut que le cadre sur lequel s'appuie l'enquête (un registre par exemple) comprenne des unités qui n'existent plus, ou des unités qui n'appartiennent plus à la population cible. En revanche, il se peut que des unités qui appartiennent en fait à la population cible ne figurent pas dans ce cadre. Les unités couvertes par la base de l'enquête constituent la population cadre.

337. Lors de la préparation d'une enquête, les populations cible et cadre devraient être aussi proches l'une de l'autre que possible. Les organismes menant les enquêtes sur l'innovation ne devraient ménager aucun effort possible afin de réduire les erreurs dues aux différences entre ces deux populations. Dans la plupart des cas, l'importance des erreurs serait inacceptable si la population cadre était définie sur la base des demandes de subventions à la R-D (hypothèse de base : seules les unités qui exécutent de la R-D mènent des activités d'innovation; problème supplémentaire : toutes les unités exécutant des travaux de R-D ne sollicitent pas des subventions), ou sur la base d'informations relatives à d'anciennes unités innovantes. Dans certains cas, la population cadre peut être identifiée au moyen d'une enquête ad hoc ou à l'aide d'enquêtes existantes.

\section{MÉTHODES D'ENQUÊTE}

\subsection{Recensement ou enquête par sondage}

338. Des données sur l'innovation peuvent être recueillies grâce à des recensements ou à des enquêtes par sondage. Dans la plupart des cas, les ressources étant limitées et étant donné la charge que représente la réponse, il sera exclu que l'enquête porte sur toute la population (recensement). Si l'on a recours à des enquêtes par sondage, il convient de sélectionner les unités sur la base d'une procédure aléatoire (enquêtes portant sur des échantillons aléatoires). Cet échantillon devrait être représentatif des caractéristiques fondamentales de la population cible, telles que l'industrie considérée ou la taille des unités.

339. Dans certains cas, le recours au recensement pourrait être inévitable. Il pourrait être juridiquement imposé que toutes les enquêtes de conjoncture revêtent la forme de recensements. En outre, lorsque la population cadre est plutôt restreinte (dans de petits pays, par exemple) et si des techniques d'échantillonnage plus complexes, telles que des techniques de stratification, sont proposées, un échantillonnage approprié pourrait faire que la taille de l'échantillon ne diffère guère de celle de la population cadre. En de pareils cas, il vaudrait peut-être la peine d'envisager un recensement.

\section{$2.2 \quad$ Enquête obligatoire ou facultative}

340. Les enquêtes sur l'innovation peuvent être obligatoires ou facultatives. Lorsqu'elles sont facultatives, il y a lieu de s'attendre à des taux de non-réponse plus élevés. De faibles taux de réponses pourraient se traduire par des nombres de réponses insuffisants pour pouvoir servir à une analyse plus poussée. S'agissant des enquêtes par sondage, cet effet pourrait, dans une certaine mesure, être compensé par l'application de taux de sondage plus élevés. Toutefois, cela ne résout pas le problème fondamental de la distorsion imputable au taux élevé de non-réponse.

341. Pour suivre l'évolution du processus d'innovation dans le temps, les enquêtes par panel (échantillon) offrent des possibilités particulières. Notamment, elles permettent aux analystes d'examiner les liens de causalité entre différentes variables dans le temps. Les enquêtes par panel exigent de 
sélectionner avec un soin particulier les unités et de faire preuve de vigilance lors du traitement des unités qui refusent de répondre, qui disparaissent ou qui sont nouvellement créées.

\subsection{La population cadre}

342. Pour toute enquête sur l'innovation, il est nécessaire de disposer d'un cadre comportant des informations élémentaires sur toutes les unités de la population cadre. Au minimum, il faut disposer des noms et des adresses de toutes les unités. Il est souhaitable d'avoir les numéros de téléphone et de télécopie. En outre, le cadre devrait inclure des informations sur des variables clés telles que le secteur d'activité, la taille de l'unité ou la région.

343. Un registre officiel et à jour des entreprises, établi à des fins statistiques, constitue le cadre idéal. De tels registres sont généralement tenus par les instituts nationaux de statistiques. D'autres registres peuvent être également utilisés, suivant leur qualité. Si ces registres servent de base à plusieurs enquêtes, telles l'enquête sur l'innovation, l'enquête sur la R-D et l'enquête générale de conjoncture, les informations recueillies dans l'enquête sur l'innovation peuvent ne porter que sur des aspects ayant spécifiquement trait à l'innovation. D'autres informations concernant, par exemple, la R-D ou des variables économiques générales, telles que les ventes, les exportations ou les investissements, peuvent être directement tirées des autres enquêtes fondées sur ces registres. Il est donc souhaitable de fonder différentes enquêtes sur un seul registre d'entreprises compilé à des fins statistiques. Si de tels liens entre enquêtes ne sont pas possibles, des données économiques générales et des données sur la R-D doivent également être recueillies lors des enquêtes sur l'innovation.

\section{$2.4 \quad$ Méthodes d'enquête et répondants appropriés}

344. Diverses méthodes peuvent être utilisées pour la collecte d'informations, notamment l'enquête par voie postale ou l'entretien personnel. Dès lors que les enquêtes sur l'innovation sont bien établies, l'échange automatisé de données entre les unités déclarantes et l'institut de sondage peut se révéler possible également.

345. Ces méthodes offrent chacune différents avantages et inconvénients. Les enquêtes par voie postale sont bien établies et relativement moins coûteuses, mais elles présentent des problèmes également. L'expérience montre que les questionnaires des enquêtes par voie postale doivent être extrêmement bien conçus afin d'obtenir des taux de réponse suffisants (pour davantage de détails, voir plus loin), et l'organisme qui mène l'enquête devrait encourager les répondants à téléphoner pour élucider les questions et solliciter de l'aide. Il faut, généralement, plusieurs rappels pour amener le taux de réponse à un niveau acceptable. Une autre difficulté tient au fait que les rappels peuvent donner lieu à des réponses différentes de la part de répondants différents dans la même entreprise. Des mesures supplémentaires peuvent être prises afin de relever encore les taux de réponse : joindre une lettre de couverture signée par le ministre responsable, envoyer les résultats essentiels des enquêtes précédentes sur l'innovation (s'il en existe), ou promettre d'envoyer aux répondants les principales conclusions de l'enquête en cours.

346. L'entretien personnel permet d'éviter la plupart des problèmes rencontrés dans le cas d'enquêtes par voie postale. La qualité des résultats devrait être nettement supérieure. Les taux de non-réponse globale et partielle devraient fortement diminuer, de sorte qu'il faudra contacter un nombre beaucoup plus restreint d'unités pour atteindre la même qualité. En dépit de ces avantages évidents, il n'est pas recommandé de recourir de façon généralisée à cette méthode, car les coûts en sont encore fort élevés et, dans la plupart des cas, trop élevés. 
347. La meilleure solution serait peut-être d'associer les avantages des méthodes par voie postale et ceux des entretiens tout en évitant leurs inconvénients. Les techniques CATI vont dans ce sens, de même que la méthode canadienne dans laquelle des questionnaires personnalisés sont conçus pour chaque unité, sur la base des informations obtenues grâce à des contacts personnels (par téléphone, par exemple) avec le répondant le mieux approprié dans l'unité en question. Ces questionnaires propres à chaque unité sont alors envoyés par voie postale.

348. Dans les enquêtes sur l'innovation, il importe tout particulièrement de choisir le répondant le mieux approprié dans les unités, car les questions sont éminemment spécialisées et quelques personnes seulement dans ces unités peuvent y répondre; or, en général, ce ne sont pas celles qui répondent habituellement aux autres questionnaires statistiques. Dans les petites unités, le directeur général est souvent un bon répondant. Le directeur chargé de la technologie pourrait être le mieux placé pour répondre aux questions dans les grandes unités. Il faudrait souvent faire appel à plusieurs personnes, mais l'une d'elles devra être chargée de coordonner les réponses. Un effort particulier en vue d'identifier les répondants est fortement recommandé avant de lancer la collecte de données. Cela contribuera largement au succès de l'enquête, mais risque de s'avérer difficile dans la pratique. Il est important que le partenaire dans l'unité ait en son pouvoir de décider de la participation à l'enquête (si celle-ci est facultative) et de collecter les données nécessaires concernant l'unité.

\subsection{Le questionnaire}

349. Toutes les techniques de collecte de données se fondent, au moins dans une certaine mesure, sur un questionnaire. Certaines règles de base devraient être suivies lors de la conception du questionnaire destiné à une enquête sur l'innovation. Un soin particulier est nécessaire dans le cas des enquêtes par voie postale. Chaque questionnaire devrait être testé avant d'être utilisé sur le terrain (test préalable).

350. Le questionnaire devrait être le plus simple et le plus bref possible, avoir une structure logique et donner des définitions et des directives claires. D'une manière générale, plus le questionnaire est long, plus le taux de réponse globale et partielle diminue. Cet effet peut être atténué en accordant une attention particulière à la conception et à la présentation du questionnaire, ainsi qu'en fournissant des notes explicatives et des exemples suffisants et clairs. Il importe surtout de concevoir le questionnaire de manière à ce que les unités qui ne mènent pas de façon systématique des activités d'innovation, y répondent néanmoins.

351. Les répondants pourraient bien améliorer leur compréhension du questionnaire en progressant de question en question. Cela implique que leurs réponses peuvent dépendre de l'ordre des questions. L'adjonction ou la suppression d'une catégorie peut influer sur les réponses.

352. Il convient de vérifier, pour toutes les questions d'un questionnaire, s'il est nécessaire d'y inclure une catégorie "sans objet", qui permette de distinguer cette réponse d'une non-réponse.

353. L'expérience montre que l'empressement à remplir des questionnaires sur l'innovation varie selon les groupes d'unités. Moins les unités s'estiment concernées, comme c'est le cas pour beaucoup d'unités de taille réduite et de secteurs dans lesquels l'innovation est une notion relativement peu familière, moins elles sont disposées à participer aux enquêtes sur l'innovation. Une solution consisterait à élaborer des questionnaires spécifiques pour ces groupes, par exemple en limitant le questionnaire à quelques questions essentielles. 
354. En cas d'enquête internationale sur l'innovation, une attention particulière devrait être portée à la traduction et à la conception du questionnaire. Des différences même mineures entre les questionnaires nationaux peuvent restreindre fortement la comparabilité des résultats. De telles différences peuvent provenir, par exemple, des traductions, de changements dans l'ordre des questions, ou encore de l'adjonction ou de la suppression de catégories. Une bonne traduction, tenant compte des particularités locales (telles que le système juridique d'un pays) contribuera à éviter les malentendus sur les concepts et les définitions. Les problèmes conceptuels ne devraient pas être masqués par des traductions ambiguës.

\subsection{Enquêtes sur l'innovation et sur la R-D}

355. Puisque la R-D et l'innovation sont des phénomènes liés, les pays peuvent songer à combiner les enquêtes sur la R-D et sur l'innovation (voir chapitre 5, section 6.1). Un certain nombre d'arguments militent en faveur et contre un tel lien :

- premièrement, dans une enquête combinée, la charge que représentent globalement les réponses pour les unités enquêtées diminuerait (un questionnaire unique au lieu de deux enquêtes distinctes posant en partie les mêmes questions). Toutefois, la charge de travail au niveau individuel ne sera peut-être pas nécessairement réduite. Avec deux enquêtes, on peut mieux répartir la charge entre les unités. En outre, le fait de combiner les enquêtes peut réduire le taux de réponse, puisque le questionnaire sera plus long que chacun des questionnaires des enquêtes dissociées ;

- deuxièmement, une enquête combinée offre la possibilité d'analyser les relations entre les activités de R-D et d'innovation au niveau de l'unité. Cette possibilité est restreinte dans le cas d'enquêtes distinctes, notamment lorsqu'elles sont exécutées par des organismes différents ;

- troisièmement, les unités qui ne sont pas très familiarisées avec les concepts de R-D et d'innovation peuvent les confondre dans une enquête combinée. La réalisation d'enquêtes dissociées entraînera probablement moins de confusion à cet égard ;

- quatrièmement, au moins dans les grandes unités, les réponses aux questions sur la R-D et sur l'innovation peuvent venir de personnes différentes, de sorte que l'enquête combinée risque de ne pas être avantageuse ;

- enfin, les cadres des deux enquêtes sont différents. Les combiner impliquerait l'envoi de questions sur la R-D à un grand nombre d'unités n'exécutant pas de R-D ${ }^{36}$ qui sont incluses dans la population cadre de l'enquête sur l'innovation, et cela ferait augmenter le coût de l'enquête combinée.

356. En résumé, on peut avancer des arguments en faveur et à l'encontre du fait de combiner les enquêtes sur la R-D et celles sur l'innovation, de sorte que l'on peut difficilement formuler une recommandation claire. Il appartient à chaque pays menant ces deux types d'enquêtes de décider luimême si ce sont les avantages ou les inconvénients qui l'emportent, en tenant compte des caractéristiques particulières de leurs systèmes nationaux.

\section{EXÉCUTION DES ENQUÊTES PAR SONDAGE}

357. Dans la plupart des cas, les enquêtes sur l'innovation sont des enquêtes par sondage portant sur des échantillons aléatoires. Les publications spécialisées proposent des techniques d'échantillonnage fort diverses, comme la technique d'échantillonnage aléatoire simple, les techniques de stratification ou les 
techniques d'échantillonnage en grappes. Dans le passé, on a constaté que les enquêtes fondées sur des échantillons stratifiés donnaient des résultats fiables.

358. Si l'on a recours à des techniques de stratification, il convient de respecter certaines règles générales en ce qui concerne la sélection des critères de stratification. En principe, la stratification de la population devrait conduire à des strates aussi homogènes que possible eu égard au phénomène considéré, autrement dit, les strates d'unités utilisées pour les enquêtes sur l'innovation devraient être constituées d'unités aussi semblables que possibles, du moins en ce qui concerne leurs activités d'innovation ou hors innovation. Il est aujourd'hui notoire que les activités d'innovation d'unités appartenant à des industries et à des catégories de tailles différentes diffèrent sensiblement en général. Il est donc recommandé que la stratification de l'échantillon aléatoire utilisé pour une enquête sur l'innovation se fonde sur la taille et l'activité principale des unités.

359. La taille des unités devrait être mesurée par les effectifs de personnel. Compte tenu des différents types d'unités (voir chapitre 4) et des différentes conventions nationales, il est assez difficile de formuler des recommandations générales sur les catégories de tailles. On trouvera ci-après quelques recommandations énoncées à des fins analytiques, qui pourraient aussi s'appliquer à la stratification.

360. La stratification des unités en fonction de leur activité principale devrait se fonder sur les classifications CITI Rev. $3^{37} /$ NACE Rev. $1^{38}$. Là encore, aucune recommandation générale ne peut être formulée quant au niveau des classifications sur lesquelles la stratification devrait se fonder. La décision dépend éminemment de la situation nationale. Prenons le cas d'une économie spécialisée dans la production de bois (Division 20 de la CITI Rév. 3 / NACE Rév. 1) ; pour ce pays, une autre subdivision au niveau du groupe, voire de la classe, pourrait être utile, contrairement à une autre économie où la production de bois a peu d'importance. Cependant l'agrégation des unités ne devrait pas aller au-delà de la division (indicatif à deux chiffres de la CITI Rév. 3 et de la NACE Rév. 1).

361. Si les aspects régionaux sont importants, comme c'est le cas pour les pays de l'Union européenne, la stratification devrait également inclure la dimension régionale. Il convient d'utiliser une classification régionale appropriée (NUTS ${ }^{39}$ pour l'Union européenne). La stratification dans le cas des États membres de l'UE devrait se situer au moins au niveau des indicatifs à un chiffre de la NUTS.

362. Afin de garantir une grande précision, le taux de sondage ne devrait pas être identique pour toutes les strates. Il est généralement recommandé que le taux de sondage d'une strate soit d'autant plus élevé que le nombre de ses unités dans la population objet de l'enquête est plus petit, et que la population de la strate est plus hétérogène. Les taux de sondage devraient atteindre jusqu'à 100 pour cent, par exemple dans les strates comptant peu d'unités, comme cela pourrait être le cas des strates comportant de grandes unités dans certaines industries (ou certaines régions). Un autre facteur à prendre en considération lors de la fixation des divers taux de sondage est la propension à répondre observée dans les strates. Des exemples de strates dans lesquelles la propension à répondre risque d'être relativement faible sont celles constituées de petites unités, qui ne sont peut-être pas très familiarisées avec le concept d'innovation.

363. Pour obtenir les informations relatives à la population objet de l'enquête, il faut extrapoler les résultats des enquêtes par sondage. Il existe diverses méthodes d'extrapolation des résultats obtenus par sondage. La plus simple est la technique de l'extrapolation libre, dans laquelle les différents résultats sont pondérés par l'inverse des taux de sondage appliqués aux unités sondées (facteurs d'extrapolation). Si l'enquête repose sur un échantillon stratifié, la technique d'extrapolation libre devrait être appliqué séparément à chacune des strates, surtout lorsque les taux de sondage sont différents selon les strates. Les facteurs d'extrapolation peuvent être modifiés dans le cas où le taux de non-réponse pour l'unité dépasse un certain seuil. 
364. On est amené à appliquer des techniques d'extrapolation à des variables tant quantitatives que qualitatives, mais de manières différentes. Dans le cas des variables quantitatives, les valeurs observées peuvent être pondérées directement, alors que dans le cas des variables qualitatives, il faut calculer les fréquences.

\section{ESTIMATION DES RÉSULTATS - LE PROBLÈME DE LA NON-RÉPONSE}

365. Dans la pratique, les réponses aux enquêtes sur l'innovation sont toujours incomplètes, quelle que soit la méthode d'enquête utilisée. On peut distinguer deux types de valeurs manquantes : non-réponse globale ou non-réponse partielle. La non-réponse globale signifie qu'une unité déclarante ne répond pas du tout. Les raisons en peuvent être, par exemple, que l'organisme de sondage ne réussit pas à contacter l'unité déclarante, ou que l'unité déclarante refuse de répondre. En revanche, la non-réponse partielle correspond au cas où une unité répond d'une façon générale, mais laisse au moins une question sans réponse. On peut considérer comme relevant de la non-réponse partielle même le cas extrême où toutes les questions sauf une restent sans réponse.

366. Les non-réponses globales ou partielles seraient moins problématiques si les valeurs manquantes se répartissaient de façon aléatoire sur toutes les unités sondées et toutes les questions. Dans la réalité, cependant, les deux types de valeurs manquantes sont biaisés par rapport à certaines caractéristiques de la population et du questionnaire. L'expérience de l'Enquête communautaire sur l'innovation montre que les non-réponses globales se concentrent, par exemple, dans certaines situations ("nous sommes confrontés à des problèmes économiques sérieux et nous n'avons pas le temps de remplir votre questionnaire") ou dans certaines industries ("l'innovation est un concept inconnu dans notre branche"). Les non-réponses partielles se produisent plus souvent lorsque la question est (ou semble être) plus difficile. Un exemple frappant de non-réponse à une question dans l'Enquête communautaire sur l'innovation a été relevé dans le cas de la question sur les dépenses d'innovation.

367. Il est évident que les non-réponses globales ou partielles affectent la comparabilité des résultats des enquêtes nationales et internationales sur l'innovation. Il y a lieu d'élaborer et d'appliquer des méthodes appropriées afin de surmonter ce problème. Etant donné que des méthodes différentes peuvent aboutir à des résultats différents, il convient de se conformer à certaines recommandations générales, faute de quoi les différences relevées dans le temps et/ou entre les pays dans les résultats des enquêtes sur l'innovation peuvent être provoquées par l'utilisation de démarches différentes pour réduire le biais induit par les non-réponses globales et partielles.

368. Pour des raisons aussi bien pratiques que théoriques, une solution recommandée pour surmonter le problème de la non-réponse partielle est un groupe de méthodes appelées "méthodes d'imputation". Fondamentalement, les méthodes d'imputation cherchent à estimer des valeurs manquantes sur la base d'informations supplémentaires. Ces informations peuvent provenir de la même enquête, d'enquêtes antérieures ou de certaines autres sources connexes. Une catégorie spéciale de techniques d'imputation, à savoir les méthodes dites de "hotdecking" (bouche-trou), a été utilisée dans le processus de nettoyage des résultats nationaux de l'Enquête communautaire sur l'innovation. Il s'agit, en l'occurrence, d'estimer les valeurs manquantes sur la base des informations disponibles dans la même enquête. Ces méthodes ellesmêmes sont d'une grande diversité, consistant par exemple à remplacer les valeurs manquantes pour chaque variable par la moyenne de la strate, et à utiliser des techniques de régression ou des techniques d'approximation, où les valeurs manquantes sont remplacées par les valeurs obtenues pour l'unité qui est la plus semblable eu égard aux autres variables (pertinentes). La décision relative à la méthode de "hodecking" la mieux appropriée devrait également se fonder sur le type de variable (variables quantitatives par opposition à des variables qualitatives). 
369. Le choix de la méthode à utiliser pour surmonter le problème de la non-réponse globale dépendra du niveau de non-réponse. Si le taux de non-réponse est assez faible ${ }^{40}$, les facteurs d'extrapolation devraient être corrigés directement. Dans le cas de l'extrapolation libre, les facteurs ne devraient pas être calculés sur la base des unités sélectionnées pour l'enquête, mais sur la base des unités qui ont répondu. Cette procédure repose sur l'hypothèse que le comportement novateur des unités, qu'elles aient ou non répondu, est identique. Cette hypothèse pourrait être vérifiée par une analyse des non-réponses. Même si cette hypothèse est erronée, le biais ainsi introduit peut être négligé tant que la fraction de non-réponses globales est assez faible.

370. En revanche, si le taux de non-réponse globale est très élevé, aucune méthode ne peut être recommandée pour résoudre le problème. Dans un pareil cas, les résultats de l'enquête sur l'innovation ne peuvent être utilisés qu'à des fins descriptives. On ne devrait en tirer aucune conclusion supplémentaire, même sur la population cible en général, car le biais sera trop élevé.

371. Dans tous les autres cas, autrement dit lorsque le taux de non-réponse globale se situe au-dessus d'un seuil plancher, mais en dessous d'un seuil plafond, certaines techniques plus complexes, et, pour une part, plus coûteuses, sont recommandées. Une solution consisterait à sélectionner au hasard les unités déclarantes qui ont répondu jusqu'à ce que le taux de réponse soit de 100 pour cent, c'est-à-dire à utiliser les résultats des unités sélectionnées aléatoirement deux fois, voire davantage. D'autres méthodes sont fondées sur les résultats de l'analyse des non-réponses. L'analyse des non-réponses a pour objectif d'obtenir des informations sur la raison pour laquelle les unités déclarantes n'ont pas répondu. Dans cette enquête sur les non-réponses, il convient de contacter par téléphone ou par courrier (à l'aide d'un questionnaire très simple ne dépassant pas une page) les unités qui n'ont pas répondu et de leur demander certaines informations générales, comme leur code CITI / NACE ou leur taille, si ces données ne sont pas déjà disponibles auprès d'autres sources, telles que les registres des entreprises, et la raison pour laquelle elles n'ont pas répondu, et de leur poser un nombre limité de questions clés de l'enquête originale afin de déterminer si les résultats sont biaisés. Ces informations peuvent alors être utilisées pour corriger les facteurs d'extrapolation ${ }^{41}$. Les résultats de l'analyse des non-réponses ne devraient être utilisés que si le taux de réponse à l'enquête sur les non-réponses dépasse 80 pour cent.

372. Les résultats de l'analyse des non-réponses peuvent également être utilisés pour corriger les valeurs des indicateurs d'innovation tels que la proportion d'unités innovantes ${ }^{42}$.

\section{PRÉSENTATION DES RÉSULTATS}

373. Les résultats des enquêtes sur l'innovation peuvent être utilisés à des fins descriptives ou à des fins inductives. L'objectif de l'analyse descriptive est de décrire les unités statistiques du point de vue de leurs activités novatrices ou non novatrices, sans tirer de conclusions sur l'enquête qui la sous-tend ou sur la population cible (s'il ne s'agit pas d'un recensement). Dans ce type d'analyse, les résultats sont considérés sans autre pondération, tels qu'ils ont été observés pour les différentes unités. Aucune généralisation des résultats au niveau de l'enquête ou de la population cible n'est possible, car le chiffre ne fait référence qu'aux unités ayant répondu. Pour cette sorte d'analyse, le taux de non-réponse globale est sans importance.

374. Au contraire, l'analyse inductive a pour objectif de tirer des conclusions sur la population objet de l'enquête, autrement dit les résultats devraient donner une estimation (représentative) de la situation de l'ensemble des unités statistiques tant observées que non observées. L'analyse inductive nécessite une pondération des résultats. Pour ce type d'analyse, le taux de non-réponse globale revêt une grande 
importance : si le taux de non-réponse globale dépasse un certain seuil, l'analyse inductive est dénuée de sens.

375. Comme il est mentionné plus haut, la plupart des enquêtes sur l'innovation reposent sur un échantillon aléatoire. Les résultats de ces enquêtes comprendront deux types d'erreurs: les erreurs aléatoires dues aux processus aléatoires utilisés pour la sélection des unités, et les erreurs systématiques regroupant toutes les erreurs non aléatoires (biais). Pour avoir au moins une idée du niveau d'erreur, il est recommandé de calculer non seulement les valeurs (moyennes) pour les indicateurs pertinents, tels que la proportion d'unités innovantes ou les dépenses moyennes d'innovation par unité innovante, mais aussi leurs écarts-types et/ou intervalles de confiance. Dans l'hypothèse d'une absence de biais, la probabilité est très élevée pour que la totalité des valeurs vraies mais inconnues dans la population objet de l'enquête soit incluse dans l'intervalle de confiance. L'écart-type donne la limite inférieure de l'erreur totale inconnue entachant les indicateurs considérés.

376. Pour améliorer la comparabilité des résultats des enquêtes sur l'innovation au niveau international, la définition d'un ensemble de tableaux fondamentaux est préconisée. Ces tableaux devraient être dérivés des tableaux nationaux, qui peuvent être beaucoup plus détaillés.

\section{FRÉQUENCE DES COLLECTES DE DONNÉES}

377. Des considérations théoriques et pratiques, de même que les besoins des utilisateurs au niveau international, national et régional, déterminent la fréquence des enquêtes sur l'innovation. Etant donné l'importance croissante de l'innovation pour la croissance des économies ${ }^{43}$, on a besoin de données plus régulières et plus à jour. De ce point de vue, les informations sur les activités d'innovation devraient en principe être collectées en continu, autrement dit des enquêtes sur l'innovation devraient être effectuées chaque année. Ce point de vue est conforté par des considérations théoriques laissant supposer que les activités d'innovation viennent par vagues, ce qui rend les résultats des enquêtes non annuelles très tributaires de l'époque à laquelle il est procédé à l'enquête. Cependant, seuls quelques pays peuvent se permettre de mener tous les ans des enquêtes sur l'innovation.

378. On s'accorde de façon générale à penser, au niveau international, que les enquêtes sur l'innovation devraient être menées le plus souvent tous les deux ans, voire plus rarement encore pour certaines variables. Si cette démarche est adoptée, l'analyse des séries chronologiques sera également possible, du moins à long terme. Quant à savoir si la fréquence devrait être de deux ans ou davantage, la réponse dépend de différents facteurs, tels que la périodicité des enquêtes sur la R-D, ou les exigences légales éventuelles à l'échelon national ou européen.

379. En plus de ces enquêtes générales sur l'innovation, il est recommandé de procéder à des études plus détaillées de certaines sous-populations ou de certains sujets spécifiques. 


\section{ANNEXE 1 \\ UTILISATION DE L'APPROCHE OBJET POUR LA COLLECTE DE DONNÉES SUR L'INNOVATION}

\section{INTRODUCTION}

380. Le chapitre de ce manuel décrit deux manières de recueillir des données sur l'innovation. La collecte d'informations sur les activités d'innovation des firmes (innovantes et autres) connue comme l'"approche sujet" et la collecte d'informations sur des innovations particulières, l"approche objet". Ces expressions seront utilisées dans la suite de l'annexe 1.

381. Le manuel recommande aux pays d'adopter l'approche sujet pour mener leurs enquêtes sur l'innovation. Pourtant, l'approche objet peut fournir des informations supplémentaires intéressantes surtout si elle est utilisée conjointement avec l'approche sujet. On décrira donc dans cette annexe la façon dont les pays peuvent employer l'approche objet en même temps que l'approche sujet pour effectuer des enquêtes sur l'innovation. Mais on peut également recueillir des données sur des innovations particulières par des méthodes reposant sur le dépouillement de la presse spécialisée. Ces méthodes seront examinées dans la deuxième partie de cette annexe.

\section{ENQUETES SUR DES INNOVATIONS PARTICULIERES}

\subsection{Questions abordées lors de l'utilisation de données fournies par l'approche objet}

382. Dans certains cas, les pouvoirs publics chercheront par leurs politiques d'innovation à promouvoir un type particulier d'innovations. Il leur faudra donc des données sur des innovations spécifiques. Dans d'autre cas, les programmes d'innovation viseront des entreprises, et tendront, par exemple, à encourager certains types de comportement innovateur. Il s'agira alors de recueillir des données propres à l'entreprise qui permettent de surveiller la mise en oeuvre du programme. Par conséquent, les pouvoirs publics auront besoin de données tant sur les firmes innovantes que sur les innovations qu'elles accomplissent. D'autre part, on sait par expérience qu'il est plus facile de recueillir certaines données sur une innovation particulière que sur la firme innovante considérée.

383. Pour les organismes qui collectent ces données, une des difficultés consiste à mesurer les dépenses d'innovation et à établir le lien entre ces dépenses et l'impact financier de l'innovation. En effet, même pour une innovation particulière, les dépenses peuvent porter sur plusieurs années, et risquent de chevaucher plusieurs périodes de référence dans les enquêtes. De même, les bénéfices tirés de ces innovations peuvent également être ressentis sur diverses périodes ne coïncidant pas forcément avec les exercices sur lesquels les dépenses sont supportées. La difficulté ne fait qu'augmenter quand les firmes réalisent plusieurs innovations au cours d'une même période d'observation. Il devient alors extrêmement difficile de mesurer, pour l'ensemble de la firme, les dépenses réalisées pour toutes ses innovations puis de 
les rapporter aux répercussions financières. Pour une innovation spécifique, notamment l'innovation principale de la firme, les difficultés sont moindres. Les entreprises sont, en effet, capables d'indiquer les dépenses correspondant à leur innovation principale, ainsi que les bénéfices qu'elles en ont retirés.

384. Il est également difficile de bien mesurer par l'approche sujet le cycle de vie nécessaire à l'accomplissement des innovations. De nombreuses firmes réalisent en général, plusieurs innovations pendant une période donnée de sorte que ces données agrégées ne veulent rien dire lorsque l'analyse est effectuée au niveau de la firme. En revanche, il est assez facile d'étudier le cycle de vie d'une innovation donnée en mesurant le temps nécessaire pour que l'innovation en question parvienne au stade de la commercialisation et le temps nécessaire pour que la firme récupère son investissement. Ce type de données est, pour les analystes des politiques, une source de renseignements très utile.

385. L'approche objet se révèle, en outre, très précieuse lorsqu'on étudie la nouveauté de l'innovation. Dans un but politique, il est, en effet, très important de distinguer entre les caractéristiques des innovations qui constituent une nouveauté pour le monde, voire pour un pays, de celles qui ne sont une nouveauté que pour l'entreprise. Comme la plupart des firmes introduiront une variété d'innovations sur la période observée, il sera très difficile, par l'approche sujet, de rendre compte de la nouveauté de l'ensemble des activités d'innovation de l'entreprise. De plus, les données sur la nouveauté d'une innovation présentent, pour les pouvoirs publics, un intérêt considérable, car elles font ressortir, par exemple, les secteurs, qu'il s'agisse d'industries spécifiques ou de régions, qui sont à la pointe du progrès et ceux qui ne le sont pas.

386. L'approche objet permet également de préciser s'il s'agit d'une innovation de produit ou de procédé (ou une innovation mixte de produit et de procédé) et d'indiquer le but de l'innovation. De nombreuses firmes réaliseront tant des innovations de produits que des innovations de procédés au cours d'une période d'observation donnée si bien que cette classification peut se révéler difficile à appliquer lors de l'analyse des données recueillies par l'approche sujet. En revanche, les données fournies par l'approche objet sur des innovations spécifiques sont plus faciles à classer en innovations de produits, innovations de procédés, ou innovations mixtes.

387. Actuellement, les données sur les objectifs de l'innovation, les domaines bénéficiant de l'innovation et les sources d'informations ou les idées à l'origine de l'innovation sont recueillies auprès de l'entreprise par l'approche sujet. Toutefois, il pourrait être plus facile d'attribuer ces données à une innovation particulière qu'à l'ensemble des activités d'innovation de la firme. Pour ce type de données, l'approche objet devrait fournir des renseignements plus significatifs et plus précis.

\subsection{Inconvénients de l'approche objet}

388. On peut recourir à l'approche objet pour recueillir des données sur chacune des innovations d'une firme. Cependant, cette démarche risque de représenter pour l'entreprise une charge de travail excessive étant donné qu'elle ne sera pas en mesure de fournir les informations détaillées demandées dans les formulaires si elle ne consigne pas en permanence les données sur toutes ses innovations. Par conséquent, il n'est pas recommandé d'utiliser l'approche objet de cette façon.

389. Sachant que l'intérêt des responsables des politiques, en matière d'innovations spécifiques, se porte surtout sur les innovations majeures, de grande portée, il est possible de restreindre le champ d'investigation à ces innovations principales. Il s'agira alors de recueillir des données sur l'innovation la plus importante, voire sur plusieurs innovations importantes. De toute évidence, le choix de plusieurs innovations élargira la base de données analysables. Mais il faudra alors tenir compte de la charge de 
travail que les entreprises pourront supporter si l'on augmente le volume d'informations demandées. En se contentant de demander aux entreprises des informations sur leur innovation principale, les responsables politiques devraient disposer d'un volume suffisant d'informations à analyser.

390. Il faut souligner que cette démarche interdit de considérer les statistiques ainsi recueillies comme représentatives de la totalité des innovations réalisées par un pays au cours d'une période donnée. Ces statistiques ne représenteront qu'un sous-ensemble des innovations produites, et les analystes devront se garder d'en tirer des conclusions sur l'ensemble des innovations. De ces données ils pourront en revanche déduire des conclusions relatives aux innovations importantes, notamment s'ils classent ces innovations en fonction d'autres paramètres comme les dépenses d'innovation, la taille de l'entreprise, etc.

\subsection{Mise en oeuvre de l'approche objet}

391. Conformément à la description figurant dans le corps de ce manuel, l'approche objet est conçue comme un complément de l'approche sujet et ne saurait s'y substituer.

392. L'approche objet sera utilisée de préférence en complément de l'approche sujet pour la compilation de données sur l'innovation. Employée ainsi, elle ne nécessite pas d'enquête supplémentaire. Il s'agit simplement d'incorporer quelques questions supplémentaires sur l'innovation la plus importante d'une firme. De cette manière, les données recueillies par l'approche objet peuvent être rapportées à des données sur la firme, comme ses finances et sa production au niveau de l'unité sur laquelle porte l'enquête, de façon à identifier des corrélations éventuelles avec l'innovation principale.

393. Il vaut mieux laisser l'entreprise définir elle-même quelle est son innovation la plus importante. Les résultats d'une enquête effectuée en 1994 par l'Australian Bureau of Statistics (ABS) ont montré l'intérêt de cette méthode du point de vue de la collecte, même si les données ainsi recueillies portent sur une gamme d'innovations différentes. Il ne semble pas que ce soit un problème majeur étant donné qu'il est toujours possible de recueillir des statistiques synthétiques en regroupant des innovations importantes similaires en fonction de leurs caractéristiques : type, dépenses d'innovation, cycle de vie, etc.

\subsection{Expérience de la collecte de données par l'approche objet}

394. Le Science Policy Research Unit (SPRU) de l'université du Sussex a utilisé cette approche au cours des années 70 jusqu'au début des années 80. Le SPRU a ainsi consigné les 4000 innovations les plus importantes dans l'industrie manufacturière britannique entre 1945 et 1983 . Après avoir sélectionné les innovations sur lesquelles faire porter son enquête, le SPRU a recueilli des informations sur ces innovations, ainsi que sur les caractéristiques des firmes innovantes au moment où elles ont introduit ces innovations.

395. Le SPRU n'a pas été le seul à utiliser ce type de méthodologie combinée. Les États-Unis avaient procédé à des enquêtes apparentées entre le milieu des années 70 et le début des années 80 . Il a été fait référence à des travaux similaires au Canada, en France et en Allemagne, à peu près à la même époque.

396. Plus récemment, l'ABS en Australie et Statistique Canada ont recueilli, à l'aide du même formulaire ou dans le cadre d'enquêtes mixtes, des informations sur les activités d'innovation des entreprises (par l'approche sujet), ainsi que certaines informations sur leurs principales innovations (par l'approche objet). Cette alliance de l'approche sujet et de l'approche objet a été un succès. 


\subsection{Données susceptibles d'être recueillies par l'approche objet}

397. On recueillera par l'approche objet des données différentes de celles recueillies par l'approche sujet pour la bonne raison que l'objet mesuré diffère : l'innovation principale au lieu de la firme innovante.

398. Trois grandes catégories de données peuvent être recueillies par l'approche objet : des informations descriptives, des informations quantitatives et des informations qualitatives. Les données figurant ci-dessous sont celles que l'on recommande aux organismes d'enquête de recueillir sur l'innovation la plus importante commercialisée par la firme pendant la période d'observation.

\subsubsection{Données descriptives}

\subsubsection{Description de l'innovation principale}

399. Fournit des données descriptives sommaires sur le processus d'innovation spécifique.

\subsubsection{Classification par type d'innovation}

400. Fournit des détails sur le type d'innovation : l'innovation décrite est-elle une innovation de produit ou de procédé, un nouveau produit, un produit modifié ou la combinaison de plusieurs types d'innovation?

\subsubsection{Nouveauté de l'innovation}

401. Fournit des détails sur le degré de nouveauté de l'innovation. La nouveauté d'une innovation peut être définie au moyen d'un certain nombre de variables techniques, ou en fonction du marché.

a) Classification par type de nouveauté au moyen de variables techniques

402. Les informations peuvent être obtenues en demandant à l'entreprise de cocher les cases correspondant aux catégories appropriées.

- Innovations de produits

- utilisation de nouveaux matériaux ;

- utilisation de nouveaux produits intermédiaires ;

- nouveaux éléments fonctionnels ;

- utilisation de technologie radicalement nouvelle ;

- fonctions fondamentalement nouvelles (produits radicalement nouveaux).

- Innovations de procédés

- nouvelles techniques de production;

- changement dans l'organisation en rapport avec l'introduction de nouvelles technologies ;

- nouveaux logiciels professionnels. 
b) Classification par type de nouveauté du point de vue du marché

403. Classification par type de nouveauté

- nouveauté pour l'entreprise seulement;

- nouveauté pour l'industrie du pays considéré ou pour le marché sur lequel l'entreprise opère ;

- nouveauté pour le monde entier.

\subsubsection{Nature de l'innovation}

404. Cette classification pourrait fournir des renseignements supplémentaires utiles, car elle donne une indication de la source de l'innovation.

Classification selon la nature de l'innovation :

- application d'une percée scientifique ;

- innovation technique notable ;

- amélioration ou modification technique ;

- transfert d'une technique à un autre secteur ;

- adaptation d'un produit existant à un nouveau marché.

\subsubsection{Données quantitatives}

\subsubsection{Dépenses d'innovation}

405. Ces données fournissent des renseignements détaillés sur les dépenses correspondant à l'innovation principale, que l'on peut ensuite relier aux dépenses d'innovation totales de la firme afin d'en déduire le contexte dans lequel a eu lieu l'innovation décrite.

\subsubsection{Impact de l'innovation}

406. Etant donné les problèmes que pose la collecte d'informations relatives à l'impact de l'innovation sur la firme, il sera peut-être utile de limiter le recueil d'informations détaillées à l'innovation la plus importante, ce qui permettra de procéder à une analyse coûts-avantages plus précise et plus détaillée.

407. On propose d'inclure une question relative à la part des ventes et exportations revenant à la principale innovation technologique de produit qui a été mise sur le marché au cours des trois dernières années ; ou une question relative aux répercussions de la principale innovation technologique de procédé sur l'utilisation des facteurs de production, c'est-à-dire sur l'utilisation de la main-d'oeuvre, la consommation de matières premières, d'énergie et l'utilisation de capital fixe. 


\subsubsection{Cycle de vie de l'innovation}

408. On peut obtenir des renseignements détaillés sur des projets spécifiques, comme le temps écoulé avant d'atteindre la phase de commercialisation ou la période prévue pour récupérer les coûts ou le temps de remboursement.

409. Comme il s'agit essentiellement d'obtenir des données comparatives sur les innovations, non pas de tirer des conclusions sur la totalité des projets d'innovations, les informations numériques recherchées à travers les questions ne doivent pas forcément être très précises. Cette partie du formulaire d'enquête peut donc être simplifiée en proposant des fourchettes de valeurs à cocher.

\subsubsection{Données qualitatives}

\subsubsection{Bénéfices retirés de l'innovation}

410. On peut recueillir sur les bénéfices retirés d'innovations spécifiques, des informations du même type que celles recueillies par l'approche sujet.

\subsubsection{Sources d'informations ou d'idées pour l'innovation}

411. On peut recueillir sur des innovations spécifiques des données du même type que celles recueillies par l'approche sujet.

412. Bien que certaines des données recueillies par l'approche objet soient semblables à celles que l'on obtiendrait par l'approche sujet, l'accent n'est pas mis du tout sur les mêmes aspects; il en va de même pour les réponses des entreprises. Par conséquent, les utilisations des données diffèrent aussi et peuvent se compléter sans risque véritable de chevauchement.

\subsubsection{Diffusion de l'innovation}

413. Au chapitre 1, la diffusion est définie comme étant la manière dont les innovations se répandent, par l'intermédiaire des mécanismes du marché ou autrement. Sans diffusion, une innovation n'aura pas d'incidences économiques. Quelques indicateurs de diffusion sont présentés ci-après.

a) Secteurs utilisateurs

414. Théoriquement, les innovations pourraient être classées selon trois critères :

- le secteur d'activité du producteur ;

- le groupe technologique (ou groupe de produits) auquel appartient l'innovation ;

- le secteur d'utilisation.

415. Le premier critère est abordé dans la section sur les classifications.

416. A ce jour, seules quelques enquêtes sur l'innovation comportaient une question relative au secteur utilisateur. Pour l'innovation la plus importante, les entreprises étaient priées d'indiquer le domaine d'utilisation type. On propose de poser des questions sur le groupe de produits et le secteur d'utilisation. 
417. Contrairement à ce qui se passe avec l'approche sujet, la période de référence applicable lorsqu'on utilise l'approche objet est la durée de vie du projet d'innovation et non les différentes périodes de référence utilisées dans le reste de ce manuel. Les différences entre périodes de référence devront être prises en compte chaque fois que l'on reliera des données recueillies par l'approche sujet à celles obtenues par l'approche objet.

\section{INDICATEURS D'OUTPUTS DE L'INNOVATION FONDÉS SUR LA PRESSE SPÉCIALISÉE}

418. La première partie de cette annexe décrit l'utilisation de l'approche objet dans le cadre d'enquêtes sur l'innovation. La deuxième partie sera consacrée à la collecte d'informations sur des innovations particulières décrites dans des revues techniques ou professionnelles. Cette méthode est souvent appelée "méthode d'analyse des outputs de l'innovation fondée sur l'analyse de la presse spécialisée". Bien qu'elle ne fasse pas appel aux techniques statistiques classiques utilisées pour les enquêtes sur l'innovation (population, échantillon, etc.) et qu'elle se limite à des données sur les innovations de produits, cette méthode a le mérite de ne pas exiger des entreprises un lourd effort d'information. Elle a été appliquée ces dernières années en Italie (Santarelli et Piergiovanni, 1996), au Royaume-Uni (Combs et al. 1996), aux États-Unis, en Autriche, en Irlande et aux Pays-Bas (voir les contributions diverses dans l'ouvrage publié sous la rédaction de Kleinknecht et Bain, 1993).

\subsection{Méthodologie}

419. Les firmes sont soucieuses de faire connaitre au public les nouveaux produits et services qu'elles introduisent sur un marché. Les communiqués de presse à l'adresse des revues professionnelles et techniques sont un moyen de communication privilégié. Il existe quelques exceptions à cette règle (par exemple, des produits destinés à des créneaux très limités sur les marchés), mais, en général, on peut supposer que la grande majorité des nouveaux produits et services bénéficient d'une publicité. La totalité des nouveaux produits et services cités dans les articles de ces revues (qui sont souvent regroupés dans une rubrique à part intitulée "Nouveaux produits") devrait dont être retenue lors du dépouillement. Pour éviter de prendre en compte un grand nombre d'innovations mineures ou de contrefaçons, il est recommandé de laisser de côté les publicités. En d'autres termes, seules les innovations que les rédacteurs des revues ont jugées dignes de mentionner seront consignées.

420. Les revues en question comportent généralement une courte description du nouveau produit ou service ainsi que l'adresse et le numéro de téléphone de l'organisation à laquelle s'adresser pour obtenir des informations supplémentaires sur le produit. Il est possible de se constituer ainsi un dossier complet des innovations à condition toutefois de dépouiller une série représentative de revues portant sur les secteurs intéressants. Il est assez difficile d'édicter une règle pour le choix de ces revues. La démarche en trois étapes décrite ci-dessous devrait néanmoins permettre une bonne couverture des secteurs :

- s'appliquer à recenser l'éventail le plus vaste possible de revues spécialisées pertinentes par des recherches dans des bibliothèques spécialisées, et s'efforcer d'en obtenir quelques exemplaires ;

- entrer en relation avec les associations professionnelles de tous les secteurs auxquels on s'intéresse pour les interroger sur les revues qu'elles publient et leur demander si ces revues traitent des nouveaux produits ; 
- téléphoner aux services des relations publiques de firmes appartenant aux secteurs auxquels on s'intéresse pour leur demander à quelles revues ils ont l'habitude d'envoyer des communiqués de presse concernant leurs innovations.

421. En général, on s'efforcera de dépouiller une revue au moins par branche d'activité importante. Le choix entre deux ou plusieurs revues propres à une de ces branches doit toujours s'appuyer sur l'étude d'exemplaires de ces revues. On pourra aussi interroger par téléphone les firmes et organisations professionnelles sur les revues qu'elles jugent les plus pertinentes.

422. Pour la collecte des données, les points suivants devront être considérés :

- les données doivent être recueillies en temps réel, par exemple en s'abonnant aux revues pertinentes de façon à pouvoir contacter les entreprises dès l'annonce d'un nouveau produit. L'expérience nous a appris que, même quelques mois après cette annonce, beaucoup de firmes sont difficiles à retrouver, parce qu'elles ont fait faillite, déménagé, été rachetées, etc. La collecte immédiate d'informations présente d'autres avantages. Lorsque ces informations sont publiées dans une revue, l'entreprise à l'origine de l'innovation s'attend à recevoir des coups de téléphone de clients potentiels et est donc disposée à donner des informations complémentaires. Le moment se prête bien à un entretien téléphonique, et l'on peut espérer être mis directement en relation avec l'interlocuteur le plus compétent ;

- l'entretien téléphonique qui a lieu juste après l'annonce de l'innovation permettra de recueillir divers types d'informations suivant les centres d'intérêt et les ressources dont on dispose. Les questions peuvent porter sur les goulots d'étranglement dans le processus d'innovation, les objectifs de l'innovation, les sources d'informations jugées importantes pour la réalisation de l'innovation, les brevets et/ou licences et autres moyens d'appropriation des bénéfices de l'innovation, les réseaux de R-D et modes d'acquisition de technologie, le rôle des infrastructures publiques de R-D ou la participation de l'entreprise à des programmes publics d'innovation, etc. (pour des détails voir Keinknecht et Bain, 1993, pp. 195-198). La méthode des indicateurs des outputs de l'innovation fondés sur la presse spécialisée a le mérite, non négligeable, de permettre de poser des questions concernant le projet, tandis que les enquêtes sur la R-D et celles sur l'innovation font porter ces questions sur la firme, obligeant les grandes entreprises à fournir des réponses "moyennes" pour un ensemble de projets. De plus, elle autorise la collecte de certains types informations sur toutes les innovations ou au contraire sur un type particulier d'innovation. Le choix des questions peut varier, mais l'on devra toujours poser les suivantes :

- l'adresse complète de l'entreprise ;

- la taille de l'entreprise (selon le nombre d'employés et/ou le volume des ventes) ;

- l'entreprise a-t-elle mis au point l'innovation elle-même ?

- la principale branche d'activité de l'entreprise ;

- le secteur auquel l'entreprise espère vendre son produit ou ses services.

Les deux derniers types d'informations permettront de tracer les flux intersectoriels de technologies entre les "producteurs" et utilisateurs d'innovations ; 
- dans les études récentes effectuées par cette méthode, on s'est servi des paramètres suivants pour classer tous les produits ou services nouveaux ou modifiés :

- le degré de complexité

- le type de produit ou service nouveau ou modifié ;

- les propriétés du produit ou service nouveau ou modifié ;

- l'origine du produit nouveau ou modifié.

423. On y distingue trois niveaux de complexité :

- élevé : l'innovation est un système comportant de multiples pièces ou composants relevant souvent de différentes disciplines (par exemple, un satellite d'observation météorologique ou un avion)

- moyen : l'innovation comporte un petit nombre de pièces ou de composants (une imprimante laser, une machine pour l'industrie textile, par exemple);

- faible : innovation simple (par exemple, un frein de bicyclette amélioré).

424. On a répertorié cinq types de modifications :

i) un produit ou service entièrement nouveau ou complètement transformé (disque compact ou service bancaire électronique, par exemple);

ii) un produit ou service accessoire nouveau ou amélioré (siège d'enfant plus sûr pour bicyclette ou meilleure assurance vie se rattachant à un prêt hypothécaire) ;

iii) un produit ou service légèrement amélioré (par exemple un meilleur rendement énergétique des machines ou une meilleure protection des cartes de crédit) ;

iv) une différenciation du produit ou service (nouveau parfum pour un savon par exemple);

v) un processus nouveau ou modifié.

425. La deuxième catégorie "produit ou service accessoire" a été introduite pour recouvrir des modifications légères ou de moindre importance. Ces changements représentent souvent une réelle nouveauté et viendraient grossir, s'ils étaient plus importants, la première catégorie des produits et services entièrement nouveaux ou très transformés.

426. Toutes les propriétés (énumérées dans la courte description du journal) qui distinguent les nouveaux produits de ceux qui existent déjà seront consignées. La liste peut se révéler longue, et certaines propriétés peuvent revenir souvent : "plus convivial", "plus sûr et plus fiable", "plus souple", "gain de temps", "plus précis", "durée de vie accrue", "meilleur pour l'environnement", etc. (voir Kleinknecht et Bain, 1993, p. 62). Ces informations peuvent servir à préciser les caractéristiques du produit nouveau ou modifié et à le classer en fonction du type de changement. Pour distinguer entre un "produit légèrement amélioré" (iii) et une "différenciation du produit" (iv), on peut appliquer la règle suivante : si la description figurant dans la revue mentionne au moins une propriété importante, le produit sera classé 
dans les "produits légèrement améliorés" (iii) si aucune propriété n'est mentionnée, le produit sera classé dans la catégorie "différenciation du produit" (iv).

427. En ce qui concerne l'origine de l'innovation, il conviendra de distinguer entre les entreprises qui ont mis au point l'innovation elles-mêmes et celles qui se contentent de vendre l'innovation d'une autre entreprise. Les entreprises d'import/export qui sont uniquement des canaux de distribution d'innovations mises au point à l'étranger sont une exemple typique de cette dernière catégorie.

\subsection{Avantages et inconvénients de cette méthode}

428. Les propriétés statistiques d'une base de données recueillies par la méthode des indicateurs d'outputs de l'innovation fondés sur la presse spécialisée peuvent paraître sujettes à caution dans la mesure où les procédures d'échantillonnage statistique habituelles sont inapplicables. Il est donc impossible de procéder à des comparaisons simples du nombre d'innovations (par employé ou par unité de volume de ventes) entre différents pays. Le nombre d'innovations enregistrées dépendra en effet du nombre de revues dépouillées. Les comparaisons se trouvent de ce fait réduites à des rapports tels que le pourcentage d'innovations qui revient aux petites entreprises, à certaines régions ou à certains secteurs. Il n'est pas nécessaire pour ce type de comparaison d'analyser la totalité des innovations ; l'essentiel est de recueillir des données de telle manière que les divers types d'entreprises aient les mêmes probabilités d'y voir figurer leurs innovations. D'où l'importance du choix des revues spécialisées (voir ci-dessus).

429. Contrairement aux indicateurs traditionnellement utilisés pour la R-D ou les brevets, la méthode des indicateurs d'outputs de l'innovation fondés sur la presse spécialisée fournit une mesure directe de l'innovation. Autre avantage majeur, elle s'applique en principe à tous les secteurs de l'économie, dont les services et même l'agriculture. De plus, elle prend en compte les innovations de très petites entreprises. Ces dernières sont habituellement oubliées dans les enquêtes postales en raison de la taille fixée pour les entreprises étudiées (par exemple, dix employés). Or il est important d'englober les micro-entreprises car l'expérience passée nous a appris qu'elles sont à l'origine d'une forte proportion des innovations annoncées dans les revues spécialisées (Kleinknecht et Bain, 1993, p. 65) et l'étude systématique du comportement des micro-entreprises en matière d'innovation est encore embryonnaire. La méthode a le mérite supplémentaire de faciliter les répartitions par régions car on connaît l'adresse de l'entreprise qui commercialise le nouveau produit. S'il arrive que le produit n'ait pas été mis au point par l'entreprise qui le commercialise, on obtient néanmoins une meilleure indication des schémas d'innovation régionale qu'avec les données recueillies dans le cadre d'enquêtes classiques sur la R-D ou l'innovation. De plus, les flux intersectoriels de technologies entre les "producteurs" et utilisateurs d'innovations sont plus faciles à identifier qu'avec les résultats des enquêtes postales.

430. Les firmes n'ont pas vraiment intérêt à faire de la publicité sur leurs innovations de procédés. Il peut arriver que l'on rencontre par hasard des innovations de procédés, mais la méthode ne permet pas de les prendre correctement en compte. En revanche, elle recouvre les innovations de procédés incorporées à des nouveaux biens d'équipement (et vendues comme telles).

431. On s'efforcera d'éviter les doubles comptages. Nombreuses sont les innovations qui sont décrites dans plusieurs revues. Si les innovations sont identiques, on peut aisément s'apercevoir qu'on les a comptées deux fois et y remédier. Il n'en va pas de même des imitations très fidèles d'innovations antérieures (véritables) par des concurrents. La difficulté tient au fait que ces imitations sont rarement des copies conformes du produit existant. Un imitateur intelligent s'efforcera dans la plupart des cas d'améliorer le produit qu'il copie et de différencier le sien. Or, pour pouvoir détecter ces imitations intelligentes, celui qui recueille les données doit avoir une connaissance presque encyclopédique des 
nouveaux produits des diverses branches de l'industrie. En s'appliquant à noter toutes les innovations mentionnées dans les revues spécialisées, on risque fort de retenir de nombreuses "innovations" comportant une part plus ou moins importante d'imitation (parfois aussi des connaissances supplémentaires). L'utilisation judicieuse de la classification indiquée précédemment devrait permettre de résoudre ce problème des imitations. Par exemple, dans le cas d'imitations simples, il est fort probable que la description de l'innovation ne contiendra aucune référence à la nouveauté de l'innovation, contrairement aux descriptions d'innovations décrites antérieurement dans la revue. On pourra donc classer le produit d'imitation modifié dans la catégorie "différenciation du produit". Si l'imitateur a introduit quelque modification de son invention dont l'effet est d'améliorer le produit (les propriétés qui ont été améliorées figureront alors dans la courte description donnée dans la revue), l'innovation pourra être classée parmi les "améliorations légères".

432. Enfin, il convient de mentionner la possibilité de relier les informations obtenues par cette méthode avec d'autres jeux de données microéconomiques, celles tirées des enquêtes standard sur la R-D et/ou sur l'innovation ou des informations financières publiées sur les entreprises par exemple, démarche qui peut déboucher sur de nouvelles recherches. La comparaison des données néerlandaises obtenues par cette méthode pour l'année 1989 aux données tirées d'une enquête nationale de 1988 a permis aux analystes de calculer des modèles économétriques expliquant la capacité d'innovation d'une firme et de comparer l'indicateur d'outputs de l'innovation fondé sur la presse spécialisée à l'indicateur de la part des ventes revenant aux produits innovants tiré d'enquêtes sur l'innovation. Il apparaît que les données fournies par la méthode fondée sur la presse spécialisée concordent assez bien avec les indicateurs tirés des enquêtes sur l'innovation (Brouwer et Kleinknecht, 1966, pp. 99-124).

$$
\text { *** }
$$

\section{RÉFÉRENCES}

BROUWER, E. et A. KLEINKNECHT (1996): "Determinants of innovation. A micro-econometric analysis of three alternative innovation output indicators", dans: A. Kleinknecht (éd.): Determinants of innovations. The message from new indicators, Macmillan, Londres, pp. 99-124.

COOMBS, R., P. NARANDREN et A. RICHARDS (1996) : "A literature-based innovation output indicator", Research Policy, Vol. 25, pp. 403-413.

KLEINKECHT, A. et J.O.N. REIJNEN (1993) : "Towards literature-based innovation output indicators", dans : Structural Change and Economic Dynamics, Vol. 4, pp. 199-207.

KLEINKECHT, A. et D. BAIN (éds.) : New concepts in innovation output measurement, Macmillan, Londres et St. Martin's Press, New York.

SANTARELLI, E. et R. PIERGIOVANNI (1996) : "Analysing literature-based innovation output indicators: The Italian experience", Research Policy, Vol. 25, pp. 689-712. 


\section{ANNEXE 2 \\ LA COLLECTE DES DONNÉES SUR L'INNOVATION NON TECHNOLOGIQUE}

\section{Introduction}

433. Les chapitres 1 à 3 de ce manuel décrivent l'optique dans laquelle les pays devraient mesurer l'incidence du changement, et notamment du progrès technologique. Ils font aussi ressortir l'importance de l'innovation non technologique (c'est-à-dire de l'innovation dans l'organisation et dans la gestion) pour l'efficacité économique des entreprises.

434. Dans le chapitre 3, on explique que le changement dans l'organisation n'est considéré comme progrès technologique que s'il se traduit par une variation mesurable de l'output de la firme, qu'il s'agisse de la production ou des ventes. Un changement qui ne concerne que l'organisation proprement dite ne sera pas pris en compte dans le changement technologique.

435. Le récent rapport analytique de l'OCDE intitulé Technologie, productivité et création d'emplois (Paris, 1996) examine de nombreux travaux de recherche qui montrent que le progrès technologique et le changement dans l'organisation sont étroitement liés. Le rapport indique clairement que le progrès technologique est à la fois l'un des facteurs qui incite au changement institutionnel et organisationnel et l'une des conséquences de ce changement. C'est pourquoi il convient, parallèlement aux données sur le progrès technologique, de recueillir aussi un certain nombre de données sur l'innovation dans l'organisation, car les gouvernements en auront de plus en plus besoin pour élaborer leurs politiques.

436. Pour toutes ces raisons, il y a lieu, dans cette version révisée du Manuel d'Oslo, de faire quelques premières suggestions en ce qui concerne la collecte de données sur l'innovation non technologique. Cette annexe ébauche à cet égard une proposition dont on espère qu'elle permettra de mesurer l'ampleur de l'innovation non technologique et son importance pour les firmes.

\section{Qu'entend-on par innovation non technologique ?}

437. Sous sa forme la plus simple, l'innovation non technologique englobe toutes les activités d'innovation qui ne relèvent pas de l'innovation technologique, c'est-à-dire qui ne sont pas liées au lancement d'un bien ou d'un service nouveau ou sensiblement modifié du point de vue technologique, ou à l'utilisation d'un procédé technologiquement nouveau ou sensiblement modifié.

438. Les principaux types d'innovation non technologique seront vraisemblablement les innovations purement organisationnelles et administratives, qui ne sont pas prises en compte dans les enquêtes sur l'innovation technologique, si ce n'est lorsqu'elles s'inscrivent dans un projet d'innovation technologique.

439. D'après l'enquête réalisée par l'Australian Bureau of Statistics (ABS) en 1994, les principaux types d'innovation organisationnelle et administrative sont les suivants : 
- adoption de techniques avancées de gestion (par ex., gestion de la qualité totale, service de qualité totale) ;

- modification importante des structures organisationnelles ;

- adoption d'orientations stratégiques entièrement nouvelles ou modification sensible des orientations stratégiques de l'entreprise.

\section{Expérience acquise dans la mesure de l'innovation non technologique}

440. A ce jour, on a tenté d'évaluer l'ampleur de l'innovation non technologique dans très peu d'enquêtes sur l'innovation. Dans celle qu'a menée l'ABS ${ }^{44}$ sur la période 1993-1994, par exemple, on mesurait l'importance de ce type d'innovation dans toutes les branches d'activité de l'économie australienne. Cette étude était largement expérimentale car elle ne pouvait s'appuyer sur aucune norme internationale et on ne tentait pas de mesurer l'incidence de l'innovation non technologique sur l'efficacité de l'entreprise.

\section{Quelles sont les données qu'il convient de recueillir sur l'innovation non technologique ?}

441. Pour être en mesure d'étudier à fond le concept d'innovation non technologique, l'organisme qui effectue l'enquête doit poser un éventail de questions sur les innovations non technologiques particulières qui sont réalisées. L'idéal serait de consacrer une enquête distincte à l'innovation non technologique, mais cela serait impossible à réaliser dans la pratique, compte tenu de l'insuffisance des ressources et du surcroît de travail qui serait ainsi occasionné aux firmes.

442. Etant donné que le but premier des enquêtes sur l'innovation est de mesurer l'incidence économique de l'activité d'innovation, il est proposé de recueillir, à côté de l'information de base sur l'innovation technologique, un ensemble minimum de données sur l'innovation non technologique.

443. Cet ensemble minimum comprendrait les éléments suivants :

- le type d'innovation non technologique ;

- les avantages économiques qui découlent des activités d'innovation non technologique considérées ;

- les dépenses consacrées à ces activités d'innovation non technologique ;

- le but de ces activités d'innovation non technologique ;

- l'origine des idées/informations pour ces activités d'innovation non technologique.

444. Les données ainsi obtenues devraient renseigner les décideurs politiques sur le processus d'innovation non technologique et sur ses liens avec l'innovation technologique, sans alourdir la tâche des entreprises dans le cadre de l'enquête.

445. Ces questions ne mesureront pas l'impact de l'innovation non technologique sur l'efficacité de l'entreprise. Pour cela il faudrait en effet établir un lien entre les données sur l'efficacité de l'entreprise et 
celles qui concernent l'innovation non technologique. Il conviendrait d'inciter les pays Membres à entreprendre ce type de rapprochement et d'analyse.

446. Il est recommandé que les organismes responsables des enquêtes mettent au point des mesures de l'innovation non technologique en vue de les intégrer à leurs enquêtes sur l'innovation technologique au cours des prochaines années. Ils pourront utiliser comme point de départ l'ensemble minimum de données proposé ci-dessus. 


\section{NOTES}

1 Par exemple, selon une enquête récente, 15 pour cent des entreprises australiennes ont procédé à des innovations organisationnelles et 13 pour cent seulement à des innovations TPP.

2 OCDE (1996), La Stratégie de l'OCDE pour l'emploi - Technologie, productivité et création d'emplois, Volume 1, Paris.

3 On trouvera un précieux tour d'horizon des résultats récents sur l'innovation dans P. Stoneman (éd.) (1995) Handbook of the Economics of Innovation and Technological Change et dans M. Dodgson et R. Rothwell (1994) (éds.), The Handbook of Industrial Innovation, Edward Elgar, Aldershot. Un examen complémentaire des autres incidences économiques du progrès technologique figure dans C. Freeman (1994) "The Economics of Technical Change", Cambridge Journal of Economics 18(5), pp. 463-514.

4 Livre vert sur l'innovation, Bulletin de l'Union européenne, Supplément 5/95, Luxembourg, 1996.

5 Schumpeter, J. (1934), The Theory of Economic Development, Harvard University Press, Cambridge, Massachusetts.

6 Voir Nelson, R. et S. Winter (1982), An Evolutionary Theory of Economic Change, Belknap Press of Harvard University Press, Cambridge, Massachusetts.

7 Voir Romer, P. (1986), “Increasing Returns and Long-Run Growth", Journal of Political Economy 94(5), pp. 1002-1037.

8 Voir Lundvall, B-A (1992) (éd.), National Systems of Innovation: Towards a Theory of Innovation and Interactive Learning, Pinter Publishers, Londres et R. Nelson (1993) National Innovation Systems, Oxford UP, Oxford ; et C. Freeman (1995), "National System of Innovation" in 'historical perspective', Cambridge, J. Econ. 19, pp. 5-24.

9 OCDE (1996), La stratégie de l'OCDE pour l'emploi - Technologie, productivité et création d'emplois, Volume 2 , Paris.

10 Cette façon de dresser un plan des questions politiques est tirée d'une méthode antérieurement examinée au Département de l'industrie, la science et la technologie de l'Australie (1996), Australian Business Innovation: A Strategic Analysis -- Measures of Science and Innovation 5, Australian Government Publishing Service, Canberra.

11 On trouvera un examen approfondi de l'importance capitale de ces questions, ainsi que de leurs incidences pour l'action gouvernementale, dans Dodgson M. et J. Bessant (1996), Effective Innovation Policy: A New Approach, International Thomson Business Press, Londres.

12 Klines, J. et N. Rosenberg (1986), op.cit., pp. 289-291.

13 Klines, J. et N. Rosenberg (1986), “An overview of innovation”, dans R. Landau et N. Rosenberg (éds), The Positive Sum Strategy. Harnessing Technology for Economic Growth, National Academy Press, Washington DC, p. 289.

14 Cela concorde avec un résultat tout à fait certain de l'analyse de l'innovation, à savoir que le succès de l'innovation dépend pour une très large part de la mesure dans laquelle la commercialisation est intégrée aux aspects techniques du processus d'innovation. Pour une étude générale, voir l'ouvrage de C. Freeman (1982), The Economics of Industrial Innovation, 2ème édition, Pinter, Londres, chapitre 5 : "Success and Failure in Industrial Innovation". Hansen et al. (1984) insistent sur ce point dans le cas de la collecte des données, et c'est là un des points forts de leurs travaux d'enquête.

15 Rothwell, R. (1994), "Successful Industrial Innovation: Success, Strategy, Trends", dans M. Dodgson et R. Rothwell, op. cit.

16 Ministres de la science et de la technologie des pays de l'OCDE (1995), Communiqué final de la réunion du Comité de la politique scientifique et technologique au niveau ministériel, 26-27 septembre 1995, OCDE, Paris.

17 Parmi les importants travaux consacrés à ce domaine, on peut citer l'étude de F. Scherer (1982), "Inter-industry Technology Flows in the United States”, Research Policy, Vol. 11, № 5, pp. 227-245 ; celle de A. Jaffe (1986), 
“Technological Opportunity and Spillovers From R\&D: Evidence From Firms' Patents, Profits, and Market Value", American Economic Review, Vol. 76, pp. 984-1001 ; et celle de D. Archibugi (1988), “The Inter-industry Distribution of Technological Capabilities. A Case Study of Italian Patenting in the USA", Technovation, Vol. 7, pp. 259-274.

18 Pavitt, K. (1984), op.cit., pp. 353-364 ; Archibugi et al. (1989), op.cit., Section 5.

19 von Hippel, E. (1988), The Sources of Innovation, Oxford University Press, New York and Oxford, chapitres 3-5, est l'une des rares études systématiques consacrées à ce sujet.

20 Les conséquences de cet aspect pour l'action des pouvoirs publics ont été examinées dans le contexte du RoyaumeUni par K. Smith (1989), "Public Support for Civil R-D in the UK: Limitations of Recent Policy Debate", Research Policy, Vol. 18, N², pp. 99-110.

${ }^{21}$ Quelques-uns de ces aspects sont examinés dans N.E. Terleckyj (1980), op.cit., pp. 55-61.

22 Voir les arguments avancés par J. Hansen (1986), op.cit., p. 8.

23 Afin d'obtenir des renseignements sur les innovations industrielles, Edwin Mansfield a réalisé une enquête auprès de quelque 70 grandes entreprises des États-Unis qui avaient exploité les résultats de recherches universitaires effectuées au cours des 15 dernières années. Il a estimé que les produits reposant sur des travaux universitaires récents représentaient 5 pour cent environ de la production industrielle des États-Unis. E. Mansfield (1988), “The Social Rate of Return From Academic Research", Rapport à la "Division of Policy Research and Analysis", National Science Foundation, Washington, pp. 23 + vii. Selon une enquête analogue menée au début des années 80 auprès d'industriels japonais, 60 pour cent de ceux-ci ont jugé inutilisables les résultats de la recherche fondamentale et appliquée réalisée dans les laboratoires universitaires tandis que 27 pour cent d'entre eux les ont trouvés utiles. S'agissant des résultats obtenus dans les laboratoires publics, près de 49 pour cent des industriels interrogés ont déclaré qu'ils n'étaient pas utiles contre 34 pour cent d'un avis contraire.

${ }^{24}$ Malecki, E. (1980), "Dimensions of R-D Location in the United States”, Research Policy, Vol. 9, №1, pp. 2-22.

25 Désignés, dans la version précédente du manuel, par le terme "innovation radicale de produit".

26 Désignés, dans la version précédente du manuel, par le terme "innovation progressive de produit".

27 Pour les États membres de l'Espace économique européen (EEE), l'entreprise est définie comme correspondant à "la plus petite combinaison d'unités légales qui constitue une unité organisationnelle de production de biens et de services jouissant d'une certaine autonomie de décision, notamment pour l'affectation de ses ressources courantes. Une entreprise exerce une ou plusieurs activités dans un ou plusieurs lieux" (Règlement (CEE) N696/93 du Conseil du 15 mars 1993, relatif aux unités statistiques d'observation et d'analyse du système productif dans la Communauté, J.O. $\mathrm{N}^{\circ} \mathrm{L} 76 / 5$, section III/A de l'annexe)

28 Voir CITI Rév.3, paragraphes 91 à 98.

29 "L'unité d'activité économique (UAE) regroupe au sein d'une entreprise l'ensemble des parties qui concourent à l'exercice d'une activité du niveau classe (quatre chiffres) de la nomenclature NACE Rév.1. Il s'agit d'une entité qui correspond à une ou plusieurs subdivisions opérationnelles de l'entreprise. L'entreprise doit disposer d'un système d'information permettant de fournir ou de calculer pour chaque UAE au moins la valeur de la production, des consommations intermédiaires, des frais de personnel, de l'excédent d'exploitation, ainsi que l'emploi et la formation brute de capital fixe." (Règlement (CEE) N696/93 du Conseil du 15 mars 1993 relatif aux unités statistiques d'observation et d'analyse du système productif dans la Communauté, J.O. $\mathrm{N}^{\circ} \mathrm{L} 76 / 8$, section III/D de l'annexe).

30 "L'unité locale correspond à une entreprise ou une partie d'entreprise (atelier, usine, magasin, bureau, mine, entrepôt) sise en un lieu topographiquement identifié. Dans ce lieu, ou à partir de ce lieu, sont exercées des activités économiques pour lesquelles, sauf exception, une ou plusieurs personnes travaillent (éventuellement, à temps partiel) pour le compte d'une même entreprise." (Règlement CEE Nº696/93 du Conseil du 15 mars 1993 relatif aux unités statistiques d'observation et d'analyse du système productif dans la Communauté, J.O. N ${ }^{\circ} \mathrm{L}$ 76/8, section III/F de l'annexe).

31 Pour un examen détaillé du problème de l'unité locale en tant qu'unité statistique dans les enquêtes sur l'innovation, voir Eurostat (1996), La dimension régionale de la $R-D$ et des statistiques relatives à l'innovation, notamment la partie B.

32 Voir CITI Rév.3, paragraphe 114 (ONU, 1990).

33 Voir CITI Rév.3, paragraphe 115 (ONU, 1990). 
34 Pour la définition, voir OCDE (1994), Méthode type proposée pour les enquêtes sur la recherche et le développement expérimental - Manuel de Frascati 1993, Paris, paragraphe 147.

35 OCDE (1994), Méthode type proposée pour les enquêtes sur la recherche et le développement expérimental Manuel de Frascati 1993, Paris, paragraphe 164.

36 Par exemple, au Canada, 40 pour cent des entreprises du secteur manufacturier sont innovantes tandis que 4 pour cent exécutent de la R-D et toutes les unités qui exécutent de la R-D ne sont pas innovantes dans la mesure où elles ne commercialisent pas leurs inventions.

37 Nations Unies (1990), Classification internationale type par industrie, de toutes les branches d'activité économique, Etudes statistiques, Série M, N 4, Rév. 3, New-York.

38 Commission européenne, Règlement (CEE) N $N^{\circ} 3037 / 90$ du Conseil du 9 octobre 1990, relatif à la Nomenclature statistique des activités économiques dans la Communauté européenne, Journal officiel des Communautés européennes, $\mathrm{N}^{\circ}$ L 293, Luxembourg 1990, modifié par le Règlement (CEE) № 761/93 de la Commission, Journal officiel des Communautés européennes, № L 83, Luxembourg 1993.

39 Eurostat, Nomenclature des unités territoriales statistiques, Luxembourg, dernière édition.

40 Il est difficile, sinon impossible, de définir quand un taux de non-réponse globale est réputé élevé ou faible. Toutefois, il est généralement admis que la comparabilité des résultats des enquêtes (sur l'innovation) est d'autant plus faible que le taux de non-réponse globale est élevé.

41 Une telle méthode a été appliquée avec succès lors de la série actuelle d'enquêtes sur l'innovation menée en Allemagne, aux Pays-Bas, en Irlande et au Danemark (CIS).

42 Les premières considérations méthodologiques peuvent être trouvées dans D. Archibugi, P. Cohendet, A. Kristensen, K.-A. Schäffer (1995), Evaluation de l'Enquête communautaire sur l'innovation (CIS) - Phase 1, Chapitre 6.4, Luxembourg.

43 Voir, par exemple, le Livre vert sur l'innovation, Bulletin de l'Union européenne, Supplément 5/95, Luxembourg, 1996.

44 L'ABS tenait particulièrement à mesurer l'innovation non technologique parce que son enquête sur la période 1993-1994 s'étendait au secteur des services. Parce qu'elle englobait les secteurs manufacturiers et des services, l'enquête a pu révéler l'ampleur de l'innovation non technologique dans l'économie australienne et, ce qui est important, elle a permis de comparer la fréquence de l'innovation technologique et celle de l'innovation non technologique. En résumé, l'enquête australienne a révélé que l'innovation non technologique était importante dans le secteur manufacturier, puisqu'elle a été constatée dans 24 pour cent des entreprises (contre 34 pour cent pour l'innovation technologique), mais que son importance relative était plus grande dans le secteur des services (14 pour cent des entreprises, contre 12 pour cent pour l'innovation technologique). Au total, l'innovation non technologique a été constatée dans 15 pour cent des firmes, et l'innovation technologique dans 13 pour cent. 